\title{
GeMS (Geologic Map Schema) - A Standard Format for the Digital Publication of Geologic Maps
}

Chapter 10 of

Section B, U.S. Geological Survey Standards, of

Book 11, Collection and Delineation of Spatial Data

Techniques and Methods $11-$-B10 
Cover. Geologic map of the western United States and surrounding areas, extracted from the "Geologic map of North America" (Reed and others, 2005; database from Garrity and Soller, 2009). Image downloaded from the National Geologic Map Database (https://ngmdb.usgs.gov/Prodesc/proddesc_86688.htm). 


\section{GeMS (Geologic Map Schema)-A Standard Format for the Digital Publication of Geologic Maps}

By the U.S. Geological Survey National Cooperative Geologic Mapping Program

Chapter 10 of

Section B, U.S. Geological Survey Standards, of

Book 11, Collection and Delineation of Spatial Data

Techniques and Methods 11-B10 


\title{
U.S. Department of the Interior DAVID BERNHARDT, Secretary
}

\section{U.S. Geological Survey James F. Reilly II, Director}

\author{
U.S. Geological Survey, Reston, Virginia: 2020
}

For more information on the USGS - the Federal source for science about the Earth, its natural and living resources, natural hazards, and the environment—visit https://www.usgs.gov or call 1-888-ASK-USGS (1-888-275-8747).

For an overview of USGS information products, including maps, imagery, and publications, visit https://store.usgs.gov.

Any use of trade, firm, or product names is for descriptive purposes only and does not imply endorsement by the U.S. Government.

Although this information product, for the most part, is in the public domain, it also may contain copyrighted materials as noted in the text. Permission to reproduce copyrighted items must be secured from the copyright owner.

Suggested citation:

U.S. Geological Survey National Cooperative Geologic Mapping Program, 2020, GeMS (Geologic Map Schema)—A standard format for the digital publication of geologic maps: U.S. Geological Survey Techniques and Methods, book 11, chap. B10, 74 p., https://doi.org/10.3133/tm11B10.

ISSN 2328-7055 (online) 


\section{Acknowledgments}

The GeMS database design is an outcome of years of research and collaboration by many scientists and GIS specialists, under the auspices of numerous projects and initiatives. The preparers of this document, Ralph A. Haugerud and David R. Soller (both USGS), especially thank Peter Lyttle (former Program Coordinator, National Cooperative Geologic Mapping Program) for his recommendation in 2008 that we undertake this work. We also thank our many colleagues who have given thoughtful comments and critiques of this design.

\section{Contents}

Introduction

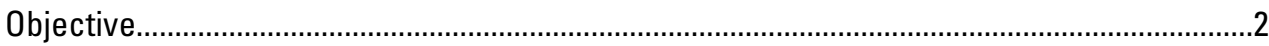

Lessons Learned in the Last Three Decades ..........................................................................

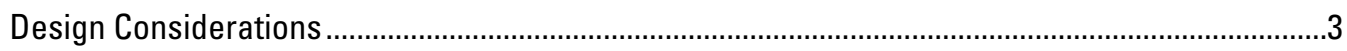

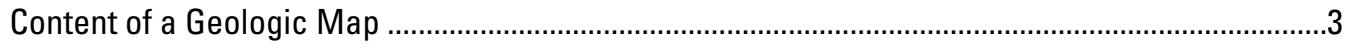

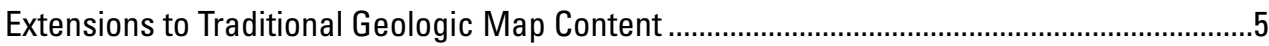

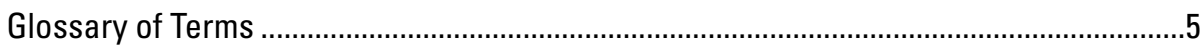

Classification of Geologic Materials ........................................................................

Feature-Level Metadata ..........................................................................................

Data Source (Provenance) ........................................................................................

Locational Confidence (Spatial Accuracy) ..............................................................

Scientific Confidence, Identity Confidence, and Existence Confidence ...................8

Orientation Confidence ..........................................................................................

Working with Multiple Feature Attributes .....................................................................

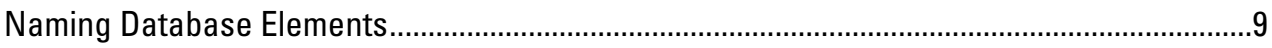

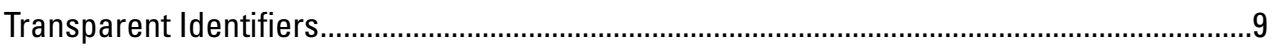

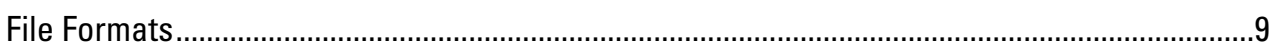

Required, As-Needed, and Optional Elements of a Digital Geologic Map Publication....................10

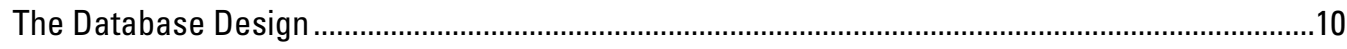

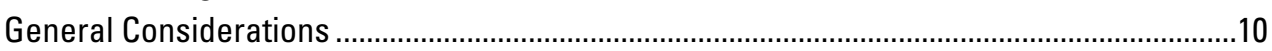

This Design Uses a Relational Database .......................................................................10

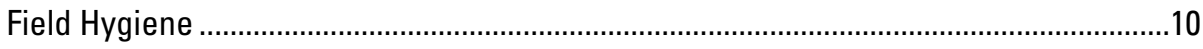

Type, MapUnit, Label, and Symbol Fields ....................................................................12

Polygons, Lines, and Topology—What Goes Where? ?..................................................16

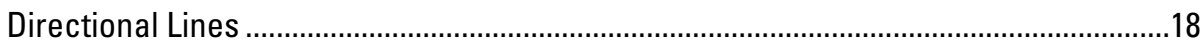

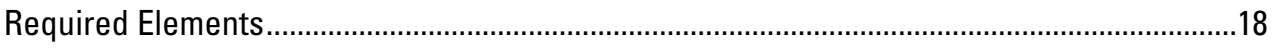

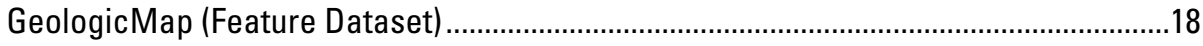

MapUnitPolys (Polygon Feature Class).............................................................18

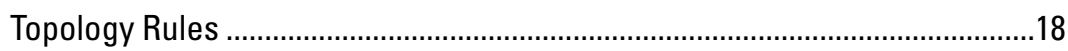

ContactsAndFaults (Line Feature Class) .......................................................18

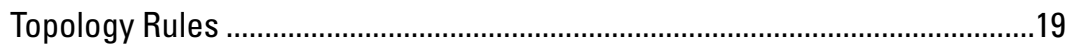

DescriptionOfMapUnits (Nonspatial Table)_......................................................................20

Notes on the Content of the Description Field .......................................................21 
Populating the DescriptionOfMapUnits Table......................................................24

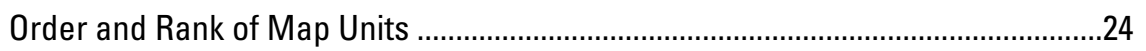

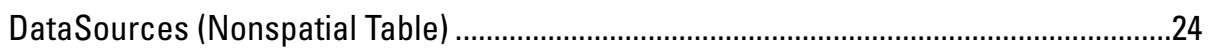

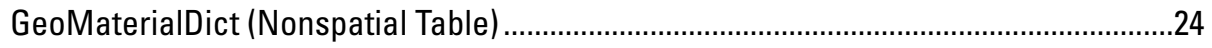

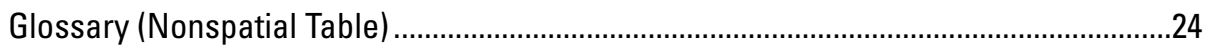

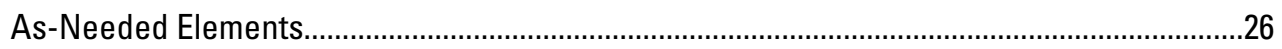

Guidelines for Naming and Designing Additional Feature Classes.................................26

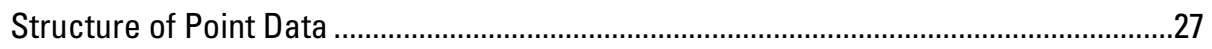

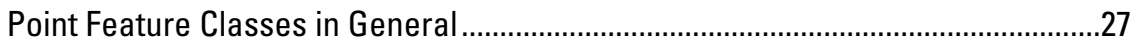

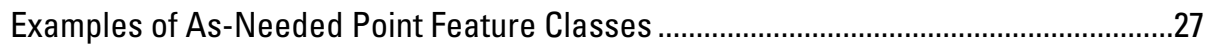

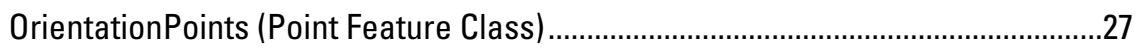

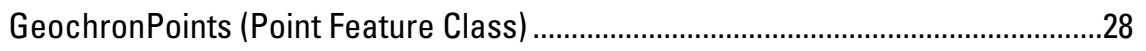

Stations (Point Feature Class) ............................................................................28

MapUnitPoints (Point Feature Class) …………......................................................32

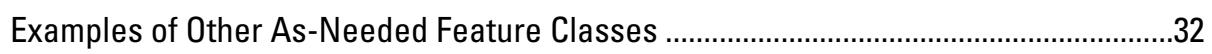

Geologic Lines (Line Feature Class) ......................................................................32

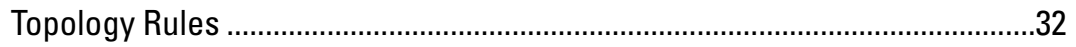

MapUnitLines (Line Feature Class) .........................................................................32

Topology Rules .........................................................................................32

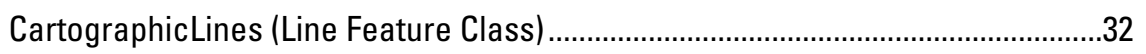

IsoValueLines (Line Feature Class) .........................................................................32

Overlay Polygons (the MapUnitOverlayPolys and OverlayPolys Polygon Feature

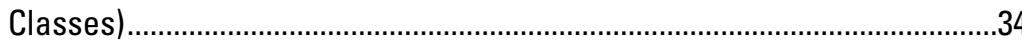

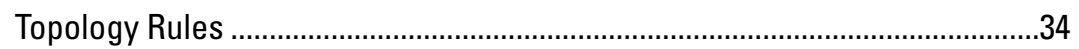

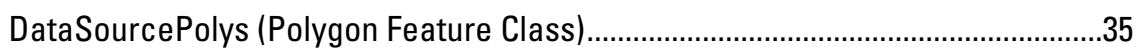

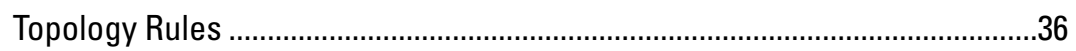

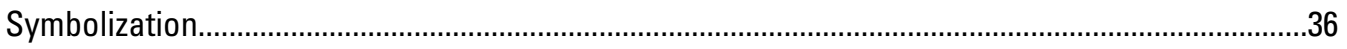

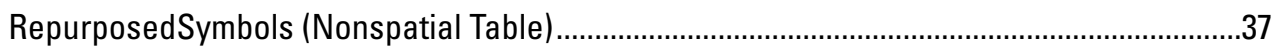

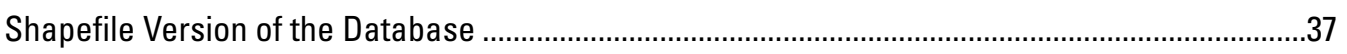

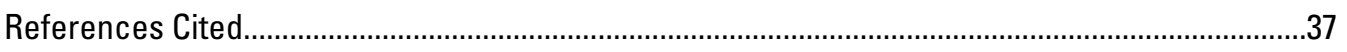

Appendix 1. Terms and Definitions for the GeoMaterial and GeoMaterialConfidence Fields ........40

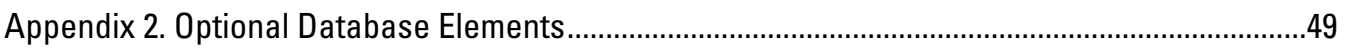

Appendix 3. Parsing a Description of Map Units (DMU) into the DescriptionOfMapUnits Table

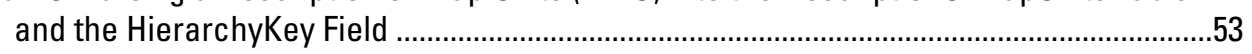

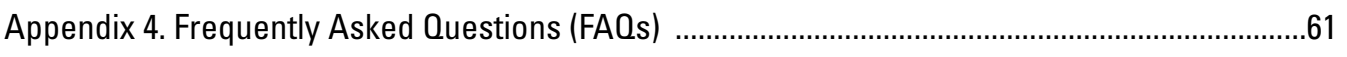

Appendix 5. Changes from NCGMP09 v.1.1 to GeMS .................................................................68 


\section{Figures}

1. ArcCatalog view of the contents of a GeMS-structured database, showing required, as-needed, and optional database elements...

2. Entity-relationship diagram for selected polygon feature classes and associated nonspatial tables, highlighting required and as-needed database elements. ... 13

3. Entity-relationship diagram for selected line feature classes and associated nonspatial tables, highlighting required and as-needed database elements.

4. Entity-relationship diagram for selected point feature classes and associated nonspatial tables, highlighting required and as-needed database elements.

3-1. Part of a modern Description of Map Units, excerpted from a geologic map published relatively recently.

3-2. Reproduction of part of a map-unit explanation from a somewhat older geologic map, showing an older style of map-unit description

3-3. Part of a modern Correlation of Map Units diagram, excerpted from a geologic map published relatively recently.

3-4. Part of an older map-unit explanation, excerpted from an older geologic map, showing an older, abbreviated style of map-unit description

3-5. Part of a relatively modern Correlation of Map Units diagram, excerpted from a geologic map published somewhat recently.

4-1. GeMS implementation of a stack-unit map that shows geologic materials to a depth of $20 \mathrm{ft}$, and the corresponding parts of tables MapUnitPolys, StackUnitsToMapUnits, and DescriptionOfMapUnits.

\section{Tables}

1. Example picklist of values for the LocationConfidenceMeters field..............................................

2. Example picklist of values for the IdentityConfidence field ......................................................... 8

3. Elements of a digital geologic map publication named "mapXYZ" ...........................................11

4. Required, as-needed, and optional contents of the zip archive (that is, the "mapXYZ-gdb.zip" file) that contains the database and other elements of the digital data package for a geologic map publication named "mapXYZ"

5. Required, as-needed, and optional contents of the resources folder that accompanies the database in a digital geologic map publication named "mapXYZ".

6. Examples of selected fields (and their values) in a DataSources table ..................................... 16

7. Examples of selected fields (and their values) in a DescriptionOfMapUnits table .................... 16

8. Examples of selected fields (and their values) in a ContactsAndFaults feature class.............. 16

9. Examples of selected fields (and their values) in an OrientationPoints feature class .............. 16

10. Examples of values in the Type, Symbol, and Label fields in a point feature class and the resulting map images

11. Examples of values in the MapUnit, IdentityConfidence, Symbol, and Label fields in the MapUnitPolys feature class and the resulting map images.

12. Fields in MapUnitPolys (a polygon feature class in the GeologicMap feature dataset) .......... 19

13. Fields in ContactsAndFaults (a line feature class in the GeologicMap feature dataset) ......... 20

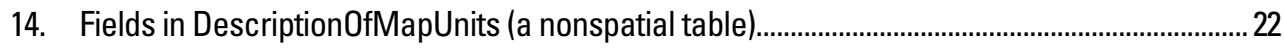

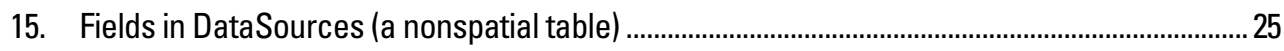

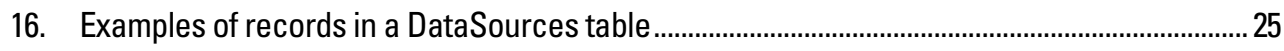

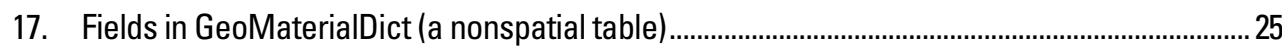




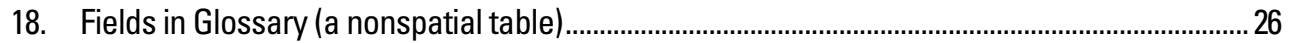

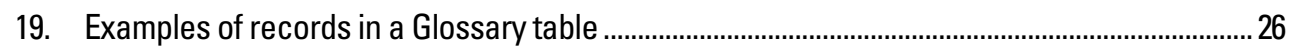

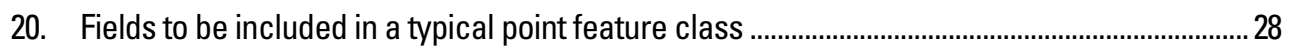

21. Recommended additional fields to be included in a sample-oriented point feature class...... 28

22. Fields in OrientationPoints (an as-needed point feature class in the GeologicMap feature dataset)

23. Fields in GeochronPoints (an as-needed point feature class in the GeologicMap feature dataset).

24. Fields in Stations (an as-needed point feature class in the GeologicMap feature dataset) ...31

25. Examples of additional fields that might be included in a Stations point feature class .............31

26. Fields in GeologicLines (an as-needed line feature class in the GeologicMap feature dataset)

27. Fields in CartographicLines (an as-needed line feature class in the GeologicMap feature dataset).

28. Fields in IsoValueLines (an as-needed line feature class in the GeologicMap feature dataset)

29. Fields in MapUnitOverlayPolys (an as-needed polygon feature class in the GeologicMap feature dataset)

30. Fields in OverlayPolys (an as-needed polygon feature class in the GeologicMap feature dataset)

31. Fields in DataSourcePolys (an as-needed polygon feature class in the GeologicMap feature dataset).

32. Fields in RepurposedSymbols (an as-needed nonspatial table)

1-1. Values (and their definitions) that populate the GeoMaterialConfidence field in a DescriptionOfMapUnits table

2-1. Fields in CMUMapUnitPolys (a polygon feature class in the CorrelationOfMapUnits feature dataset).

2-2. Fields in CMULines (a line feature class in the CorrelationOfMapUnits feature dataset).........50

2-3. Fields in MiscellaneousMapInformation (a nonspatial table) .................................................... 51

2-4. Fields in StandardLithology (a nonspatial table) .....................................................................

2-5. Examples of records in a StandardLithology table ............................................................. 52

3-1. Selected fields and values from a DescriptionOfMapUnits table for the Description of Map Units depicted in figure $3-1$

3-2. Selected fields and values from a DescriptionOfMapUnits table for the explanation depicted in figure 3-2

3-3. Selected fields and values from a DescriptionOfMapUnits table for the Correlation of Map Units diagram depicted in figure 3-3.

3-4. Selected fields and values from a DescriptionOfMapUnits table for the explanation depicted in figure $3-4$.

3-5. Selected fields and values from a DescriptionOfMapUnits table for the Correlation of Map Units diagram depicted in figure 3-5

4-1. Examples of selected fields (and their values) in LayerList (a nonspatial table), which organizes and describes datasets within a multidataset map.... 


\title{
GeMS (Geologic Map Schema)—A Standard Format for the Digital Publication of Geologic Maps
}

\author{
By the U.S. Geological Survey National Cooperative Geologic Mapping Program
}

\section{Introduction}

This report ${ }^{1}$ describes and defines GeMS, a new standardized database schema - that is, a database design - for the digital publication of geologic maps. It originally was intended for geologic mapping funded by the National Cooperative Geologic Mapping Program (NCGMP) of the U.S. Geological Survey (USGS), but its use can be extended to other programs and agencies as well. It is intended to bridge the gap between traditional geologic mapping and GIS communities at an operational level.

This schema was introduced at the Digital Mapping Techniques '09 meeting (May 2009) for evaluation as version 0.8 .2 , in order to solicit preliminary comments and testing; at that time, it was named "NCGMP09" to reflect its target audience and the date of its initial release. Subsequently, version 1.0 was released October 14, 2009, for presentation at the Geological Society of America's 2009 Annual Meeting. In the months that followed, more extensive evaluations were received, and the design evolved in response: version $1.1^{[2]}$ was published at the end of 2011 as an article in USGS Open-File Report 2010-1335 (U.S. Geological Survey National Cooperative Geologic Mapping Program [USGS NCGMP], 2010). Several more years of

${ }^{1}$ This report supersedes an article in USGS Open-File Report 2010-1335, "NCGMP09 — Draft Standard Format for Digital Publication of Geologic Maps, version 1.1" (U.S. Geological Survey National Cooperative Geologic Mapping Program, 2010).

${ }^{2}$ Version 1.1 of this schema was prepared by (listed in alphabetical order) R.A. Haugerud (USGS), S.M. Richard (Arizona Geological Survey; now at USGIN Foundation), D.R. Soller (USGS), and E.E. Thoms (USGS).

${ }^{3}$ The current document was prepared by Ralph A. Haugerud and David R. Soller (both USGS), and it reflects significant input by numerous members of the USGS-Association of American State Geologists-Digital Mapping Techniques community, specifically by the following members of the NCGMP09 Working Group (listed in alphabetical order), who should be considered as contributors: Jennifer Athey (Alaska Division of Geological \& Geophysical Surveys), Gregory Barker (New Hampshire Geological Survey), Seth Bassett (Florida Geological Survey), Jennifer Carrell (Illinois State Geological Survey), Lorie Coiner (Virginia Division of Geology and Mineral Resources), Mary DiGiacomo-Cohen (USGS), John Dunham (Kansas Geological Survey), Tracey Felger (USGS), Trish Gallagher (Alaska Division of Geological \& Geophysical Surveys), Jacqueline Hamilton (Minnesota Geological Survey), Jordan Hastings (University of California, Santa Barbara), Mike Hendricks (Alaska Division of Geological \& Geophysical experience, evaluation, and discussion have led to this current version, ${ }^{3}$ renamed as "GeMS," for Geologic Map $\boldsymbol{S c h e m a . ~}{ }^{4,5}$

GeMS provides for the encoding in digital form of the content contained in geologic maps published by the USGS and by state geological surveys. It stipulates that an Esri database format be used, not only to adhere to USGS policy ${ }^{6}$ but also because Esri's ArcGIS is the most commonly used GIS in the USGS, in state geological surveys, and in the larger geologic mapping community. Nevertheless, migration to a nonproprietary format is a worthy goal, and GeMS has been designed with this in mind.

Although GeMS is designed for a single-map database, it also is intended to provide a stepping stone toward the development of multiple-map databases, in particular the National Geologic Map Database (NGMDB). The Geologic Mapping Act of 1992, and its subsequent reauthorizations, mandate the creation of a national archive of standardized geologic map content. The NGMDB Project functions on behalf of the NCGMP as coordinator of database design and maintenance, in cooperation with the Association of American State Geologists. The database design contained herein will significantly promote that goal. All questions or comments about GeMS should be directed via email to gems@usgs.gov.

Surveys), Lina Ma (Oregon Department of Geology and Mineral Industries), Phil Miller (New Mexico Bureau of Geology \& Mineral Resources), Bethany Overfield (Kentucky Geological Survey), Jay Parrish (Pennsylvania Geological Survey), Kevin Russell (Indiana Geological \& Water Survey), Evan Thoms (USGS), Frederic Wilson (USGS), Jeremy Workman (USGS), and Mark Yacucci (Illinois State Geological Survey). GeMS will be maintained by the USGS National Geologic Map Database Project; all correspondence should be addressed to gems@ usgs.gov.

${ }^{4}$ NOTE: Earlier versions of this document and any updates, as well as further information on GeMS and resources such as an example database and tools, are available at the GeMS website (https://ngmdb.usgs.gov/Info/standards/GeMS/). The current document is formally published at https://doi.org/10.3133/tm11B10.

${ }^{5}$ Note that the name GeMS has been used before in a similar context (see Dohar, 2004). We are pleased to reinforce GeMS as a useful acronym, and we think there is little likelihood of confusion.

'USGS' general policy is stated in USGS Policy 6.1.3, supplemented by USGS policy document "Requirements for Digital Map Products" (May 24, 1999, available at https://ngmdb.usgs.gov/Info/standards/dataexch/USGSpolicy.html; see section 3, but please disregard reference to the Spatial Data Transfer Standard [SDTS], which is no longer applicable). 
In our years of work prior to developing NCGMP09 and GeMS, we recognized that one single database design cannot suit all purposes, and this realization has been underscored by our colleagues' evaluations of this design. In addition, we acknowledge that a database most suited to the needs of a field geologist likely will not address the content and cartographic requirements of a single-map database that would be published for use by geologists and nongeologists alike, nor will it meet the requirements of a multiple-map database that would be maintained in perpetuity by a mapping agency. We further recognize that, for any of these purposes, settling on one single database design may be contentious, in part owing to the varying requirements of other endeavors (for example, for field systems, for requirements imposed by local geology, or for particular hardware). Nevertheless, we have developed a design that ought to be generally useful for most implementations, while recognizing that many will not find it their first or best choice or that they may need to extend it to suit their purposes. Compromise in design, without sacrificing the flexibility necessary for science-driven data and information management, is the path we sought during development of the GeMS standard.

\section{Objective}

Geologic mappers, geologic mapping agencies, and geologic map users will all benefit from a standard database design for the digital representation of geologic maps. This document describes such a design for the representation of a single geologic map. The design is focused on the publication, transfer, and archiving of map data and less on the creation of map data, the visual representation of map data, or the compilation of data from many different map sources. With increased use of this design, we anticipate reductions in the cost of map preparation, production, and publication (including data compilation and synthesis, review, editing, cartography, prepress work, training, and tool development).

For the purposes of this design, a single-map database means a package of data (bearing in mind that many geologic map data are inherently interpretive) that pertains to a particular portrayal of the geology of a certain area (that is, the map extent), which is directly analogous to a traditional paper geologic map. The database is attributed to an author or authors who either have collected original data and developed the database and portrayal or have compiled data from existing sources and developed the portrayal.

We focus on the design of a single-map database for two reasons: (1) it is the issue that the geologic community understands best, and (2) it is the problem that we perceive is most in need of a solution. The construction and maintenance of an enterprise, multiple-map database raises several issues that we do not address here, including versioning, multiple-scale representations, vocabulary management, maintenance of the stratigraphic lexicon, and access control.

\section{Lessons Learned in the Last Three Decades}

Geologic map producers have been developing and using GIS representations of geologic maps for more than three decades. In the course of this effort, we have all learned valuable lessons, some of which are listed below.

Map data are most usefully stored, shared, and analyzed in a GIS.-Although paper maps can be represented digitally by scanning them and storing the resulting image files, this is only a very small step towards making the map and its constituent data more easily accessible. Similarly, maps that are simply vector-graphic files (for example, those produced in desktop publishing software such as Adobe Illustrator) generally do not contain real-world, spatially explicit locations, nor do they explicitly state their thematic content and, thus, are not easily shared nor used for subsequent analysis. The most useful digital map data have feature attributes that are stored explicitly in database tables and feature locations that are provided in a real-world spatial reference framework (for example, UTM10, NAD83).

The distinction between map data and their symbolization is important. - Geologic map data primarily consist of feature attributes in database tables (for example, line \#27 is an accurately located thrust fault; line \#28 is an approximately located contact; line \#29 is the shoreline of Lake Erie on August 27, 1978). Symbolization of these data via line symbols, point symbols, colored areas, and patterns results in a map portrayal on screen or on paper. Storing map data in a GIS - as opposed to its symbolization in a drawing program - facilitates machine-assisted analyses of the data, gives greater flexibility for alternate symbolization, and eases reuse of the data at different scales.

Maps in a GIS need to be accompanied by metadata for both the overall database and its individual features. - Early GIS practitioners, largely limited by both storage space and database architecture, created a substantial number of map databases in which key data fields were populated only by map-specific symbols that were not defined within the database (for example, a map unit was identified as "Ks" without specifying that "Ks" is shorthand for "Cretaceous sandstone"); however, this approach is inadequate to effectively communicate the geology of an area. Mappers and map users benefit from feature-level metadata that describe both data source and quality. Reasons for such metadata include (1) most geologic maps contain data from mixed sources and of variable quality, (2) map data need to be closely linked to their authorship because such data mostly are interpretations made by authors, and (3) mapping commonly requires significant support from a governmental agency, an academic institution, a professional society, or private industry, and such support needs to be recognized.

Real-world database designs reflect compromises between the intrinsic complexity of geologic map data, the needs of geologists and GIS practitioners who work with the design, the capabilities of GIS and database software, and the limitations of the underlying computer systems.-Database designs that do not make such compromises are not likely to be widely used. Even the names of various database elements (spatial featuresets, tables, and 
fields) must be carefully crafted to be understood by users who have different backgrounds, to facilitate the adaptation and reuse of software tools, and to promote the distribution, translation, and compilation of data.

Obtaining widespread community acceptance for a particular database schema and its associated vocabularies, which may extend beyond the precedents set by traditional mapping methods, is difficult. - Although this conservatism may be a good thing because our mapping traditions embody a great deal of hard-won wisdom, it is also unfortunate because these traditions incorporate prior compromises, necessitated by the limitations of nondigital formats, that are unnecessary in a digital world.

And finally, a perception persists among some geologists that the comprehension of their maps may be beyond the capabilities of the uninitiated and that, therefore, these maps cannot—and should not-be expected to be readily useable by members of the public.-This is unfortunate because it is the public who needs the maps (and who have paid for them). As noted by then-USGS Director John Wesley Powell, "maps [need to be] designed not so much for the specialist as for the people, who justly look to the official geologist for a classification, nomenclature, and system of convention so simple and expressive as to render his work immediately [understandable]" (Powell, 1888).

We have endeavored to honor these lessons when designing the GeMS database schema, and we hope that the schema will contribute to a better understanding, and the wider use, of geologic map data.

\section{Design Considerations}

Within GeMS, we have attempted to do the following:

- Encode the fundamental content of a single, traditional paper geologic map.

- Focus equally on both the digital storage and the digital transfer of map data.

- Facilitate editing of the map (for example, all lines that bound map-unit polygons are in the same layer to facilitate enforcement of topological constraints).

- Facilitate the interactive display and query of map data.

- Provide a foundation for the publication-quality visualization of the map data.

- Define the names and types of data (feature datasets, feature classes, nonspatial tables, ${ }^{7}$ fields, and attributes) for all constituent elements of the map, using names that have obvious meaning to geologists and GIS practitioners alike, both for the convenience of all users and as a springboard for development of software tools.
- Introduce standardized terminology and definitions to address the perception that traditional geologic maps do not meet the public's (nor the scientist's) need for consistently named and defined earth materials.

- Preserve and facilitate the analysis of map-feature topology.

- Normalize map data for robustness and compactness, but not to the extent that user comprehension is reduced.

- Allow the descriptions of map features to be queried with as much (or as little) granularity or detail as desired.

- Facilitate the reuse of map data in later compilations by including feature-level metadata.

- Accommodate both Esri and open file formats for flexibility, interoperability, and data longevity.

\section{Content of a Geologic Map}

Geologic maps are rich in semantic content, fundamental aspects of which must be preserved in a database for it to be useful. Such fundamental content appears in MAGENTA ALL CAPS bold type in the outline below. Items in BLACK ALL CAPS bold type are secondary or supporting content. Digital forms for both primary and secondary content are further discussed below where the GeMS schema is described in detail. Items in black italic bold type are secondary or supporting content for which a digital form is not specified herein.

1. MAP GRAPHIC - Consists of some or all of the following:

A. Base map - Graphical elements that provide spatial and cultural context for map data at a certain scale (for example, shaded relief, topographic and (or) bathymetric contours, drainage features, township and range grid, transportation network, cultural features, place names); although not required, inclusion of a base map is strongly recommended.

B. MAP-UNIT POLYGONS-Polygons that cover the map area with no gaps or overlaps; may include areas of open water, permanent snowfields and glaciers, and unmapped areas.

C. CONTACTS AND FAULTS - Lines that, with a few exceptions, bound and separate map-unit polygons.

D. Other elements that are present as needed to record significant map content:

I. ORIENTATION DATA-Point features that record measurements of the orientation of rock fabrics (for example, bedding, foliation, paleocurrents, magnetic anisotropy). 
II. OVERLAY POLYGONS-Areal features that overlie, underlie, are within, or cut across mapunit polygons (for example, shear zones, alteration zones, areas of artificial fill, surface projections of mined-out areas); need not conform to topological rules that constrain map-unit polygons; and are commonly represented by patterned overlays whose boundaries are not drawn.

III. MAP-UNIT LINES AND POINTS—Line or point features that represent map units that are too small to show at scale of map graphic.

IV. MISCELLANEOUS LINES-Line features not categorized above (for example, dikes and sills, marker beds, traces of fold hinges, facies boundaries, structure contours, isograds, crosssection lines).

V. MISCELLANEOUS POINT DATA-Point features that record, for example, sample localities for geochronologic and chemical analyses, fossil localities, prospect locations, and displacement (fault-slip) measurements.

2. CROSS SECTIONS, as appropriate-Each cross section has elements that are analogous to those of the map (listed above), except that the base map is replaced by a topographic profile.

3. CORRELATION OF MAP UNITS (CMU) - Diagram that depicts the ages and relations of (and among) map units; may include headings and grouping brackets; sometimes shows nonconformities or additional data such as radiometric ages; is not intended to be a true stratigraphic section or column; usually does not include areas of open water, permanent snowfields and glaciers, or unmapped areas.

4. SYMBOLIZATION for all of the above, including the following:

\section{A. FILLS (COLORS AND OPTIONAL PATTERNS)} for map-unit polygons.

B. FILLS (PATTERNS) for overlay polygons.

C. LINE SYMBOLS AND (OR) POINT SYMBOLS for map-unit areas too small or narrow to show as polygons at map scale.

D. Text labels (or, in some cases, labels and leaders) for some (but not necessarily all) polygons.

E. LINE SYMBOLS HAVING VARIOUS COLORS, WEIGHTS, AND PATTERNS (DASHES, DOTS)Some line symbols contain repeated ornaments (for example, thrust teeth, queries), and some have single (nonrepeating) ornaments.
F. POINT SYMBOLS-Note that not all points in the database need be symbolized.

G. FONT FILES - If needed for point symbols or line-symbol ornaments.

H. Text labels (or, in some cases, labels and leaders) for some lines and groups of lines and for some points (for example, fault names, well identifiers).

5. DESCRIPTION OF MAP UNITS (DMU)—Contains labels, names, ages, and descriptions for each map unit shown on the map or in a cross section:

- A DMU contained on a map sheet will show, in addition to the map-unit label, the area-fill symbol (color and sometimes a pattern) used to depict the unit on the map.

- In some map reports, the DMU is not printed on the map sheet but, instead, is contained in an accompanying pamphlet; in these DMUs, typically only the map-unit label is shown, without color or pattern.

- DMUs can be strongly hierarchical, using headings (for example, "Unconsolidated Deposits") and paragraph styles (font size and style, indentation) to denote each unit's position in the hierarchy.

- DMUs may include bracketed headnotes (for example, "[Modified from Booth and others (2009)]").

- DMUs sometimes contain units that are not shown on the map or cross section (for example, a formation that is mapped entirely as its constituent subunits); such units will lack a map-unit label and an area-fill color or pattern in the DMU (because they are not depicted on the map), but, in order to fully communicate stratigraphic relations, their names and ages are listed in the DMU and their positions in the hierarchy are indicated.

- DMUs typically do not describe areas of open water, permanent snowfields and glaciers, unmapped areas, and certain geologic overlays.

6. List of Map Units ( $\boldsymbol{L M U})$, if applicable-A distillation of the DMU, placed on the map sheet as a key to help identify map units when the DMU is contained in an accompanying pamphlet; an LMU contains the same map-unit labels, names, and ages as does the DMU but not the full map-unit descriptions; an LMU also uses the same hierarchical elements (headings, type styles, indentations, and so on) as the DMU.

7. EXPLANATION OF MAP SYMIBOLS - Contains descriptions of line symbols, point symbols, and overlay patterns but (typically) not symbols for base-map features; map-symbol explanations can be hierarchical.

8. MISCELLANEOUS MAP-COLLAR INFORMATION_Consists of information such as report title, 
author(s), date of publication, publisher, series name and series number, map scale, various credit notes (for example, "mapped by", "edited by", "GIS database by", "cartographic production by"), and base credit note (for example, specification of spatial reference framework or projection, source[s] and scale[s] of base map); content may vary from agency to agency, from one mapper to another, and from one map to another.

\section{Discussion.}

\section{References Cited.}

11. Figures(s), if applicable.

12. Tables(s), if applicable.

13. Additional map(s), if applicable - For example, sources of map data, distribution of facies in the Cambrian.

It is worth noting that, in general, populating the database by assigning values to feature attribute fields is the responsibility of the database creator. In some cases, this may require manual entry of individual attribute values for each feature. However, in many cases, such assignments can be made in bulk or can be scripted, as noted below (see, for example, the scripts GeMS_SetSymbols Arc10.py and GeMS_SetPlotAtScales_Arc10.py; both of these, or their replacements, are available at https://github.com/usgs/ GeMS_Tools).

\section{Extensions to Traditional Geologic Map Content}

GeMS includes several extensions to traditional geologic map content. The following three are required: (1) a glossary of terms, (2) a simple classification of map-unit materials, and (3) certain feature-level metadata. Optional extensions may include supplemental standardized lithologic descriptions of map units and a simple table of miscellaneous map information.

\section{Glossary of Terms}

Many published digital geologic map databases, and many paper geologic maps as well, provide few, if any, definitions for the technical terms used to name and describe map features. Some producers of geologic map databases have remedied this by providing formal Federal Geographic Data Committee (FGDC) metadata, which can contain detailed entity and attribute descriptions that encapsulate definitions (and definition sources) for these terms. Unfortunately, such metadata can be difficult to access and nearly impossible to relate automatically to the relevant features in a database.

GeMS implements a Glossary table that, for certain database fields, lists the terms that populate these fields, along with their definitions and the sources for these definitions. Terminology used in these database fields must be defined in this Glossary table. Contents of the Glossary table may be accessed by means of a database join (or relate) that is based on the term itself.
Formal metadata for a feature class or table can then reference the Glossary table for definitions and definition sources; therefore, relisting these definitions within detailed entity and attribute metadata is not necessary. Terms used only in the Description field of a DescriptionOfMapUnits table need not be defined.

If populating the Glossary table seems excessively laborious, consider that, once terms are defined in a Glossary table, they are readily available for display within the map, either on screen or in print. Furthermore, they can be easily searched for and extracted for use in other publications. In most cases, definitions in the Glossary table can be copied directly (or paraphrased) from standard reference sources (for example, American Geosciences Institute's [AGI's] "Glossary of Geology (5th ed., revised)"; Neuendorf and others, 2011), with appropriate attribution, or from preexisting GeMS Glossary tables, with minor adjustments or amendments. Although building Glossary tables for the first few maps produced by a workgroup will be a significant effort, subsequent Glossary tables ought to become much easier to develop as content from previous Glossary tables is reused.

\section{Classification of Geologic Materials}

A DMU conveys essential information about each map unit and is a cornerstone of the GeMS design. However, descriptions in a DMU can vary greatly in their content and format, and they commonly use specialized terminology that may be unfamiliar to the nongeologist. In addition, terminology may be used inconsistently from map to map (usually for valid reasons). Over time, many attempts have been made to organize and standardize descriptions of geologic materials, with the goals of improving our abilities to make regional compilations and to more effectively convey geologic information to the public. Of necessity, such efforts are compromises that only partly describe the nearly infinite variety of map-unit ages, compositions, textures, modes of genesis, and appearances.

In 2004, the North American Geologic Map Data Model Steering Committee (see https://ngmdb.usgs.gov/www-nadm/) sponsored by the USGS, the Association of American State Geologists, and the Geological Survey of Canada - defined a general, conceptual data model for geologic maps, as well as a science language for describing various characteristics of earth materials. Their summary report on science language (North American Geologic Map Data Model Steering Committee Science Language Technical Team, 2004) presented classifications that have since been evaluated and adapted for many purposes; for example, the IUGS-CGI ${ }^{8}$ Geoscience Concept Definitions Working Group incorporated that work into a limited set of lithology categories ("SimpleLithology") for use in GeoSciML" interchange documents (see http://resource.geosciml.org/def/voc/).

${ }^{8}$ IUGS, International Union of Geological Sciences; CGI, Commission for the Management and Application of Geoscience Information.

${ }^{9} \mathrm{GeoSciML}$ is an XML-based data transfer standard for the exchange of digital geoscientific information. 
A similar list of terms ("StandardLithology") accompanied the initial release of NCGMP09 (version 1.0, October 2009). As with SimpleLithology, the StandardLithology list of terms was designed to be used with companion lists of proportion terms or values to encode the relative amounts of potentially numerous lithologies that could be found in each map unit. This approach encouraged multiple lithology entries for a map unit, thereby allowing detailed descriptions of map units. However, the level of effort required to populate StandardLithology was judged by many reviewers of version 1.0 to be too high. Therefore, this approach was abandoned for version 1.1 of NCGMP09 (and for GeMS).

Nevertheless, we remain convinced that standardized terminologies are beneficial, largely because of their potential to do the following:

- Allow more uniform portrayal of rock and sediment types across multiple maps.

- Facilitate queries for the presence of a particular rock type. For example, by using a hierarchical classification, both the queried rock type and its related rock types can be found. For example, if "lava flows" is queried, then both "felsic lava flows" and "igneous rock" may also be returned.

Bearing in mind the importance of providing the public a simple, systematic view of the Nation's geology, NCGMP09 v.1.1 included, and GeMS retains, a simplified classification of earth materials that is based on general lithologic and genetic character. This classification (called GeneralLithology in NCGMP09 but renamed GeoMaterial in GeMS) applies a single term to each map unit, providing information that a nonexpert could quickly use to identify map units that contain similar materials. Although GeoMaterial is a required field in GeMS, its content is not intended to be a substitute for the more detailed and precise lithologic terminology that would be included in a DMU or in a more detailed and specialized controlled-term list; rather, its purpose is as stated briefly above (and also as discussed in detail in Soller, 2009).

The GeoMaterial classification, which has been developed empirically, is based on commonly occurring geologic materials. It serves to organize the many and varied geologic material terms found in the source maps, which represent a wide range of geologic conditions across the Nation, in order to provide a consistent means of displaying map-unit materials. It was initially developed for the NGMDB Data Portal, a prototype site ( $c a .2008$ ) intended to raise discussion with NGMDB partners in the state geological surveys regarding how to provide the public with an integrated view of regional-scale geologic maps, with links to the source-map information. Documentation of the original term list, including the rationale, was provided in Soller (2009). The GeneralLithology classification in version 1.1 of NCGMP09 was slightly modified from the original, and it has been further modified (slightly) on the basis of six years of evaluation and test implementation by the state geological surveys and USGS. For inclusion in this version (version 2, GeMS), it has been renamed GeoMaterial. Note that, given the lengthy period of evaluation, as well as the inherent challenges in modifying a controlled-term list (for example, updating previously published databases to a revised classification), we do not anticipate modifications to this list in the near future.
For some purposes, a single, standard earth-materials classification may not adequately address the geology of a given region in sufficient detail. Therefore, scientists may wish to attach a terminology to their research databases that is more detailed and structured than what is stipulated in GeMS. A more structured controlled-term list, for example, might be desired in order to query a database for minor lithologies within a map unit that are not adequately indicated, either by the map-unit name, its GeoMaterial term, or its Description in the DescriptionOfMapUnits table. In such cases, evaluation of the salary and programming costs versus the research and societal benefits of including supplemental data tables and vocabularies may motivate a mapping project to extend the GeMS schema; if so, we advocate using either an optional table of the geologist's own design or the optional StandardLithology table (described in appendix 2).

\section{Feature-Level Metadata}

All features in a geologic map database should be accompanied by an explicit record of the data source. Many features should also be accompanied by explicit statements of scientific confidence- for example, how confident is the author that a feature exists? Or that it is correctly identified? How confidently are feature attributes known? We recognize that these are challenging questions to which the field geologist may not be comfortable providing an answer, except in the most general sense. But we also recognize that geologic information commonly is used in a GIS in conjunction with other types of information (for example, cadastral surveys, road networks, pipelines) and that terms such as "accurately located" have a markedly different meaning for a pipeline or property line than for a geologic contact. Thus, in order to provide a general indication of the scientific (existence and identity) confidence and locational accuracy of geologic-map features, GeMS implements per-feature descriptions of scientific confidence and locational accuracy. For more discussion on this topic, please see Section 4 in the introductory text of the "FGDC Digital Cartographic Standard for Geologic Map Symbolization" (Federal Geographic Data Committee [FGDC], 2006, available online at https://ngmdb.usgs.gov/fgdc_gds/geolsymstd.php; see also U.S. Geological Survey, 2006). The following sections discuss the feature-level metadata required in GeMS.

\section{Data Source (Provenance)}

Typically, a single map database will have very few datasource records because many features will have identical sources. For a database composed entirely of new mapping, a single data source may be cited ("this report"). Some data elements will have compound sources: for example, geochemical analysis of a rock sample will typically have one source for the map location and stratigraphic provenance of the sample (that is, the field geologist's reported data) and another source for the chemical analysis (that is, the geochemist's reported data). In such cases, having multiple source fields in the data table would be appropriate (for example, LocationSourceID and AnalysisSourceID). 


\section{Locational Confidence (Spatial Accuracy)}

Reported locations of geologic features and observation points commonly are uncertain for a number of reasons. For example, (1) there may be error in locating observation points because of global positioning system (GPS) errors or an imprecise base map, (2) positions of subtle features may be poorly known relative to well-located observation points, or (3) the locations of features may be known only by inference from locations of other features or observations. This uncertainty could be expressed as uncertainty in absolute location (geodetic accuracy); however, because uncertainty in absolute location often is unknown (especially with legacy data), because most users locate geologic features in relation to a base map, and because most spatial analyses of geologic map data are in relation to the base map or to other data in the same database, we've chosen to focus on uncertainty of location relative to the positions of other features in the database (for example, "How well located is this contact with respect to surrounding lithologic and strike-and-dip observations?"). With a sufficiently large database, this is equivalent to uncertainty in location relative to the base map.

We define locational confidence (contained in the database field LocationConfidenceMeters) as the combination of the positioning error of a known point relative to the base map ("How precisely do I know where I am?") and the uncertainty in location of a geologic feature relative to that known point ("How precisely, relative to where I am, can I place this contact?"). For a wellexposed, sharp contact, the second factor would be zero, and the locational confidence becomes equivalent to the positioning error.

This usage differs from that advocated by Section 4.2 in the introductory text of the FGDC cartographic standard (FGDC, 2006), which suggested that spatial accuracy be expressed using the following three attributes: (1) locatability (values of "observable", "inferred", or "concealed"); (2) zone of confidence (value of a distance [for example, a value equivalent to one-twenty-fifth of an inch at map scale], which may or may not be the same for all parts of a map); and (3) positioning (values of "within zone of confidence" or "may not be within zone of confidence"). We have departed from the recommendation in the FGDC cartographic standard in order to create databases that are simpler to understand and are less dependent upon visualization scale. In addition, we believe our approach is more informative because the FGDC cartographic standard does not include guidance for quantitatively recording how precisely a feature is located if it is not positioned within the zone of confidence.

For point features, LocationConfidenceMeters should be reported as the estimated radius (in meters) of the circle of uncertainty around the point location. For line features, it should be the half-width of the zone within which a line is asserted to be located. Values of LocationConfidenceMeters are recorded as floatingpoint numbers because they are real, measurable quantities, not because they are precisely known. Table 1 shows an example picklist of values for the LocationConfidenceMeters field that may provide insight into how this field may be populated. Note that the values in this picklist (table 1) are merely suggestions; use of other values for LocationConfidenceMeters certainly is acceptable. In situations where locational confidence changes along the length of a line, it may be best to split the line and assign different values of LocationConfidenceMeters to the different line segments.

Values of LocationConfidenceMeters can be visualized with semitransparent, proportional-width symbols (similar to buffers in GIS jargon) in which the half-widths of the semitransparent symbols are equal to the LocationConfidenceMeters values assigned to the features. Such visualizations are powerful tools for evaluating the appropriateness of LocationConfidenceMeters values as a map is being prepared.

When new geologic map databases are created, we expect that geologists will assign values of LocationConfidenceMeters to individual features or groups of features on the basis of their knowledge of mapping procedures, field conditions, and the nature of specific features. Even with, for example, a factor-of-two uncertainty, such values assigned by the original mapper or author are preferable to a null value. When transcribing legacy maps, an experienced field geologist will commonly be able to estimate useful values for LocationConfidenceMeters. In rare cases, such estimation may not be practical, and, in such cases, traditional qualitative descriptors of line accuracy such as "contact, approximate" may be placed in the Type field; the meanings of such

Table 1. Example picklist of values for the LocationConfidenceMeters field.

[Abbreviations: DEMs, digital elevation models; GPS, global positioning system; m, meter(s); NAIP, National Agriculture Imagery Program]

\begin{tabular}{cc}
$\begin{array}{c}\text { Example } \\
\text { value }(\mathbf{m})\end{array}$ & Comments \\
\hline 5 & $\begin{array}{c}\text { Appropriate for well-defined features located in the field by clear-sky GPS, by inspection of high-resolution topography (for } \\
\text { example, 1- or 2-m-resolution lidar DEMs), or by inspection of large-scale, well-rectified digital orthophotographs (for } \\
\text { example, NAIP images) }\end{array}$ \\
& $\begin{array}{c}\text { Reasonable for locations established by inspection of 1:24,000-scale maps, or for "accurately located" features digitized from } \\
1: 24,000 \text {-scale paper source maps }\end{array}$ \\
& $\begin{array}{c}\text { May be appropriate for some "approximately located" lines on 1:24,000-scale maps; other "approximately located" lines on } \\
\text { the same map may have values of } 100 \text { m or more }\end{array}$ \\
& Appropriate for "accurately located" features digitized from 1:100,000-scale paper source maps \\
\hline 50 & $\begin{array}{c}\text { Appropriate for "accurately located" features digitized from 1:250,000-scale paper source maps, or when a geologist, working } \\
\text { at 1:24,000 scale, says, "My confidence in locating this feature is exceptionally low" }\end{array}$ \\
\hline
\end{tabular}


qualitative descriptors must be defined in the Glossary table. A null value (for example, value $=-9$; see discussion below) may then be assigned to LocationConfidenceMeters; note that this a numeric value, not a text string.

For certain types of lines (for example, most map boundaries), positions are calculated or assigned, not observed. For these lines there is no positional uncertainty, and LocationConfidenceMeters should be assigned a value of 0.0 .

\section{Scientific Confidence, Identity Confidence, and Existence Confidence}

According to the FGDC cartographic standard (FGDC, 2006), scientific confidence may have either a single dimension or multiple dimensions. For a map-unit area, scientific confidence will have one dimension (that is, confidence that the map unit is correctly identified). In the case of faults, contacts, and other feature traces, the situation is more complex. For example, uncertainty may arise as to whether a boundary between two units is a contact or fault, or what kind of fault is mapped; in both cases, this uncertainty would be specified by an identity confidence value. In some cases, however, one may suspect (but not be certain) that a fault is present. Similarly, one may map features such as fold-hinge surface traces, dikes, and marker beds where their existence is suspected but not certain. These uncertainties would be specified by an existence confidence value such as "questionable". Note that contacts rarely are mapped where their existence is uncertain; if different map units are identified, a boundary of some sort must exist between them, and so the identity of that boundary may be questionable, but not its existence.

GeMS includes ExistenceConfidence and IdentityConfidence fields for line feature classes and an IdentityConfidence field for polygon and point observation features. We discussed at length whether to combine these confidence concepts into a single ScientificConfidence field in the database, perhaps with four or six values that would allow for various combinations of existence and identity confidence, but we decided that it makes more sense to separate them, as is specified in the FGDC cartographic standard. In many situations, default values for an entire map area would be appropriate, as is noted elsewhere in this report; in other situations, perhaps tools to efficiently assign varying confidence values can be developed by the GeMS user community. We expect that symbolization will, in many cases, be assigned on the basis of the appropriate confidence terms and feature type.

For most databases, all values of ExistenceConfidence and IdentityConfidence likely will be either "certain" or "questionable" (see table 2), although GeMS allows values and definitions other than these. Values of ExistenceConfidence and IdentityConfidence must be defined in the Glossary table. For some digital transcriptions of legacy or paper geologic maps, it may not be possible for the transcriber to assign values of ExistenceConfidence or IdentityConfidence; in these cases, the value of "unspecified" should be used. Note, however, that if a reviewer during the review process encounters a value of "unspecified" in a database of new mapping, its use should be questioned.
Table 2. Example picklist of values for the IdentityConfidence field.

[Definitions modified from FGDC cartographic standard (Federal Geographic Data Committee, 2006), p. 16-17, A-iii]

\begin{tabular}{|c|c|}
\hline Value & Definition \\
\hline certain & $\begin{array}{l}\text { Identity of a feature can be determined using } \\
\text { relevant observations and scientific judgment; } \\
\text { therefore, one can be reasonably confident in } \\
\text { the credibility of this interpretation }\end{array}$ \\
\hline questionable & $\begin{array}{l}\text { Identity of a feature cannot be determined using } \\
\text { relevant observations and scientific judgment; } \\
\text { therefore, one cannot be reasonably confident } \\
\text { in the credibility of this interpretation. For } \\
\text { example, IdentityConfidence = "questionable" } \\
\text { would be appropriate when a geologist reasons, } \\
\text { "I can see some kind of planar feature that } \\
\text { separates map units in this outcrop, but I } \\
\text { cannot be certain if it is a contact or a fault" }\end{array}$ \\
\hline
\end{tabular}

\section{Orientation Confidence}

For orientation measurements (bedding, foliation, lineation, joints, etc.), it is useful to describe how accurately the orientation has been measured. For linear features (for example, fold axes, lineations), this error is usefully expressed as the radius of the error circle, similar to the $\mathrm{alpha}_{95}$ value often reported for paleomagnetic directions. For planar features (for example, bedding, foliation), the error is that of the pole to the plane. The OrientationPoints feature class includes an OrientationConfidenceDegrees field to record this uncertainty. Values of OrientationConfidenceDegrees are recorded as floating-point numbers because they are real, measurable quantities, not because they are precisely known.

\section{Working with Multiple Feature Attributes}

Some users of this schema will have had experience using databases in which features have a single attribute field (for example, LTYPE) and a single attribute (for example, "contact, inferred, queried") that both defines the feature type and describes its locational and (or) scientific confidence. For these users, the use of multiple feature attributes (for example, Type and LocationConfidenceMeters for line features) prescribed herein for feature-level metadata may appear to require a significant increase in the amount of work needed to create a database. Although this document is not a vehicle for workflow suggestions, we note that simple modifications of existing workflows can greatly ease the workload of assigning multiple attributes to features. For example, features could be digitized and attributed with a single interim attribute value that could later be used to drive a script that assigns multiple attributes (for example, GeMS_AttributeByKeyValues_Arc10.py or its replacement, available at https://github.com/usgs/GeMS_Tools). In addition, feature templates in ArcGIS could be used to create features that have clusters of common attribute values. A workflow that tracks the genesis (that is, the initial creation) of 
features, perhaps by means of the DataSource attribute, may be useful for the bulk assignment of confidence attributes.

In some cases, default data source, scientific confidence, and locational accuracy values for an entire map area are appropriate. Although default values may seem meaningless, changes in default values from map to adjacent maps, as well as between geologic and other GIS layers (for example, pipeline routes), are likely to be informative to map users. As software tools evolve, we anticipate changing workflows that produce more detailed metadata.

\section{Naming Database Elements}

Standardized names for database feature datasets, feature classes, nonspatial tables, and fields that clearly convey their meaning are critical to a functional database design. Field names in GeMS have been chosen according to the following rules:

- Field names convey content to the geoscientist, to the GIS practitioner, and to the public.

- Long field names are acceptable and informative.

- Field names are easy to code and to calculate.

- Field names reflect data type.

- Field names use uniform concatenation protocol (in this case, PascalCase; that is, the first letter of each word is upper case).

- Field names do not exploit case sensitivity (note that case needs to be conserved because some languages and operating systems distinguish between "ThisName" and "thisName").

- Field names do not contain spaces or special characters.

- Names that contain “_ID” (for example, TableName_ID, FeatureClassName_ID) are reserved for primary keys, ${ }^{10}$ which are maintained by the database creator (not the GIS software) and are used mostly to relate attributes stored in nonspatial tables to spatial features, as well as (optionally) to relate spatial features to additional, feature-specific attributes stored in other tables.

- Names that contain "SourceID" (for example, DataSourceID, LocationSourceID) are reserved for foreign keys ${ }^{11}$ to the DataSources table.

Names for feature datasets, feature classes, and nonspatial tables follow similar rules. Note that we have chosen to not encode the publication identity (that is, the map name and series number)

\footnotetext{
${ }^{10} \mathrm{~A}$ primary key is a unique identifier for each record in a table within a relational database. It may be used to join (or relate) two tables. A table can have only one primary key, and the key field cannot contain null values.

${ }^{11} \mathrm{~A}$ foreign key is a key used to link two tables together in a relational database. It is a field (or collection of fields) in one table that refers to the primary key in another table.
}

in such names. Although doing so would simplify the joint display of multiple publications in an ArcMap project (because each layer name would automatically include the publication identifier for the feature class), our choice to use the same names in each delivery database keeps the naming scheme simple and facilitates the coding and sharing of tools to manipulate databases. We note that layer names in an ArcMap project can be easily changed to reflect the source database if desired.

\section{Transparent Identifiers}

In the database, the identifiers for map units, line types, and point feature types should be transparent; in other words, they should all have obvious, easily understood meanings. In the MapUnitPolys feature class, the map-unit identifier (MapUnit) is a key to the DescriptionOfMapUnits table from various other tables, and it should correspond to, but may not be identical to, the label for that unit on the map (for example, Qal; see discussion of Labels below). However, some DMUs contain map units that are not symbolized either on the map, in the CMU, or in a cross section (for example, a formation that is mapped entirely as its subunits); these units will have null map-unit identifiers in the DescriptionOfMapUnits table, as will DMU headings and headnotes.

The type identifiers for lines and points are references to terms in the Glossary table, and, for this and other reasons, we recommend that these simply be the geologic term for the type of line or point feature represented. This is in contrast to a common practice that dictates that identifiers used as foreign keys in a database consist of numbers or text strings having no inherent or obvious meaning to users; these commonly are referred to as opaque identifiers. Although opaque identifiers may be more robust as foreign keys in a database, we assert that, to facilitate comprehension and use of information from a database, the use of transparent (that is, easily understood) identifiers is preferable. Note, however, that GeMS does not prohibit the use of opaque identifiers, particularly for primary key values (for example, TableName_ID).

\section{File Formats}

In principle, we encourage the use of open file formats because (1) open formats facilitate the writing and redistribution of third-party code; (2) open formats reduce the risk of losing data when the format becomes obsolete and unreadable (when open formats are superseded, documentation for them is likely to remain available); and (3) open formats are more likely to change in a measured fashion than are proprietary formats. Many in the geologic mapping community are still coping with the costs of the relatively rapid transitions from Arc/Info coverages to shapefiles to ArcGIS personal, and now file, geodatabases. However, our desire to endorse open file formats is overshadowed by our need to prescribe a database file format that preserves topology, allows long attribute names, and works well within ArcGIS; thus, we specify that spatial data be released in 
Esri's file geodatabase (.gdb) format. To make geologic map data more widely available, we also specify that data be released in shapefile format. We look forward to wider implementation and use of text-based, application-independent delivery formats such as GeoSciML. However, a primary motivation for specifying a standard schema (GeMS) is fully satisfied through a proprietary format; more specifically, organizational efficiency is gained by managing a large number of GIS files in the same format. When that format is anticipated to become obsolete, bulk migration to a new format can be efficiently achieved.

Text not contained in the database should be stored as plain text (.txt), Web Markup Language (.htm/.html), Open Document Format (.odt, ISO/IEC 26300:2006 or its successor), .docx, or publication-formatted Portable Document Format (.pdf) files, and these files should be managed with the GIS data. Tables may be stored in a wide variety of text formats (.csv, .dat, .txt, dbf, .ods, or .xls) or as XML (.xml) files, which most modern database software can import. Note that the venerable dBASE III (.dbf) format, integral to Esri shapefiles, has been abandoned by most other software developers and so is unchanging and, thus, is a reliable choice; apparently, no published standard for the .dbf format exists, but documentation of the format is readily available. For raster images, the patent on the lossless LZW compression format (commonly used for .tif or .gif files) has expired, and patents that may have restricted use of the lossy JPEG compression format (resulting in .jpg images) have been found to be invalid; thus, the choice between .png (another lossless compression format), .tif, .jpg, and .gif files for raster images should depend on technical considerations. Vector, or mixed vector-raster, images can be stored as .pdf or .svg files.

\section{Required, As-Needed, and Optional Elements of a Digital Geologic Map Publication}

For a digital geologic map publication named "mapXYZ", the publication package typically includes the elements listed in table 3. The digital data package is essential and shall include the files identified in tables 4 and 5. As-needed elements are mandatory only if they are part of the content of the map report; for example, if a figure 1 is on the map sheet or in the pamphlet, then a figure1.png file (or its equivalent) must be present in the digital data package. Optional elements may or may not be present, at the discretion of the author or publisher. These elements are further discussed in the sections that follow (and in appendix 2).

\section{The Database Design}

As with the overall digital data package, some geologic map database elements are required, some are as-needed, and some are optional (fig. 1). Required and as-needed elements are specified below. Optional elements are described in appendix 2; note that additional optional elements are permitted by this schema.
For each element (that is, each feature dataset, feature class, or nonspatial table), we provide a name, identify the element type, and enumerate the fields (attributes) in the relevant table. Unless otherwise noted, all fields are of data type text ( = string). For each field, we briefly discuss content and domains where appropriate. For some elements, this is followed by a short example table and some further discussion.

\section{General Considerations}

\section{This Design Uses a Relational Database}

This database design relies on relations (joins, or relationship classes) between the various feature classes and nonspatial tables. Examples of these relations include the following (the type of relation is shown in brackets):

- From the MapUnit field in MapUnitPolys feature class to the MapUnit field in DescriptionOfMapUnits table [many-to-one].

- From all $x x x$ SourceID fields (for example, DataSourceID, LocationSourceID, AnalysisSourceID, DefinitionSourceID) in all feature classes and some nonspatial tables to the DataSources_ID field in the DataSources table [many-to-one].

- From the Type, ExistenceConfidence, IdentityConfidence, ScientificConfidence fields, etc., in all feature classes and some nonspatial tables to the Term field in the Glossary table [many-to-one].

Figures 2,3, and 4 show relations among the elements of this database design.

\section{Field Hygiene}

- GeMS does not, in general, prescribe the lengths of text fields; field lengths need to be long enough to encompass all values but short enough to not unduly inflate the size of the database.

- It is important that required fields not be deleted, even if all values in the field are null (that is, they have no content). Deletion of required fields would create unnecessary ambiguity: questions such as "Is this a GeMS-compliant database?" or "Is the database incomplete or corrupted?" would certainly arise.

- When a particular instance (row value) of a field is intended to have no content, it should be set to $<$ null $>$ if the underlying database software supports an explicit $<$ null $>$ value. If the software does not support a $<$ null $>$ value, then enter a text string: use "\#null" for text fields that are long (that is, $\geq 5$ characters); use "\#” for short ( $<5$ characters) text fields. For numeric fields, do not use 0 ; use either -9 or -9999 (but be consistent; do not use both -9 and -9999 for null values in the same numeric field). 
Table 3. Elements of a digital geologic map publication named "mapXYZ".

[Abbreviation: FGDC, Federal Geographic Data Committee]

\begin{tabular}{|c|c|}
\hline Filename & Comments \\
\hline \multicolumn{2}{|r|}{ Required elements } \\
\hline mapXYZ.pdf & Map graphic (high resolution; publication quality) \\
\hline mapXYZ-browse.png (or .jpg, .tif) & Browse graphic or thumbnail of map (should be a small file) \\
\hline mapXYZ-metadata.xml & $\begin{array}{l}\text { FGDC-compliant metadata for the overall map publication; additional inclusion of metadata in a more } \\
\text { readable form (for example, txt, } . \mathrm{html} \text { ) as a supplementary file is recommended }\end{array}$ \\
\hline mapXYZ-gdb.zip & $\begin{array}{l}\text { Zip archive containing the map database and other elements of the digital data package (see table } 4 \\
\text { below for contents) }\end{array}$ \\
\hline mapXYZ-open.zip & Open shapefile version of database (see "Shapefile Version of the Database" section below for contents) \\
\hline \multicolumn{2}{|r|}{ As-needed elements } \\
\hline mapXYZ-pamphlet.pdf & Map pamphlet (fully formatted; publication quality) \\
\hline
\end{tabular}

Table 4. Required, as-needed, and optional contents of the zip archive (that is, the "mapXYZ-gdb.zip" file) that contains the database and other elements of the digital data package for a geologic map publication named "mapXYZ".

[Abbreviation: FGDC, Federal Geographic Data Committee]

\begin{tabular}{ll}
\hline \multicolumn{1}{c}{ Filename } & \multicolumn{1}{c}{ Required contents } \\
\hline mapXYZ.gdb (folder) & Folder that constitutes the map database \\
mapXYZ.mxd & ArcMap document stored using relative pathnames and including relevant macros \\
mapXYZ-metadata.xml & FGDC-compliant metadata for the overall map publication (copy of file referenced in table 3 above) \\
resources (folder) & $\begin{array}{l}\text { Folder of digital resources that accompanies map database in a digital geologic map publication } \\
\text { (see table 5 below for contents) }\end{array}$ \\
\hline & \multicolumn{1}{c}{ As-needed contents } \\
\hline mapXYZ-pamphlet.pdf & Map pamphlet (copy of file referenced in table 3 above) \\
mapXYZ-base.gdb (folder) & Folder with base-map data (required if not published elsewhere) \\
\hline & \multicolumn{1}{c}{ Optional contents } \\
\hline mapXYZ.pmf & ArcReader document \\
\hline
\end{tabular}

Table 5. Required, as-needed, and optional contents of the resources folder that accompanies the database in a digital geologic map publication named "mapXYZ".

[Abbreviation: FGDC, Federal Geographic Data Committee]

\begin{tabular}{|c|c|}
\hline Filename & Comments \\
\hline \multicolumn{2}{|r|}{ Required contents } \\
\hline mapXYZ.style & $\begin{array}{l}\text { ArcGIS .style file that contains the area, line, and point symbols used to symbolize the map. Must } \\
\text { include all symbols specified in database. It is recommended that the .style contain a subset of the } \\
\text { symbols in the FGDC cartographic standard; please see Resources on the GeMS website (https:// } \\
\text { ngmdb.usgs.gov/Info/standards/GeMS/) for a suggested master .style file. Note that a .style file is not } \\
\text { necessary if Esri cartographic representations are encoded in the database itself }\end{array}$ \\
\hline \multicolumn{2}{|r|}{ As-needed contents } \\
\hline CMU (as .pdf or .png file) & $\begin{array}{l}\text { Graphic representation of the Correlation of Map Units (CMU) diagram. Needed only if (1) CMU } \\
\text { is present in report and (2) CMU is not encoded within the map database }\end{array}$ \\
\hline $\begin{array}{l}\text { figures (.png, .pdf, .jpeg, .gif, or .tif } \\
\text { files) }\end{array}$ & Must be numbered as in report; may be included as individual files or gathered into a folder \\
\hline
\end{tabular}


- Fields should not be repurposed to hold other types of information. To store other attribute information in a table, you must add other fields. As noted below, such extensions to the GeMS database schema are encouraged.

- The values in certain fields must be defined in the Glossary table. These fields are highlighted in magenta bold type in the tables that follow.

- For most feature classes, a Notes field is prescribed; however, populating this field is always optional, and it may be omitted if desired.

- All feature classes and nonspatial tables contain a primary key field that has a name in the form of TableName ID. When values of this primary key populate a field in another feature class or nonspatial table, that field must have a different name. For example, values of DataSources_ID populate fields named DescriptionSourceID (in the DescriptionOfMapUnits table), LocationSourceID (in point data tables), and DataSourceID (in many tables). Tables 6, 7, 8, and 9 show examples of these relations in various data tables.

Note that, for the foliation measurement example in table 9 (OrientationPoints_ID = "ORP02"), the location was determined by one worker (LocationSourceID = "DAS4"), and the orientation of foliation was measured by another (OrientationSourceID $=$ "DAS3"). The map author chose to list these as two separate sources in the DataSources table (see table 6). Alternatively, the author could have chosen to combine both OrientationSourceID and LocationSourceID into a third source, for example, "DAS5" (Source = "fieldwork by A.B. Geolog and C.D. Nagt, 2014"; see table 6). See further discussion in the "DataSources (Nonspatial Table)" section below.

If data loaded into the database do not already have user-managed primary keys, we suggest that primary key values be created from a three-letter prefix that is based on an abbreviation of the name of the containing table (for example, in the MapUnitPolys feature class, use the abbreviation "MUP" as the prefix for values of MapUnitPolys_ID [see table 12]; in the ContactsAndFaults feature class, use "CAF" for values of ContactsAndFaults_ID [see table 13]), combined with an integer suffix that is unique in the containing table. The suffix could be the text representation of the Esri database-maintained ObjectID that is included in all feature classes and nonspatial tables (for example, a row that has ObjectID $=27$ in the MapUnitPolys feature class could have MapUnitPolys_ID = "MUP027"). Following this pattern provides unique identifiers for all features within the database as well as easily understood foreign keys.

\section{Type, MapUnit, Label, and Symbol Fields}

Most feature classes in the GeMS schema contain the fields Type, Label, and Symbol; however, the MapUnitPolys polygon feature class contains the field MapUnit in lieu of the Type field, as discussed below (see tables 10 and 11 for examples):

\begin{tabular}{|c|c|}
\hline \multicolumn{2}{|l|}{$\boxminus \square$ example.gdb } \\
\hline 曰 它 CorrelationOfMapUnits & optional \\
\hline 드 CMULines & optional \\
\hline 國 CMUMapUnitPolys & optional \\
\hline$\because$ CMUPoints & optional \\
\hline 曰它 CrossSectionA & optional \\
\hline$\dashv$ CSAContactsAndFaults & optional \\
\hline 國 CSAMapUnitPolys & optional \\
\hline$\because$ CSAOrientationPoints & optional \\
\hline$\boxminus$ 它 GeologicMap & required \\
\hline$\rightarrow$ CartographicLines & as-needed \\
\hline 드 ContactsAndFaults & required \\
\hline DataSourcePolys & as-needed \\
\hline$\because$ FossilPoints & as-needed \\
\hline$\because$ GenericPoints & as-needed \\
\hline$\because$ GeochronPoints & as-needed \\
\hline$\succsim$ GeologicLines & as-needed \\
\hline$\neg$ MapUnitLines & as-needed \\
\hline 圖 MapUnitOverlayPolys & as-needed \\
\hline$\because$ MapUnitPoints & as-needed \\
\hline 國 MapUnitPolys & required \\
\hline$\because$ OrientationPoints & as-needed \\
\hline 國 OverlayPolys & as-needed \\
\hline$\because$ Stations & as-needed \\
\hline 国 DataSources & required \\
\hline 国 DescriptionOfMapUnits & required \\
\hline 国 GeoMaterialDict & required \\
\hline 国 Glossary & required \\
\hline MiscellaneousMaplnformation & optional \\
\hline 国 RepurposedSymbols & as-needed \\
\hline 覧 StandardLithology & optional \\
\hline
\end{tabular}

Figure 1. ArcCatalog view of the contents of a GeMS-structured database, showing required, as-needed, and optional database elements. As-needed elements must be present in the database if they are part of the content of a map report. Optional elements may or may not be present, at the discretion of the author and publisher. Note that the feature dataset for a single cross section is named CrossSectionA; additional cross sections are named CrossSectionB, CrossSectionC, and so on.

- The Type field specifies what kind of feature is represented (see table 10). For example, a line within the ContactsAndFaults feature class could be a "contact", "thrust fault", or "water boundary"; a point within a GeochronPoints feature class could represent the location of a potassium-argon date.

- The MapUnit field in the MapUnitPolys feature class (see table 11) is the equivalent of the Type field in other feature classes, in that it specifies what map unit is represented (for example, "Qal", "Tg", "Ks", "Trdu"). The value of MapUnit is commonly an ASCII-character ${ }^{12}$ equivalent of the map-unit label; see discussion of the Label field below.

\footnotetext{
${ }^{12} \mathrm{ASCII}$, American Standard Code for Information Interchange; ASCII characters mainly consist of lower and upper case letters, numerals, and certain punctuation characters (specifically, ones that can be accessed using the Shift key).
} 


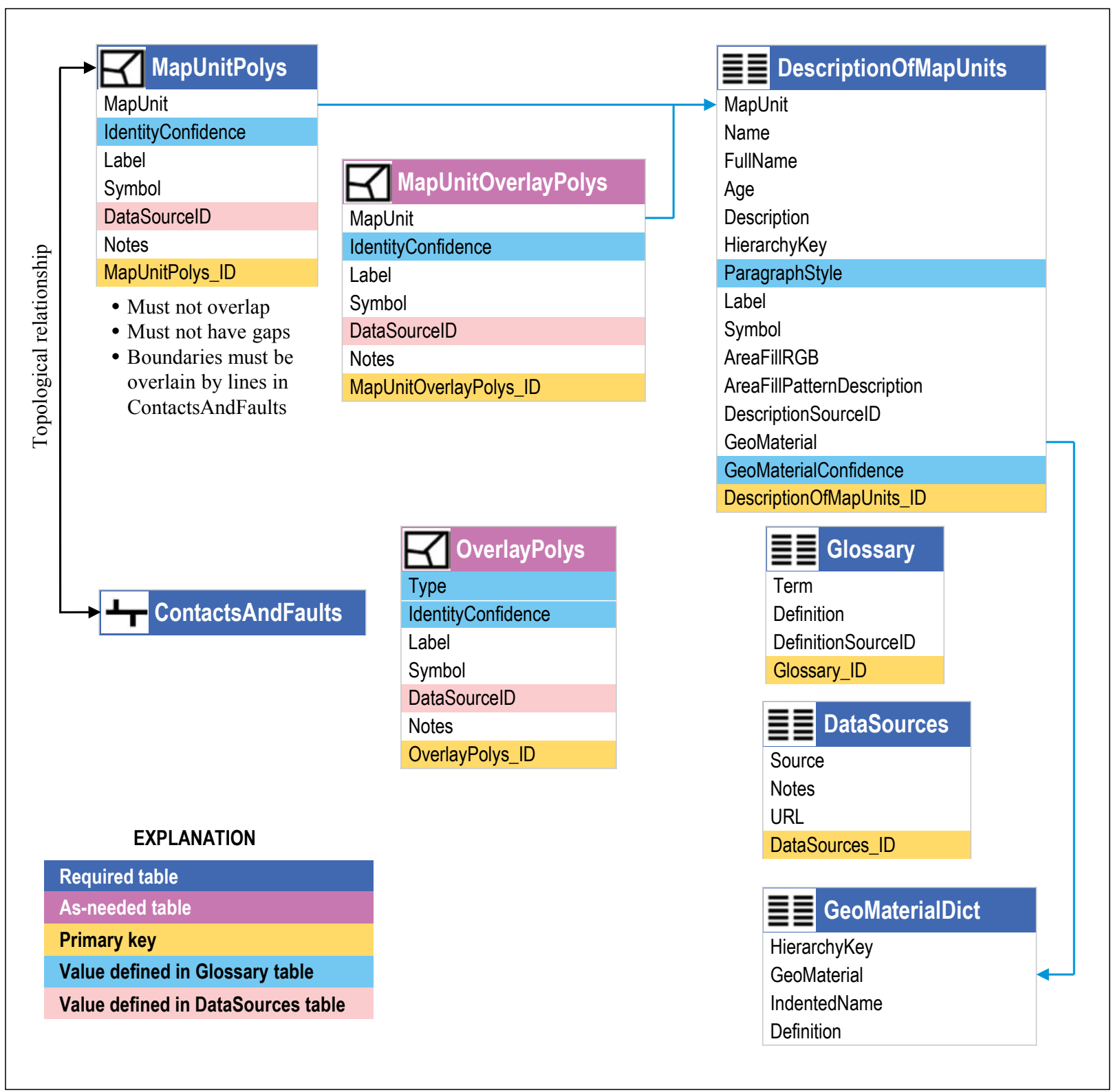

Figure 2. Entity-relationship diagram for selected polygon feature classes and associated nonspatial tables, highlighting required and as-needed database elements. Also highlighted are primary keys, values defined in Glossary table, and values defined in DataSources table. Black arrow shows topological relationship between MapUnitPolys and ContactsAndFaults feature classes; blue arrows show other foreign key relationships. Topology rules (bulleted items) are listed for polygons in MapUnitPolys feature class.

- The Label field contains the desired annotation for a feature: the annotation may be simple text (for example, "SAN ANDREAS FAULT", "14 Ma"), orin the MapUnitPolys feature class - it is an ASCIIcharacter equivalent of the map-unit label; it may have the same value as MapUnit (for example, "Qal") and have an appended "?" if IdentityConfidence is "questionable", but it will differ if a special font is used for geologic age symbols (for example, “^du”, which results in the map-unit symbol kdu when displayed with the FGDCGeoAge font; see table 11).

- The Symbol field specifies either a point symbol (sometimes called a marker symbol), a line symbol, or an area-fill symbol (a color and (or) pattern fill) for the feature (for example, a dot for a potassium-argon age locality; a heavy black line for a fault; a pale-yellow color fill for a polygon of alluvium) (see tables 10 and 11). 


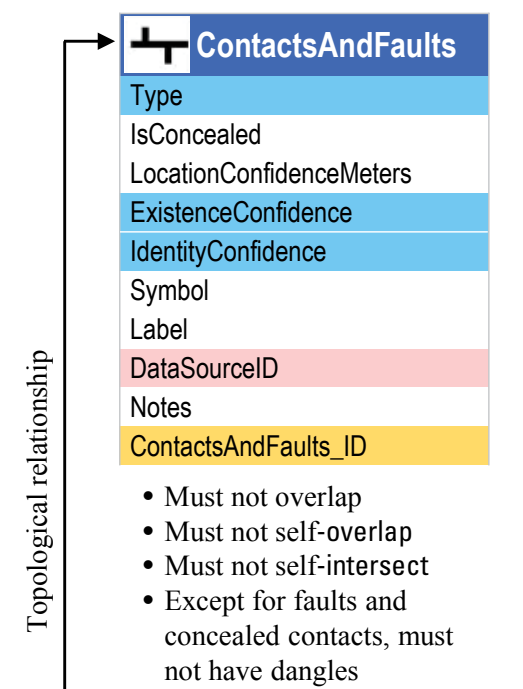

EXPLANATION

Required table

As-needed table

Primary key

Value defined in Glossary table

Value defined in DataSources table

\begin{tabular}{|l|}
\hline $\mathbf{L}$ CartographicLines \\
\hline Type \\
Symbol \\
Label \\
DataSourcelD \\
Notes \\
CartographicLines_ID \\
\hline
\end{tabular}

\begin{tabular}{|l|}
\hline $\mathbf{L}$ GeologicLines \\
\hline Type \\
\hline IsConcealed \\
LocationConfidenceMeters \\
\hline ExistenceConfidence \\
\hline IdentityConfidence \\
\hline Symbol \\
Label \\
\hline DataSourcelD \\
\hline Notes \\
GeologicLInes_ID \\
\hline
\end{tabular}

- Must not self-overlap

- Must not self-intersect

\begin{tabular}{|l|}
\hline \multicolumn{1}{|c|}{ IsoValueLines } \\
\hline Type \\
\hline Value \\
ValueConfidence \\
Symbol \\
Label \\
\hline DataSourcelD \\
Notes \\
IsoValueLines_ID \\
\hline
\end{tabular}

E三 DataSources

\section{Source}

Notes

URL

DataSources_ID

㭅 Glossary

Term

Definition

DefinitionSourcelD

Glossary_ID

\section{无无 DescriptionOfMapUnits}

\begin{tabular}{|l|l|}
\hline MapUnit \\
Name \\
FullName \\
Age \\
Description \\
HierarchyKey \\
\hline ParagraphStyle \\
\hline Label \\
Symbol \\
AreaFillRGB \\
AreaFillPatternDescription \\
DescriptionSourcelD \\
GeoMaterial \\
\hline GeoMaterialConfidence \\
DescriptionOfMapUnits_ID \\
\hline
\end{tabular}

\begin{tabular}{|l|}
\hline MapUnit MapUnitLines \\
\hline IsConcealed \\
LocationConfidenceMeters \\
ExistenceConfidence \\
IdentityConfidence \\
\hline Symbol \\
Label \\
PlotAtScale \\
DataSourcelD \\
Notes \\
MapUnitLines_ID \\
- Must not overlap \\
- Must not self-overlap \\
- Must not intersect \\
- Must not self-intersect
\end{tabular}

Figure 3. Entity-relationship diagram for selected line feature classes and associated nonspatial tables, highlighting required and as-needed database elements. Also highlighted are primary keys, values defined in Glossary table, and values defined in DataSources table. Black arrow shows topological relationship between ContactsAndFaults and MapUnitPolys feature classes; blue arrow shows other foreign key relationship. Topology rules (bulleted items) are listed for lines in ContactsAndFaults, GeologicLines, and MapUnitLines feature classes. 


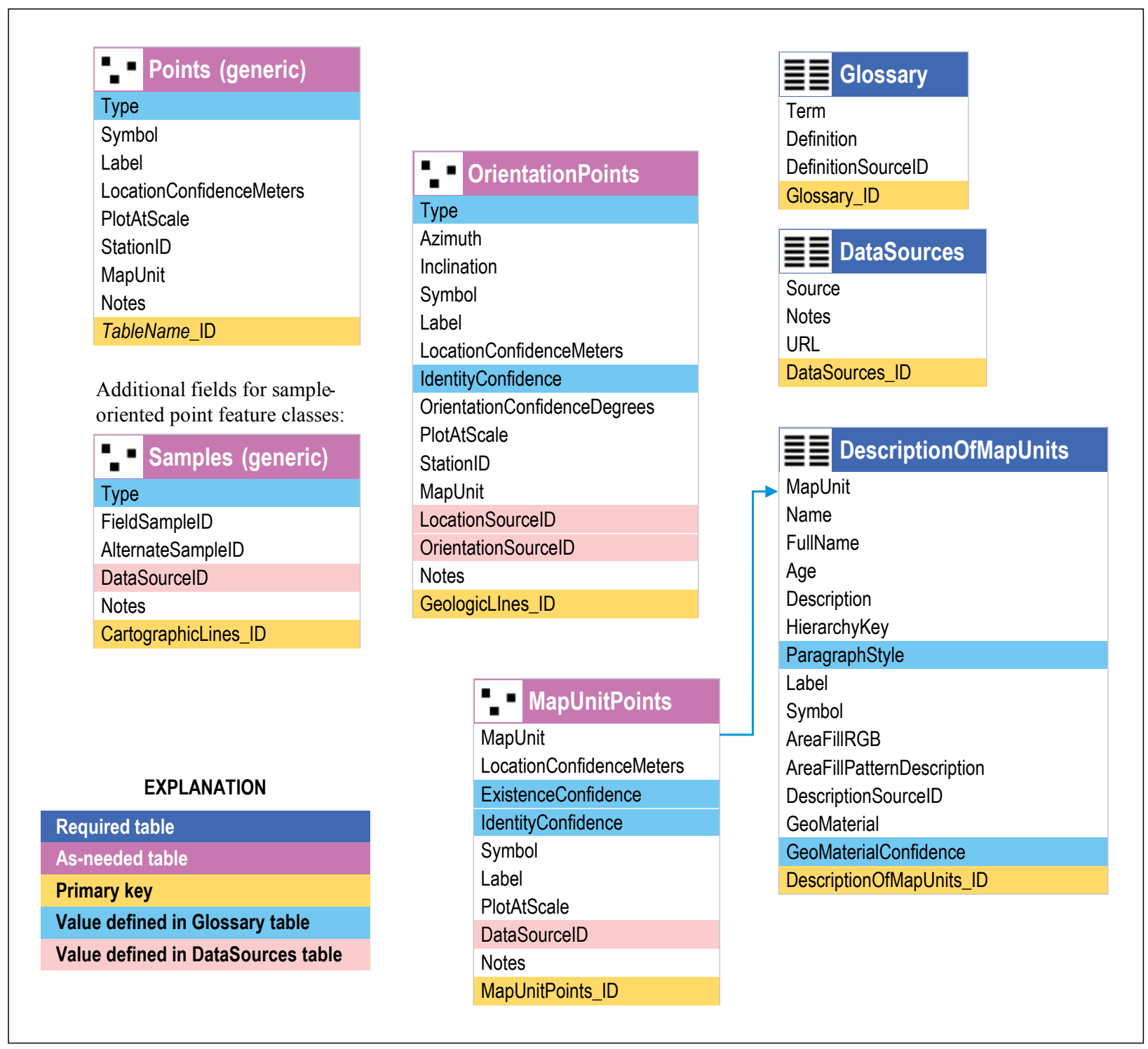

Figure 4. Entity-relationship diagram for selected point feature classes and associated nonspatial tables, highlighting required and as-needed database elements. Also highlighted are primary keys, values defined in Glossary table, and values defined in DataSources table. Blue arrow shows foreign key relationship.

This three-fold division of what, at first glance, may seem to be one entity is necessary because (1) it is desirable for the database to provide a preferred visualization of the map data, and (2) there generally is not a one-to-one correspondence between values of Type (or MapUnit), Symbol, and Label:

- For MapUnitPolys, values of Label will differ from those of MapUnit when special symbols are used to specify geologic ages (for example, the Label value of "^du", which results in the map-unit symbol kdu when displayed with the FGDCGeoAge font, is different from the MapUnit value of "Trdu"; see table 11).
- For MapUnitPolys, values of Label may also reflect values of the IdentityConfidence field (that is, queries may be added).

- For lines in ContactsAndFaults, values of Symbol typically reflect the combination of values of Type, IsConcealed, and the confidence fields.

- Faults that have the same Type value likely will have different names (for example, "San Andreas Fault", "Hayward Fault") and, thus, different values of Label. 
Table 6. Examples of selected fields (and their values) in a DataSources table.

[See also, tables 15,16$]$

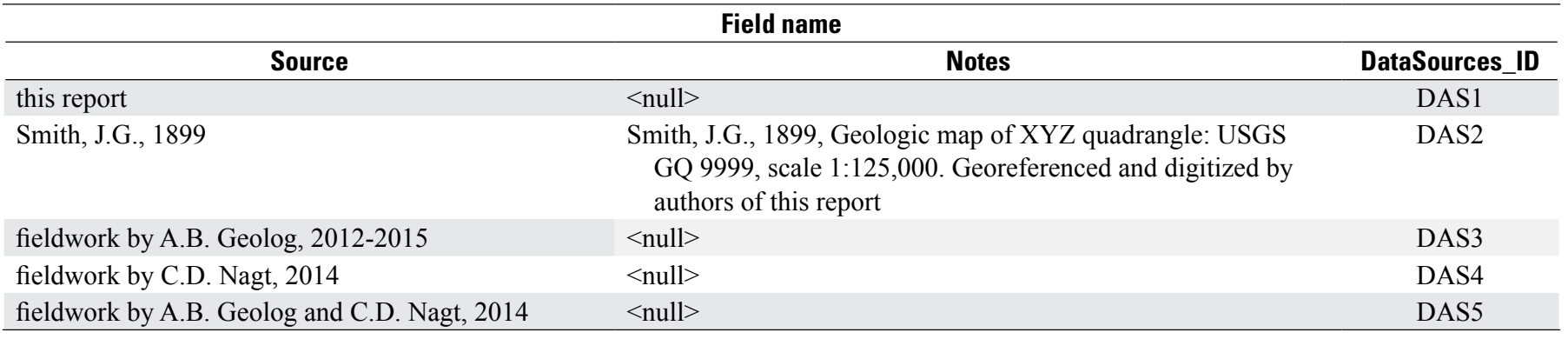

Table 7. Examples of selected fields (and their values) in a DescriptionOfMapUnits table.

[See also, table 14]

\begin{tabular}{lllrr}
\hline \multicolumn{3}{c}{ Field name } \\
\hline \multicolumn{1}{c}{ MapUnit } & \multicolumn{1}{c}{ Name } & DescriptionSourcelD & DescriptionOfMapUnits_ID \\
\hline Qal & Alluvium & DAS1 & DMU1 \\
Qgd & Glacial drift & DAS1 & DMU2 \\
Trg & Triassic granitic rocks & DAS1 & DMU3 \\
\hline
\end{tabular}

Table 8. Examples of selected fields (and their values) in a ContactsAndFaults feature class.

[Abbreviations: N, no; Y, yes. See also, table 13]

\begin{tabular}{|c|c|c|c|}
\hline \multicolumn{4}{|c|}{ Field name } \\
\hline Type & IsConcealed & DataSourceID & ContactsAndFaults_ID \\
\hline contact & $\mathrm{N}$ & DAS1 & CAF001 \\
\hline
\end{tabular}

Table 9. Examples of selected fields (and their values) in an OrientationPoints feature class.

[See also, table 22]

\begin{tabular}{lccccc}
\hline & \multicolumn{5}{c}{ Field name } \\
\hline & Type & Azimuth & LocationSourcelD & OrientationSourcelD & OrientationPoints_ID \\
\hline bedding & 73 & DAS3 & DAS3 & ORP01 \\
foliation & 120 & DAS4 & DAS3 & ORP02 \\
\hline
\end{tabular}

\section{Polygons, Lines, and Topology—What Goes Where?}

By convention, a geologic map depicts the distribution of earth materials on a particular map horizon, usually the Earth's surface. Map-unit polygons (which can also include areas of open water, permanent snowfields, glaciers, and unmapped areas) are bounded by contacts, faults, shorelines, snowfield or glacier boundaries, scratch boundaries (that is, boundaries for which no line is drawn), and (or) the map boundary. Contacts do not normally separate polygons of the same map unit, although faults commonly do. In addition, a map-unit polygon may be partly bisected by a fault whose trace terminates within that polygon (that is, a dangling fault in GIS jargon).

The distribution of units on a particular map is recorded in the polygon feature class MapUnitPolys. Contacts that separate map units, faults that bound map units, and dangling faults are recorded in the line feature class ContactsAndFaults. Elements of these feature classes are involved in topological relations that are described below. Elements are assigned to these feature classes to simplify enforcement of topological relations when constructing a database, as well as to facilitate topological queries when using the database. 
Table 10. Examples of values in the Type, Symbol, and Label fields in a point feature class and the resulting map images.

[See also, table 11]

\begin{tabular}{|c|c|c|c|c|}
\hline \multicolumn{3}{|c|}{ Database field name ${ }^{1}$} & \multicolumn{2}{|c|}{ Map image } \\
\hline Type & Symbol $^{2}$ & Label & Symbol & Label $^{3}$ \\
\hline radiocarbon & 31.21 & $14,020 \pm 45^{[4]}$ & • & $14,020 \pm 45$ \\
\hline inclined upright bedding & 06.13 & $30^{[5]}$ & 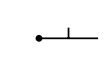 & 30 \\
\hline vertical bedding & 06.03 & $<$ null $>^{[6]}$ & + & \\
\hline
\end{tabular}

${ }^{1}$ All values in the database fields are of data type $=$ text.

${ }^{2}$ Values are indices to symbols chosen from the Geological Survey of Canada's implementation of the FGDC standard (Federal Geographic Data Committee, 2006) as an Esri style (Everett and Dohar, 2012). This .style file is available under the Resources heading at the GeMS website (https://ngmdb.usgs.gov/Info/standards/GeMS/).

${ }^{3}$ For improved legibility, sizes of map-image labels shown here are larger than would appear on a map.

${ }^{4}$ In this example, the value in the Label field (radiocarbon age, in years before present) is calculated from values in the NumericalAge, AgePlusError, and AgeMinusError fields in the GeochronPoints feature class.

${ }^{5}$ In this example, the value in the Label field (dip of inclined bedding) is derived from the Inclination field in the OrientationPoints feature class.

${ }^{6}$ In this example, the value in the Label field is $<$ null $>$ because no dip value is labeled on the map (vertical bedding, by definition, has a vertical dip of $90^{\circ}$; by convention, a vertical $\left[90^{\circ}\right]$ dip is not labeled on the map).

Table 11. Examples of values in the MapUnit, IdentityConfidence, Symbol, and Label fields in the MapUnitPolys feature class and the resulting map images.

$[$ See also, tables 10,12$]$

\begin{tabular}{|c|c|c|c|c|c|}
\hline \multicolumn{4}{|c|}{ Database field name $^{1}$} & \multicolumn{2}{|c|}{ Map image } \\
\hline MapUnit & IdentityConfidence & Symbol $^{2}$ & Label $^{3}$ & Symbol & Label $^{4}$ \\
\hline Qal & certain & 60 & Qal & & Qal \\
\hline $\operatorname{Trdu}^{[5,6]}$ & certain & 730 & $\wedge d u^{[7]}$ & & kdu \\
\hline Qls & questionable & 90 & Qls? ${ }^{[8]}$ & & Qls? \\
\hline
\end{tabular}

${ }^{1}$ All values in the database fields are of data type $=$ text.

${ }^{2}$ Values are indices to colors selected from the FGDC cartographic standard's CMYK Color Chart (Federal Geographic Data Committee, 2006). An ArcGIS .style file ("FGDCcmyk.style") of this chart is available under the Resources heading at the GeMS website (https://ngmdb.usgs.gov/Info/standards/GeMS/).

${ }^{3}$ Values are determined from the IdentityConfidence value in the MapUnitPolys feature class combined with the corresponding value of Label in the DescriptionOfMapUnits table.

${ }^{4}$ Map-image labels are displayed using the FGDCGeoAge font (U.S. Geological Survey, 2006; available at https://pubs.usgs.gov/tm/2006/11 A02/). An OpenType version ("FGDCGeoAge.otf") of this font is available under the Resources heading at the GeMS website (https://ngmdb.usgs.gov/Info/standards/ GeMS/). For improved legibility, sizes of map-image labels shown here are larger than normally would appear on a map.

5" $\mathrm{Tr}$ " is an easily understood ASCII-character identifier for the geologic age symbol $\mathrm{k}$ (= Triassic).

${ }^{6} \mathrm{ASCII}$, American Standard Code for Information Interchange; ASCII characters mainly consist of lower and upper case letters, numerals, and certain punctuation characters (specifically, ones that can be accessed using the Shift key).

${ }^{7}$ Character "^” (Shift-6) in the FGDCGeoAge font results in the geologic age symbol k (= Triassic).

${ }^{8}$ If IdentityConfidence = "questionable", then "?" should be appended to the Label value. 
Some maps show contacts and faults that are concealed beneath covering map units (for example, beneath thin surficial deposits or open water). These concealed contacts and faults are recorded in the line feature class ContactsAndFaults, and their concealed status should be encoded as IsConcealed $=$ "Y". Such concealed contacts and faults may or may not be involved in topology with MapUnit polygons; in addition, concealed contacts and faults may dangle.

Many geologic maps contain other feature classes that are not involved fully in map topology (for example, fossil localities, fold axes, or bedding-orientation measurements). Feature classes for such features are described in the "As-Needed Elements" section below.

Some database producers may choose to create polygons and edit linework in the absence of a topology relationship class. For instance, rather than using the topology editing tools to synchronously edit boundaries that are shared between line and polygon feature classes, users may prefer to create the line features (boundaries) first and then construct the polygons as (or after) their bounding lines are finished. For the purposes of this data-delivery design, the method used to produce the feature classes does not matter; it only matters that the feature classes in the published database follow the topology rules outlined for each feature class.

\section{Directional Lines}

Many types of geologic lines have directionality, equivalent to handedness. Examples are thrust faults, which (by convention) have sawteeth on the side of the upper (overlying) plate, and normal faults, which (also by convention) have half-circle or ball-and-bar decorations on the downthrown (overlying) side of the fault. The direction that the decorations point indicates the geometry of that feature.

To preserve this directionality in the database, we subscribe to the right-hand rule for such line features: that is, lines should be created or edited such that the decoration points to the right while one travels from the start of the line segment to its end. In the case of up-down (U-D) notation on faults, the direction that has the right side of the fault in the down (D) direction should be considered as the right-hand direction.

\section{Required Elements}

\section{GeologicMap (Feature Dataset)}

The GeologicMap feature dataset is nearly equivalent to the map graphic in that it contains all the geologic content within the neatline, with the exception of the base map. All elements in a feature dataset share a single geospatial reference framework.

Listed below are the required elements in a GeologicMap feature dataset, along with tables that describe the content of each element and (if applicable) a list of topology rules.

\section{MapUnitPolys (Polygon Feature Class)}

The MapUnitPolys polygon feature class contains all the units within the mapped area: geologic map units, areas of open water, permanent snowfields, or glaciers, as well as any unmapped areas. Table 12 describes the fields (and their values) in MapUnitPolys.

Some units use specialized symbols in their map-unit labels; however, the special characters needed to render such symbols on a map are less intelligible to readers of the MapUnit field (see examples in table 11). Thus, the MapUnit value is not used to label the unit on the map; instead, the Label field is. The Label field accommodates the use of special characters (for example, to display geologic age symbols using the FGDCGeoAge font; see table 11). The MapUnit field generally contains an easily understood ASCII-character equivalent of the specialized symbol. When no specialized symbol is needed, Label and MapUnit typically have the same value (see table 11). For more information on geologic age symbols, see Section 32 in the FGDC cartographic standard (FGDC, 2006).

Note that areas of open water (for example, lakes, wide rivers), glaciers, snowfields, and unmapped areas must be included in MapUnitPolys, and they must have non-null MapUnit values (examples would be MapUnit = "water", "glacier", "unmapped"); however, such areas commonly are not labeled with their MapUnit values (therefore, Label $=<$ null $>$ ).

Topology Rules

The following topology rules apply to the MapUnitPolys polygon feature class:

- Polygons must not overlap.

- No gaps can be present between polygons.

- Polygon boundaries must be overlain by (topologically, not simply cartographically) lines in the ContactsAndFaults feature class.

\section{ContactsAndFaults (Line Feature Class)}

The ContactsAndFaults line feature class contains the types of lines that bound map-unit polygons; common types are contacts, faults, waterlines, and map boundaries. Table 13 describes the fields (and their values) in ContactsAndFaults.

Map boundaries, open-water boundaries, and snowfield and glacier boundaries delineate the edge of certain map-unit polygons. In this sense, they are contacts, and, therefore, they are included in this feature class.

Scratch boundaries (also known as wash boundaries) are boundaries for which no symbol is drawn. We recommend setting the value of Symbol = "blank" for scratch boundaries.

Examples of values suggested for the Type field include the following:

"contact"

"contact, internal"

"contact, gradational"

"contact, unconformable" 
Table 12. Fields in MapUnitPolys (a polygon feature class in the GeologicMap feature dataset).

[Content of fields in magenta type (in this case, the IdentityConfidence field) must be defined in Glossary table. See also, tables 11, 14]

\begin{tabular}{|c|c|c|}
\hline Field name & Description & Notes \\
\hline MapUnit & $\begin{array}{l}\text { Short, easily understood ASCII-character }{ }^{1} \\
\text { identifier for the map unit represented } \\
\text { by this polygon. Foreign key to } \\
\text { DescriptionOfMapUnits table (see } \\
\text { table 14) }\end{array}$ & $\begin{array}{l}\text { Examples of values are "Qal", "Tg", "Kit", "Trdu”, "water" (see table 11). Use } \\
\text { of special characters is discouraged. Null values not permitted }\end{array}$ \\
\hline IdentityConfidence & $\begin{array}{l}\text { Indicates how confidently this polygon } \\
\text { has been identified as a particular } \\
\text { map unit }\end{array}$ & $\begin{array}{l}\text { Typical values are "certain", "questionable", "unspecified". Values must } \\
\text { be defined in Glossary table. Null values not permitted; suggest setting } \\
\text { default value = "certain" }\end{array}$ \\
\hline Label & $\begin{array}{l}\text { Describes text label for this map-unit } \\
\text { polygon }\end{array}$ & $\begin{array}{l}\text { May or may not be same as MapUnit value. Determined from value of } \\
\text { Label in DescriptionOfMapUnits table and IdentityConfidence (that is, } \\
\text { if IdentityConfidence = "questionable", then "?" is appended to Label } \\
\text { value in DescriptionOfMapUnits table). Allows for special fonts to show } \\
\text { geologic age symbols (see table 11) and other non-ASCII characters. } \\
\text { Null values permitted }\end{array}$ \\
\hline DataSourceID & $\begin{array}{l}\text { Identifies source of each data element. } \\
\text { Foreign key to DataSources table }\end{array}$ & Null values not permitted \\
\hline Notes & Optional field & Free text for additional information specific to this polygon. Null values permitted \\
\hline MapUnitPolys_ID & Primary key & $\begin{array}{l}\text { Examples of values are "MUP1", "MUP2". Values must be unique in } \\
\text { database. Null values not permitted }\end{array}$ \\
\hline
\end{tabular}

${ }^{1}$ ASCII, American Standard Code for Information Interchange; ASCII characters mainly consist of lower and upper case letters, numerals, and certain punctuation characters (specifically, ones that can be accessed using the Shift key).

"fault"

"fault, normal"

"fault, reverse"

"fault, thrust"

"scratch boundary"

"glacier boundary"

"waterline"

"shoreline"

"map boundary", "map neatline", or "neatline"

The above list is derived from Sections 1, 2, 30, and 31 in the appendix of the FGDC cartographic standard (FGDC, 2006). Other values of Type certainly are acceptable (for example, see the CGI FaultType and ContactType vocabularies, available at http://resource.geosciml.org/def/voc/).

It has been a common practice to encode (via symbolization on a paper map, or a single attribute field in a database) contacts as, for example, "contact, certain", "contact, inferred", "contact, approximately located", "contact, concealed", "contact, queried", or some permutation of these values. In the GeMS schema, these line features all would have the same value of Type ("contact") but different values of IsConcealed, LocationConfidenceMeters, ExistenceConfidence, and (or) IdentityConfidence. Faults and some other line features that have been encoded in a similar fashion are treated the same way in the GeMS schema. Although this is a departure from what may have been a common practice, it allows for map data that is more easily communicated to nonspecialists, is more intelligently combined with other data, and is better used at other scales. See discussion in the "Feature-Level Metadata" section above.

Note that not all lines in ContactsAndFaults necessarily bound polygons. In some cases, polygons separated by concealed faults or concealed contacts may have been merged during construction of the database; also, some faults and concealed contacts may dangle (that is, terminate within a polygon) and, thus, may not always separate polygons.

\section{Topology Rules}

The following topology rules apply to the ContactsAndFaults line feature class:

- Lines must not overlap each other.

- Lines must not overlap themselves.

- Lines must not intersect themselves.

- Except for faults and concealed contacts, lines must not have dangles; therefore, dangling lines must have values of either (1) Type = "fault" (or some variant thereof) or (2) Type $=$ "contact" or "fault" and IsConcealed = "Y". 
Table 13. Fields in ContactsAndFaults (a line feature class in the GeologicMap feature dataset).

[Abbreviations: N, no; Y, yes. Content of fields in magenta type (in this case, the Type, ExistenceConfidence, and IdentityConfidence fields) must be defined in Glossary table. See also, table 8]

\begin{tabular}{|c|c|c|}
\hline Field name & Description & Notes \\
\hline Type & $\begin{array}{l}\text { Specifies type of geologic feature } \\
\text { represented by this database row }\end{array}$ & $\begin{array}{l}\text { Examples of values are "contact", "fault", "thrust fault", "waterline”, } \\
\text { "glacier boundary", "map boundary". Values must be defined in } \\
\text { Glossary table. Null values not permitted }\end{array}$ \\
\hline LocationConfidenceMeters & $\begin{array}{l}\text { Half-width (in meters) of } \\
\text { positional-uncertainty envelope } \\
\text { around this line feature }\end{array}$ & $\begin{array}{l}\text { Data type }=\text { float. Null values not permitted; recommend setting } \\
\text { value }=-9 \text { if value is not known (see discussion above in } \\
\text { "Feature-Level Metadata" and example values in table 1) }\end{array}$ \\
\hline ExistenceConfidence & $\begin{array}{l}\text { Indicates how confidently existence of } \\
\text { this line feature has been determined }\end{array}$ & $\begin{array}{l}\text { Typical values are "certain", "questionable", "unspecified". Values } \\
\text { must be defined in Glossary table. Null values not permitted; } \\
\text { suggest setting default value = "certain" }\end{array}$ \\
\hline IdentityConfidence & $\begin{array}{l}\text { Indicates how confidently this line } \\
\text { feature has been identified as a } \\
\text { particular type }\end{array}$ & $\begin{array}{l}\text { Typical values are "certain", "questionable", "unspecified". Values } \\
\text { must be defined in Glossary table. Null values not permitted; } \\
\text { suggest setting default value = "certain" }\end{array}$ \\
\hline Symbol & References a line symbol & $\begin{array}{l}\text { Line symbols must be included in accompanying .style file. } \\
\text { Null values (which would indicate lines are not shown on } \\
\text { map) permitted; if Esri cartographic representations are used } \\
\text { to symbolize lines, value may be null. Determined from } \\
\text { values of Type, IsConcealed, LocationConfidenceMeters, } \\
\text { ExistenceConfidence, and IdentityConfidence fields, as well as } \\
\text { map display scale }\end{array}$ \\
\hline DataSourceID & $\begin{array}{l}\text { Identifies source of each data element. } \\
\text { Foreign key to DataSources table }\end{array}$ & Null values not permitted \\
\hline Notes & Optional field & $\begin{array}{l}\text { Free text for additional information specific to this line feature. } \\
\text { Null values permitted }\end{array}$ \\
\hline
\end{tabular}

\section{DescriptionOfMapUnits (Nonspatial Table)}

DescriptionOfMapUnits is a nonspatial table that captures the content of the Description of Map Units (DMU) included in a geologic map report. Table 14 describes the fields (and their values) in the DescriptionOfMapUnits table.

Note that the authoritative source for the content of a DescriptionOfMapUnits table should always be a DMU (not a List of Map Units, or LMU), whether the DMU appears on the map sheet or is included in an accompanying pamphlet. An LMU on a map sheet is only a distillation of the full content of the DMU and is provided as a convenience to help identify units on the map.

A DMU is strongly formatted and typically hierarchical, in many cases following the guidelines and conventions specified in USGS's "Suggestions to Authors of the Reports of the United States Geological Survey, Eighth Edition" (STA8) (USGS, in press; see also, STA7, Hansen, 1991, p. 49-52); see discussion in the "Notes on the Content of the Description
Field" section below. The DescriptionOfMapUnits table encodes the DMU as is specified in STA8, without loss of information and - with two exceptions (the two GeoMaterial fields) - without imposing additional structure or content.

We have added the fields GeoMaterial and GeoMaterialConfidence to the DescriptionOfMapUnits table in order to provide abbreviated content that will allow for simple queries and (or) generalized symbolizations of the character of geologic map units across one or many maps. It is important that users populate the GeoMaterial field with terms from the NGMDB standard term list (included in appendix 1); they should then use the GeoMaterialConfidence field to characterize the relative appropriateness of the term chosen for the GeoMaterial field. Note that the abbreviated content in the GeoMaterial field is not a replacement for the full, robust, free-text information in the Description field. Note also that additional, more detailed lithologic information may be included either in a supplementary user-defined table or in the optional StandardLithology table (see discussion in appendix 2, "Optional Elements"). 


\section{Notes on the Content of the Description Field}

The content of the Description field in the DescriptionOfMapUnits table is an essential part of this database schema, just as the map-unit descriptions in a DMU are always an essential part of a geologic map report. This schema does not prescribe a particular form or content of such descriptions; however, we support the ideas put forth in STA8 (USGS, in press), which states the following:

"Description of Map Units", ***[13] for each map unit, contains its map-unit label, its name, and its assigned age system, followed by an abbreviated account of the lithologic characteristics of its rocks and (or) deposits *** described in order of increasing age; thus, surficial units are described before bedrock units. The ranking of units and subunits on a geologic map is indicated in the DMU by successive levels of indentation of the unit descriptions ${ }^{* * *}$, as the following examples show:

Dakota Group (Cretaceous) - Alternating sandstone and dark-colored shale beds overlying beds of sandstone, conglomerate, and claystone

\section{South Platte Formation (Lower Cretaceous) -}

Alternating beds of gray to black shale and brown-weathering sandstone

Kassler Sandstone Member-Brownweathering, massive, fine-grained sandstone

Headings are used to organize and identify groups of units. Beneath each heading, each map unit is described in order of increasing age; thus, the upper member of a formation would be described before its middle or lower members. The hierarchy of a DMU heading is indicated by a unique type style; *** the DMU title ("Description of Map Units") is [considered a] first-order heading. A typical DMU contains at least two second-order headings (for example, "Surficial Deposits" and "Bedrock"), although most DMUs commonly contain a few third- and fourth-order headings as well (for example, "Volcanic Rocks" and "Pyroclastic Deposits"); some maps may require as many as seven orders of headings.

STA8 (USGS, in press) also notes the long tradition of writing the DMU and makes suggestions for its content, some of which may be useful to transcribe here:

[A] unit description always includes the following three fundamental elements: (1) the map-unit label (for example, Tml), (2) the name of the unit (for example, Monterey Formation), and (3) the unit's age or stratigraphic position, listed from youngest to oldest (for example, Pliocene and Miocene). These elements

\footnotetext{
${ }^{13}$ An ellipsis $(* * *)$ indicates where text has been omitted from the quotation.
}

are followed by the unit description, which contains information such as lithologic content, color, grain size, bedding characteristics, porosity, permeability, fracture characteristics, mineral or fossil content, remanent magnetization, and thickness. Correlation with other units, the nature of a unit's upper and lower contacts, any radiometric or other types of age determinations, the sources of specific information, and, if applicable, more detailed stratigraphic ages $* * *$ also may be provided. Stratigraphic or lithologic details are limited to data from within the map area, although a brief discussion of correlations with rock units in nearby areas may be appropriate. Reference citations to other published works also may be included.

Map-unit descriptions in a DMU typically are written in an abbreviated, telegraphic style in which complete sentences are not necessary. Nonessential articles ("a," "an," "the") usually are omitted. A notable exception is that the article "the" is retained before a map-unit name. In many DMU sentences, the word "unit" (in singular form) is the implicit subject and commonly is omitted; however, retaining the word "unit" is recommended for clarity (rather than using a unit symbol) when referring to a unit within its own description. Sentences may also begin with a specific subject, such as "Deposits," "Beds," or "Flows," if desired.

The order of describing lithology may differ from map to map, but it should be consistent within a given DMU. Features such as color, permeability, and gradations in grain size usually modify the lithologic term. Commas are used to separate all strings of descriptive terms except the final one before the lithologic term, as the following example shows:

Curtis Formation (Jurassic) - Consists of interbedded light-gray, fine- to coarse-grained, poorly sorted, thickly bedded sandstone, pale-green shale, and pale-blue limestone

To separate ideas within a description, periods or semicolons may work better than conjunctions. Lengthy unit descriptions may be divided into paragraphs. Periods are omitted at the end of each description, except when it is split into paragraphs (in such cases, a period is included at the end of every paragraph except the last one). If entries are fairly short and no one lithology predominates, normal word order (for example, "pale-green shale and pale-blue limestone") may read more smoothly than inverted order, but if an entry is long and has a string of modifiers, an inverted sentence structure may be easier to follow:

Curtis Formation (Jurassic) - Interbedded sandstone, shale, and limestone. Sandstone, light-gray, fine- to coarse-grained, poorly sorted, 
Table 14. Fields in DescriptionOfMapUnits (a nonspatial table).

[Abbreviations: DMU, Description of Map Units; HTML, Hypertext Markup Language; NGMDB, National Geologic Map Database. Content of fields in magenta type (in this case, the ParagraphStyle and GeoMaterialConfidence fields) must be defined in Glossary table. See also, tables 7, 11, 12, 3-1]

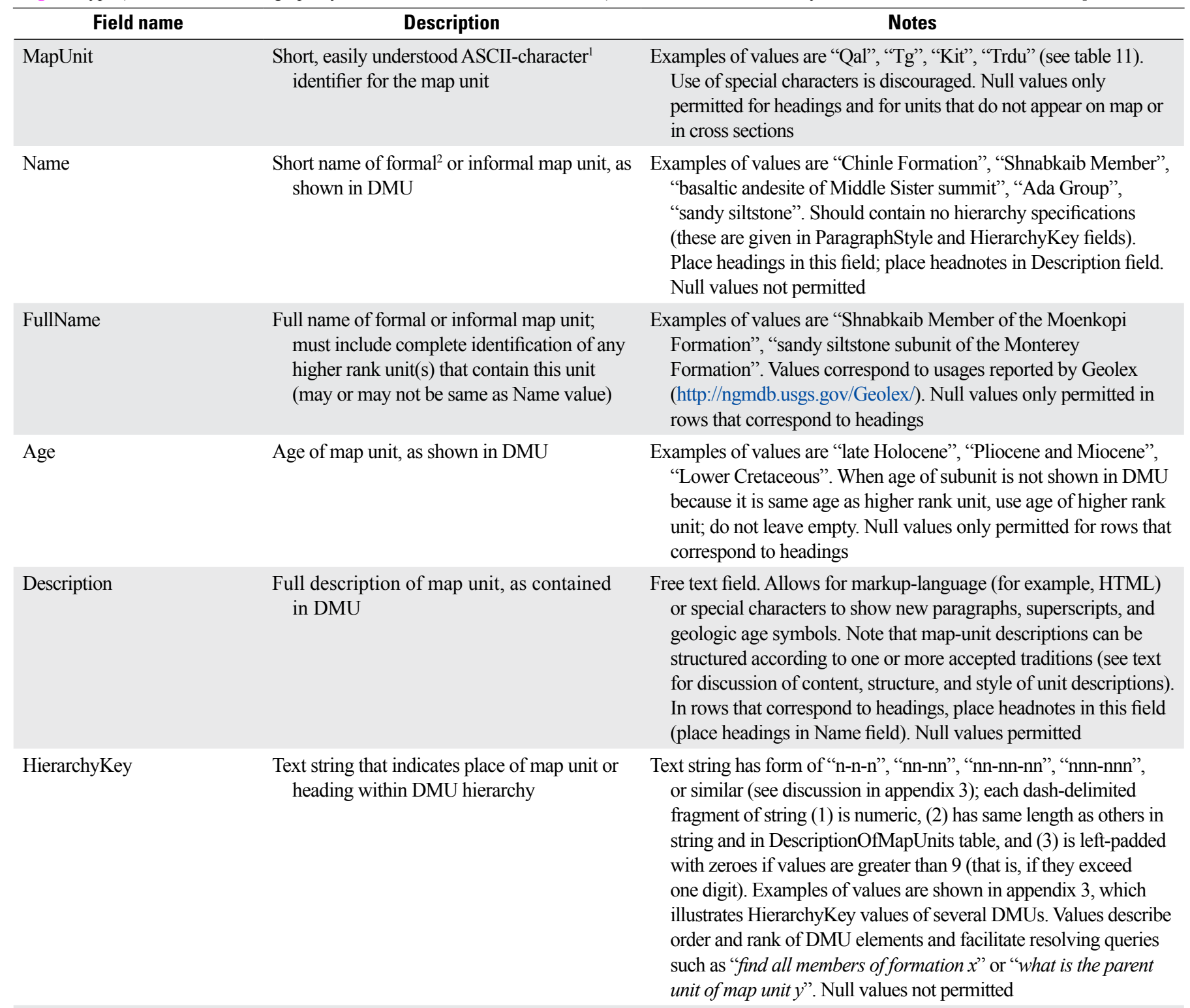

ParagraphStyle $\begin{aligned} & \text { Name of user-defined paragraph style that } \\ & \text { indicates hierarchy of map units and } \\ & \text { headings within DMU }\end{aligned}$
indicates hierarchy of map units and headings within DMU
Examples of values are "DMUHeading1", "DMUHeading2", "DMUUnit1", "DMUUnit2". Values must be defined in Glossary table; definition typically includes formatting specifications (for example, in Glossary table for Term = "DMUUnit1", value for Definition might be Definition = "Paragraph style for formatted description of first-rank map unit. Times New Roman, Regular, 10 pt, 12 pt leading, left justified, $66 \mathrm{pt}$ left indent, $-20 \mathrm{pt} 1 \mathrm{st}$ line indent, $3 \mathrm{pt}$ space before"). Null values not permitted

Label

Describes text label for map-unit polygons. Field from which map-unit label is generated
May or may not be same as MapUnit value. Allows for special fonts to show geologic age symbols and other non-ASCII characters (see table 11). Null values only permitted for headings, for units that are not labeled on map or cross sections, and for units that do not appear on map or cross sections (for example, water, glaciers, overlay units, and units that are represented on map only by their subunits) 
Table 14.-Continued

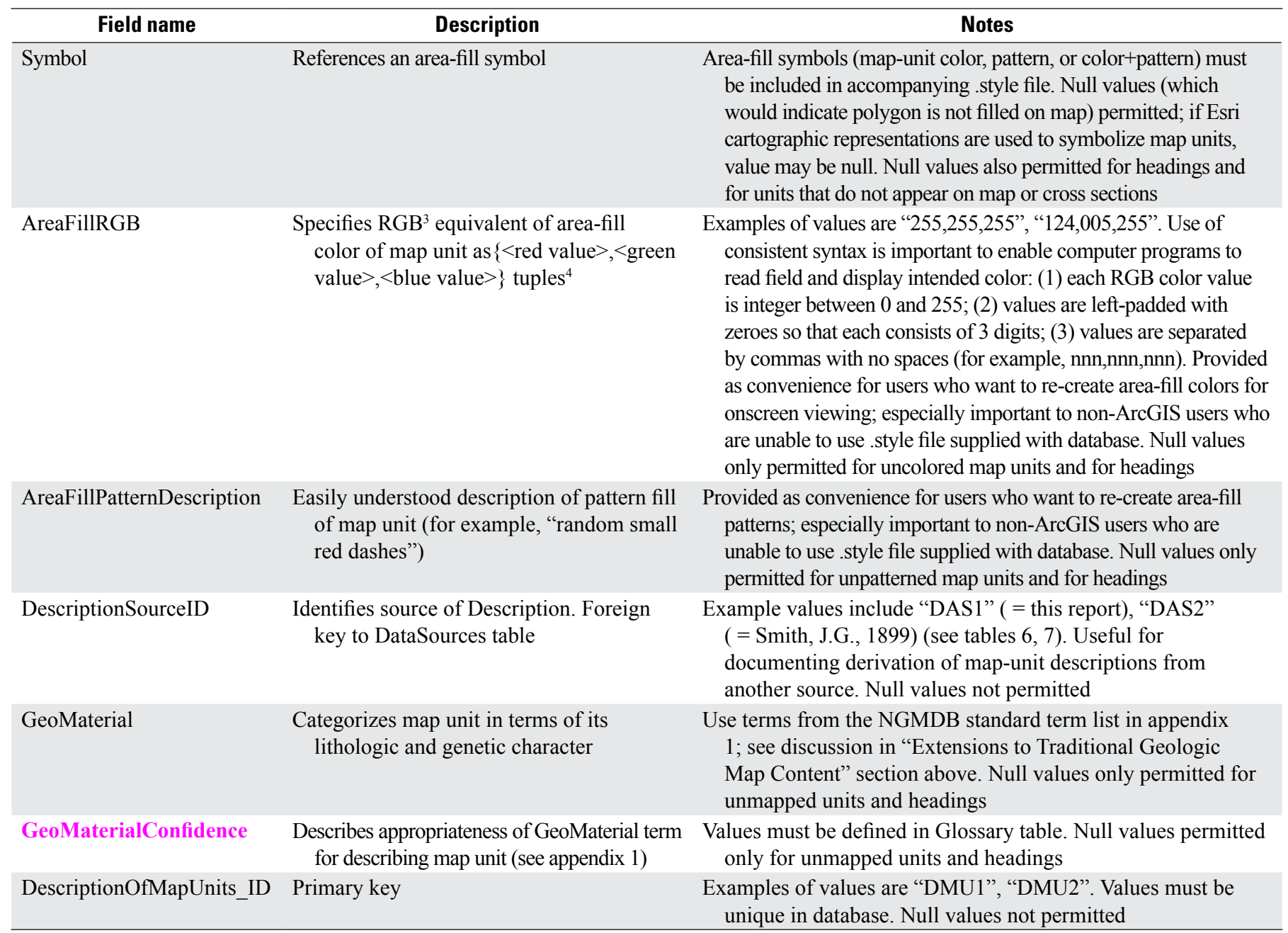

${ }^{1}$ ASCII, American Standard Code for Information Interchange; ASCII characters mainly consist of lower and upper case letters, numerals, and certain punctuation characters (specifically, ones that can be accessed using the Shift key).

${ }^{2}$ Note that formal names should be verified in the U.S. Geologic Names Lexicon (Geolex; see https://ngmdb.usgs.gov/Geolex/); if your usage does not agree with that in Geolex, please contact the Geologic Names Committee secretary (at gems@usgs.gov).

${ }^{3} \mathrm{RGB}=$ red/green/blue color space, used in computer monitors and televisions.

${ }^{4} \mathrm{~A}$ tuple is a sequence (or ordered list) of elements.

and thickly bedded. Shale, pale-green to gray and fine- to medium-bedded. Limestone, pale-blue to light-gray, fine-grained, and medium-bedded

Other information that characterizes the entire unit (for example, magnetization, fossil or mineral content, radiometric age, and so on) usually follows. The order in which such features are listed may depend on their significance in the mind of the author, but the style should be consistent throughout the description.

Although not specifically prescribed in STA8 (USGS, in press), we submit that listing DMU elements in a consistent manner facilitates map comprehension. To that end, we offer R.E. Wells' (USGS, written commun., 2010) suggestion to use the following basic order for listing elements in map-unit descriptions in a DMU: lithology (dominant and subordinate); color; induration; grain size; mineralogy; bedding characteristics; interpreted facies; unit thickness; nature of contacts; fossil content/stage/zone; magnetic polarity; correlation with other units; radiometric age; and data sources where needed.

We note that not all map units in a DMU need all of these descriptive elements; also, volcanic rocks, plutonic rocks, metamorphic rocks, and surficial deposits require somewhat different approaches. Nevertheless, the main elements as articulated by Wells are relevant for most map units. We further note that map-unit descriptions in a DMU should emphasize the essential character of each unit and how it is distinguished in the field from nearby units. In poorly exposed terrain, it can be a challenge to write a map-unit description that accurately expresses no more than what little can be seen or is known about a map unit. 


\section{Populating the DescriptionOfMapUnits Table}

All map units (including units in cross sections) in the DMU need an entry in the DescriptionOfMapUnits table. Any map units shown as overlays need entries as well. Entries should also include map units that traditionally are not listed in the DMU, such as "water", "glacier", or "unmapped area", as well as all geologic units that are listed as parent units in the DMU but are not represented as polygons on the map or in the cross sections.

Headings and headnotes in the DMU also need entries in the DescriptionOfMapUnits table: headings are stored in the Name field, and headnotes are stored in the Description field. An example of the translation of part of a DMU into a DescriptionOfMapUnits table is provided in appendix 3.

The DescriptionSourceID field commonly points to Source values $=$ "this report" or "modified from < earlier report $>$ ".

\section{Order and Rank of Map Units}

The order and rank of map units and headings in a DMU carry a significant amount of information. In the printed DMU, order is explicit and rank is expressed by font and paragraph style. In the DescriptionOfMapUnits table, where order is easily lost by sorting and text is unformatted, order and rank are expressed by values in the HierarchyKey field. Examples of the content of the HierarchyKey field and its construction are provided in appendix 3.

We wish to enable generation of the formatted text DMU from the DescriptionOfMapUnits table, both to facilitate report production and to test that the database is capturing essential content in the report. In most cases, font and paragraph style in the printed DMU can be calculated from values in HierarchyKey and MapUnit (the latter to test whether a DescriptionOfMapUnits row corresponds to a mapped unit or is a heading). However, we have found that (1) such automated text generation requires links to the appropriate parts of a document template, and (2) additional information is needed to distinguish use of a group or formation as a heading from its use as an unmapped parent unit (that is, only its subunits have been mapped). The ParagraphStyle field is included to meet these needs (see table 14 for an example of values). In most — but not all — cases, ParagraphStyle values can be calculated from values of HierarchyKey and MapUnit. The consequent partial redundancy between HierarchyKey and ParagraphStyle fields allows for some automated checking of the DescriptionOfMapUnits table for logical consistency.

As a practical matter, values in the ParagraphStyle field and their corresponding definitions in the Glossary table should correspond to the formatting styles prescribed by an organization's publication standards and guidelines. If templates for these styles are available, they may be incorporated into a script that automates much — if not all —of the construction of a formatted DMU from the DescriptionOfMapUnits table.

\section{DataSources (Nonspatial Table)}

DataSources is a nonspatial table that identifies the sources for the content of the geologic map database. Table 15 describes the fields (and their values) in the DataSources table, and table 16 provides some example records.

All features and table entries must be associated with a data source. For maps that contain all new information and use a single vocabulary source, the DataSources table will be very short. For compilations that contain data from many sources that have been modified and (or) reinterpreted so that the data source has effectively been changed, the DataSources table will become longer and quite useful. It is worth noting that GeMS does not prescribe the level of detail or depth of provenance that is contained in the DataSources table. We recognize that the amount or level of source lineage recorded on a feature-level basis often is a function of the time and resources available, and so it is a decision that needs to be made by the mapper or compiler and the publishing agency. Some may choose to only cite either publication references or "this report"; others may cite only the original report in which the data first appeared; still others may record the person compiling the map and the year as the data source because they may have a tool that easily captures this data. All are acceptable in the GeMS schema, although their actual usefulness may vary.

\section{GeoMaterialDict (Nonspatial Table)}

GeoMaterialDict is a nonspatial table that provides definitions and a hierarchy for GeoMaterial names prescribed by the GeMS database schema. Table 17 describes the fields (and their values) in the GeoMaterialDict table.

The GeoMaterialDict table will be installed automatically in any database created with the script GeMS_CreateDatabase Arc10.py (available at https://github.com/usgs/GeMS_Tools) or its replacement. Note that users of the GeMS schema do not create this table; furthermore, this table should not be modified by authors or publishers of individual maps.

\section{Glossary (Nonspatial Table)}

Glossary is a nonspatial table that provides definitions for some of the terminology used in a GeMS database. Table 18 describes the fields (and their values) in the Glossary table, and table 19 provides some examples of its records. Note that values in the Term field must have unique definitions within the database because they are used as foreign keys to the Glossary table. For example, use of "fold axis" as a Type term in GeologicLines, with a definition of "intersection of fold axial plane with the Earth's surface", and "fold axis" as a Type term in OrientationPoints, with a definition of "orientation of fold hinge", is not possible (instead, one might consider using "minor fold axis" or something similar as the Type term for the point feature). Terminology that requires definition in the Glossary table includes all values in the Type, ExistenceConfidence, IdentityConfidence, ScientificConfidence, ParagraphStyle, and AgeUnits fields. Terminology used only in the Description field of the DescriptionOfMapUnits table should not be defined here.

If no intellectual property restrictions exist, it is permissible (and recommended) to replicate all or parts of an external 
Table 15. Fields in DataSources (a nonspatial table).

[See also, tables 6, 16]

\begin{tabular}{lll}
\hline \multicolumn{1}{c}{ Field name } & \multicolumn{1}{c}{ Description } & Notes \\
\hline Source & $\begin{array}{c}\text { Short, terse, easily understood } \\
\text { ASCII-character identifier } \\
\text { for a data source }\end{array}$ & $\begin{array}{c}\text { Contains brief citation. For DataSources_ID = "DAS1", Source = "this report" (by } \\
\text { convention). If source is another publication, provide full reference citation in Notes field. } \\
\text { Null values not permitted }\end{array}$ \\
Notes & Optional field & $\begin{array}{c}\text { Free text for additional information specific to this data source. Provide full reference } \\
\text { citation(s) here (if applicable). Processing or data acquisition procedures may be } \\
\text { described here. Null values permitted }\end{array}$ \\
URL & Primary key & $\begin{array}{c}\text { Contains link, either online URL or Digital Object Identifier (DOI), to either data source or } \\
\text { full description of data source. Null values permitted } \\
\text { Examples of values are "DAS1", "DAS2". Values must be unique in database. Null values } \\
\text { not permitted }\end{array}$ \\
\hline
\end{tabular}

${ }^{1}$ ASCII, American Standard Code for Information Interchange; ASCII characters mainly consist of lower and upper case letters, numerals, and certain punctuation characters (specifically, ones that can be accessed using the Shift key).

Table 16. Examples of records in a DataSources table.

[Abbreviations: DEM, digital elevation model; ft, foot (feet); K-Ar, potassium-argon; PP, Professional Paper; USGS, U.S. Geological Survey. See also, tables 6, 15]

\begin{tabular}{|c|c|c|}
\hline Source & Notes & DataSources_ID \\
\hline this report & $\begin{array}{l}\text { field compilation automated by A. Digitdroid, using georeferenced scan of } \\
\text { greenline mylar, Esri ArcScan tools, and manual editing }\end{array}$ & DAS1 \\
\hline this report, interpreted from 6-ft lidar DEM & data acquired winter 2003-2004 by Puget Sound Lidar Consortium & DAS2 \\
\hline this report, Ralph Haugerud field data, 2005 & & DAS3 \\
\hline USGS PP 901 & $\begin{array}{l}\text { King, P.B., and Beikman, H.M., 1974, Explanatory text to accompany the geologic map } \\
\text { of the United States: U.S. Geological Survey Professional Paper 901, } 40 \text { p. }\end{array}$ & DAS4 \\
\hline Beta Laboratories, Report 1999-451 & $\begin{array}{l}\text { K-Ar dates determined using constants from Steiger, R.H., and Jager, E., 1977, } \\
\text { Subcommission on geochronology: Convention on the use of decay constants in } \\
\text { geo and cosmochronology: Earth and Planetary Science Letters, v. 36, p. 359-362 }\end{array}$ & DAS6 \\
\hline Jackson, 1997 & $\begin{array}{l}\text { cited in Glossary table for sources of term definitions. Jackson, J.A., ed., 1997, Glossary } \\
\text { of Geology (4th ed.): Alexandria, Va., American Geological Institute, } 657 \text { p. }\end{array}$ & DAS7 \\
\hline
\end{tabular}

Table 17. Fields in GeoMaterialDict (a nonspatial table).

[Abbreviation: DMU, Description of Map Units. See also, table 14]

\begin{tabular}{|c|c|c|}
\hline Field name & Description & Notes \\
\hline HierarchyKey & $\begin{array}{l}\text { Text string that indicates } \\
\text { hierarchy of entries within } \\
\text { list of GeoMaterial terms }\end{array}$ & $\begin{array}{l}\text { Text string has form of "n-n-n", "nn-nn", "nn-nn-nn", "nnn-nnn", or similar (see discussion } \\
\text { in appendix 3); each dash-delimited fragment of string (1) is numeric, (2) has same length } \\
\text { as others in string and in DescriptionOfMapUnits table, and (3) is left-padded with zeroes } \\
\text { if values are greater than } 9 \text { (that is, if they exceed one digit). Examples of values are shown } \\
\text { in appendix 3, which illustrates HierarchyKey values of several DMUs. Documents, for } \\
\text { example, that "limestone" (HierarchyKey = "01-02-02-01") is a kind of "carbonate rock" } \\
\text { (HierarchyKey = "01-02-02"), which is a kind of "sedimentary rock" (HierarchyKey = } \\
\text { "01-02"), which is a kind of "sedimentary material" (HierarchyKey = "01") }\end{array}$ \\
\hline GeoMaterial & Name of GeoMaterial unit & Terms used to populate GeoMaterial field in DescriptionOfMapUnits table \\
\hline IndentedName & $\begin{array}{l}\text { GeoMaterial name } \\
\text { with indentation that } \\
\text { corresponds to rank of } \\
\text { entry within hierarchy }\end{array}$ & Useful for visually portraying hierarchy of GeoMaterial terms \\
\hline
\end{tabular}


Figure 18. Fields in Glossary (a nonspatial table).

[Abbreviation: K-Ar, potassium-argon. See also, table 19]

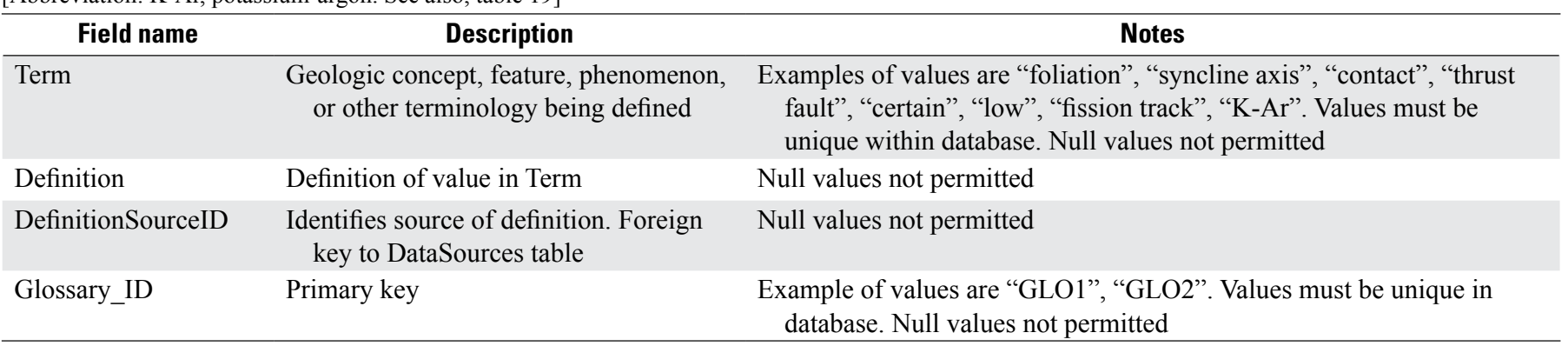

Table 19. Examples of records in a Glossary table.

[See also, table 18]

\begin{tabular}{|c|c|c|c|}
\hline Term & Definition & DefinitionSourcelD & Glossary_ID \\
\hline contact & $\begin{array}{l}\text { Line denoting unfaulted [depositional, intrusive, metamorphic, etc.] boundary } \\
\text { between two geologic map units }\end{array}$ & DAS1 & GLO01 \\
\hline
\end{tabular}

glossary in the Glossary table, but please provide appropriate credit for the definitions in the DataSources table by means of the DefinitionSourceID field. If such restrictions preclude replicating a definition in the Glossary table, the term should still be listed in the table, along with (if possible) a brief, paraphrased definition and a note that refers the user to the publication cited in the definitionsource record.

\section{As-Needed Elements}

Some geologic maps contain features other than contacts, faults, and map-unit polygons. If such features are present in a geologic map report, they should be digitally encoded in the map database. If such elements are not present, the corresponding feature classes need not be included in the database; therefore, these feature classes are as-needed elements. Examples of these types of features are foliation, lineation, and bedding measurements; various sample localities for fossil, geochemical, and geochronological analyses; localities of field photographs; fold axes (or, more precisely, traces of fold-hinge surfaces); structure contours; concentration contours; former ice limits and ice-flow lines; cross-section lines; and areas of mineralization or manmade fill (both commonly depicted as overlays).

Many such types of features exist, and there are many ways to partition these types of features into feature classes. At one extreme, each feature type can be represented by a separate feature class, in which case, the Type field of that feature class is redundant. At the other extreme, all feature types that have the same kind of geometry (that is, point, line, or polygon) can be assigned to a single feature class and then differentiated by the Type attribute. In the latter case, one might be tempted to add a plethora of attribute fields to the feature class; however, many attributes are likely to be left unpopulated for many features. For example, if age-sample localities and bedding measurements were recorded in a single point feature class, sample number would be irrelevant for bedding measurements, and strike and dip would be irrelevant for age-sample localities.

In discussions with colleagues, we have been unable to agree on a best partitioning because different cases of database use suggest different ways of partitioning. And because we see no best partitioning, we do not prescribe the use of particular as-needed feature classes. Instead, we present guidelines for designing and naming feature classes, we discuss principles that govern the structure of point data, and we present several examples of as-needed feature classes. Note that if a GeMS database uses any of the feature classes described here, such feature classes should conform to these descriptions. For example, do not invent your own GeologicLines feature class that has different attributes than the ones we describe; you are free to create and use an additional feature class, but it needs to have a different name.

\section{Guidelines for Naming and Designing Additional Feature Classes}

When naming and designing additional feature classes, follow these guidelines:

- The feature class name emphasizes the identity of the feature class.

- The feature class name includes one of the terms "Points", "Lines", or "Polys" (as appropriate), except when it 
would be redundant (for example, ContactsAndFaults, not ContactsAndFaultsLines, and Stations, not StationPoints).

- Feature class names and field names typically are compound words written in PascalCase.

- Feature class names and field names do not contain spaces or underscores, with one exception (see next guideline).

- Every feature class has a primary key field named FeatureClassName_ID (note that this is the sole exception to the previous no underscores guideline).

- Every feature class has at least one sourceID field. If each feature has a single source, this field typically is named DataSourceID; if the data source is compound (for example, sample analyses commonly have different sources for the sample location and for the associated sample analysis), there will be multiple sourceID fields (for example, LocationSourceID and AnalysisSourceID).

- The fields ExistenceConfidence, IdentityConfidence, LocationConfidenceMeters, and similar confidence fields are included as appropriate.

- All fields that contain measured attributes, or attributes that represent real-world quantities (for example, strike, dip, concentration, location confidence), are data type = float; note that it may be necessary to define conventions for representing null values (for example, value $=-9$ ) and then document these conventions in the feature-class metadata.

- All attributes of a feature class should be populated for most features: if a feature class has one or more attributes that are not applicable to some subset of features in the class, consider placing those features in a separate feature class that has a more appropriate subset of attributes.

- Consider combining small feature classes that have a common attribute structure.

In the sections that follow, we discuss the basic structure of point data and point feature classes in general, and we describe four examples of as-needed point feature classes ("OrientationPoints", "GeochronPoints", "Stations", and "MapUnitPoints"). We also discuss the as-needed feature classes "GeologicLines", "MapUnitLines", "CartographicLines", "IsoValueLines", "MapUnitOverlayPolys", "OverlayPolys", and "DataSourcePolys". Other possible as-needed feature classes might include "GeochemPoints", "PhotoPoints", "FieldNotePoints", "SamplePoints", "FossilPoints", "FoldLines", and "DikeLines".

\section{Structure of Point Data}

Observations of structure orientations and mineral occurrences, as well as collections of samples for geochemical, paleontologic, geochronologic, and other kinds of analyses, are made at field stations. Two modes are possible for representing such observations, localities, and related analyses and their accompanying locations:
- A normalized mode, in which (1) a "Stations" feature class stores location information and data specific to the station, (2) a nonspatial "Sample" table stores information on samples related to stations, and (3) other nonspatial tables store observations and analyses, one for each observation or analysis type, related to either a sample or station.

- A denormalized mode, in which there is a separate feature class for each type of observation or analysis; in some cases, this results in duplicate station-location and sample information.

Each mode has advantages. The first mode allows error-resistant editing of location and sample information (the station data is recorded in only one place) and is well suited for data-management and -archiving systems. The second mode facilitates the symbolization and organization of point data in map layers in a GIS viewing environment that does not require joins or filtering, and it is more convenient for exporting analytical information from a source database by simply copying the relevant feature class.

Because GeMS is designed primarily for publishing, not creating, geologic map data, we endorse the second mode. However, we note that, to create a GeMS-compliant database, it may be useful to start in the first mode by creating a Stations point feature class that has related data tables (including a Samples table) and then, from these, creating the appropriate data-type-specific point feature classes that will be included in the delivery database.

\section{Point Feature Classes in General}

We recommend that typical point feature classes contain certain basic fields (table 20). We also recommend that sample-oriented point feature classes include certain additional fields (table 21). Note that the MapUnit field, whose value is to be obtained by intersection of a point feature class with the MapUnitPolys feature class, is included so that a point feature class may be extracted from its host database and used elsewhere while retaining some geologic context for the point features. Values of MapUnit should not be null, except for points that lie outside the extent of the MapUnitPolys feature class.

\section{Examples of As-Needed Point Feature Classes}

\section{OrientationPoints (Point Feature Class)}

Observations of structure point data (for example, bedding attitudes, foliation attitudes, slip vectors measured at a point) may be recorded in OrientationPoints. Note that where multiple measurements are taken at one locality, each measurement is to be represented by a separate, but coincident, point feature. Table 22 describes the fields (and their values) of an OrientationPoints feature class.

The Type field identifies the kind of feature for which the orientation was measured (for example, bedding, overturned bedding, stretching lineation, open joint, etc.). Definitions 
Table 20. Fields to be included in a typical point feature class.

[Content of fields in magenta type (in this case, the Type field) must be defined in Glossary table. See also, tables 21 through 25]

\begin{tabular}{|c|c|c|}
\hline Field name & Description & Notes \\
\hline Type & $\begin{array}{l}\text { Specifies type of point feature represented } \\
\text { by this data point }\end{array}$ & $\begin{array}{l}\text { Examples of values are "fossil locality", "well". Values must be } \\
\text { defined in Glossary table. Null values not permitted }\end{array}$ \\
\hline Symbol & References a point symbol & $\begin{array}{l}\text { Point symbols must be included in accompanying .style file. } \\
\text { Null values (which would indicate point data are not shown } \\
\text { on map) permitted; if Esri cartographic representations are } \\
\text { used to symbolize point data, value may be null. See table } 10 \\
\text { for examples of Symbol (and Label) fields }\end{array}$ \\
\hline LocationConfidenceMeters & $\begin{array}{l}\text { Radius (in meters) of positional-uncertainty } \\
\text { envelope around this point feature }\end{array}$ & $\begin{array}{l}\text { Data type }=\text { float. Null values not permitted; recommend setting } \\
\text { value }=-9 \text { if value is not known (see discussion above in } \\
\text { "Feature-Level Metadata" and example values in table 1) }\end{array}$ \\
\hline StationsID & Foreign key to Stations point feature class & $\begin{array}{l}\text { Not needed if table represents simple, nonspecific stations (it } \\
\text { would duplicate Stations_ID primary key field). Null values } \\
\text { permitted }\end{array}$ \\
\hline MapUnit & $\begin{array}{l}\text { Records map unit to which this analysis } \\
\text { or observation pertains. Foreign key to } \\
\text { DescriptionOfMapUnits table }\end{array}$ & $\begin{array}{l}\text { Values obtained by intersection with feature class MapUnitPolys. } \\
\text { Null values permitted only for points that lie outside extent of } \\
\text { MapUnitPolys feature class }\end{array}$ \\
\hline Notes & Optional field & $\begin{array}{l}\text { Free text for additional information specific to this point feature. } \\
\text { Null values permitted }\end{array}$ \\
\hline TableName_ID & Primary key & $\begin{array}{l}\text { Examples of values are "TableName1", "TableName2" (substitute } \\
\text { actual table name for "TableName"). Values must be unique in } \\
\text { database. Null values not permitted }\end{array}$ \\
\hline
\end{tabular}

Table 21. Recommended additional fields to be included in a sample-oriented point feature class.

[See also, tables 20, 22, 23, 24]

\begin{tabular}{lll}
\multicolumn{1}{c}{ Field name } & \multicolumn{2}{c}{ Description } \\
\hline FieldSampleID & Sample number given at time of collection & Null values permitted \\
\hline AlternateSampleID & Museum number, lab number, etc. & Null values permitted \\
MaterialAnalyzed & Identifies material that was analyzed & Examples of values are "wood", "whole rock". Null values permitted \\
\hline
\end{tabular}

(included in the Glossary table) of the Type values shall specify the orientation-measurement convention for that Type of feature (for example, strike and dip, trend and plunge, dip direction and dip). Data creators should ensure that multiple measurements taken at a single locality (for example, bedding and cleavage measurements) have the same StationsID value.

\section{GeochronPoints (Point Feature Class)}

Another example of an as-needed point feature class is GeochronPoints, which records locations of samples collected for geochronological analysis, as well as the associated age analyses (table 23).
Analytical data may be represented either by using an ExtendedAttributes table (see NCGMP09 v.1.1 [USGS NCGMP, 2010]) or, if many data are of a single analysis type, by placing them in a user-defined, analysis-specific table such as KArData.

\section{Stations (Point Feature Class)}

If a map author chooses to include station information in a report, we suggest using a Stations feature class that contains the fields listed in table 24 (see also, table 25). Also, having a Stations feature class may be extremely useful during the initial creation of a map database; its contents may later be parsed into separate feature classes if desired. 
Table 22. Fields in OrientationPoints (an as-needed point feature class in the GeologicMap feature dataset).

[Content of fields in magenta type (in this case, the Type and IdentityConfidence fields) must be defined in Glossary table. See also, tables 1, 9, 20, 21]

\begin{tabular}{|c|c|c|}
\hline Field name & Description & Notes \\
\hline Type & $\begin{array}{l}\text { Specifies type of geologic feature } \\
\text { represented by this database row }\end{array}$ & $\begin{array}{l}\text { Examples of values are "bedding", "upright bedding", } \\
\text { "foliation", "horizontal lineation". Values must be defined } \\
\text { in Glossary table. Null values not permitted }\end{array}$ \\
\hline Azimuth & $\begin{array}{l}\text { Strike of planar feature or trend of } \\
\text { linear feature, as measured in degrees } \\
\text { clockwise from geographic North }\end{array}$ & $\begin{array}{l}\text { Data type }=\text { float. Values limited to range from } 0 \text { to } 360 ; \\
\text { horizontal planar features may have any azimuth value. For } \\
\text { planar features, use right-hand rule (that is, dip is to right of } \\
\text { azimuth direction). Null values not permitted }\end{array}$ \\
\hline Label & $\begin{array}{l}\text { Describes text label to accompany this } \\
\text { point symbol }\end{array}$ & $\begin{array}{l}\text { Typically, value is dip or plunge of measured orientation, } \\
\text { rounded to nearest integer and converted to data type = } \\
\text { string. Null values permitted. See table } 10 \text { for examples of } \\
\text { Label (and Symbol) fields }\end{array}$ \\
\hline LocationConfidenceMeters & $\begin{array}{l}\text { Radius (in meters) of positional-uncertainty } \\
\text { envelope around this point feature }\end{array}$ & $\begin{array}{l}\text { Data type }=\text { float. Null values not permitted; recommend } \\
\text { setting value }=-9 \text { if value is not known (see discussion } \\
\text { above in "Feature-Level Metadata"; see also, example } \\
\text { values in table 1) }\end{array}$ \\
\hline StationsID & $\begin{array}{l}\text { Foreign key to Stations point feature } \\
\text { class }\end{array}$ & $\begin{array}{l}\text { Multiple observations made at same locality will likely have } \\
\text { same StationsID value. Null values permitted }\end{array}$ \\
\hline MapUnit & $\begin{array}{l}\text { Records map unit to which this } \\
\text { observation pertains. Foreign key to } \\
\text { DescriptionOfMapUnits table }\end{array}$ & $\begin{array}{l}\text { Values obtained by intersection with feature class MapUnitPolys. } \\
\text { Null values permitted only for point features that lie outside } \\
\text { extent of MapUnitPolys feature class }\end{array}$ \\
\hline LocationSourceID & $\begin{array}{l}\text { Identifies source of location of this point } \\
\text { feature. Foreign key to DataSources table }\end{array}$ & Null values not permitted \\
\hline OrientationSourceID & $\begin{array}{l}\text { Identifies source of orientation data } \\
\text { for this point feature. Foreign key to } \\
\text { DataSources table }\end{array}$ & Null values not permitted \\
\hline Notes & Optional field & $\begin{array}{l}\text { Free text for additional information specific to this point } \\
\text { feature. Null values permitted }\end{array}$ \\
\hline OrientationPoints_ID & Primary key & $\begin{array}{l}\text { Examples of values are "ORP1", "ORP2". Values must be } \\
\text { unique in database. Null values not permitted }\end{array}$ \\
\hline
\end{tabular}


Table 23. Fields in GeochronPoints (an as-needed point feature class in the GeologicMap feature dataset).

[Abbreviations: ${ }^{14} \mathrm{C}$, carbon-14; ka, thousand years before present; K-Ar, potassium-argon; Ma, million years before present; Rb-Sr, rubidium-strontium; RMSE, root mean square error; yr B.P., years before present. Content of fields in magenta type (in this case, the Type, ErrorMeasure, and AgeUnits fields) must be defined in Glossary table. See also, tables 1, 20,21]

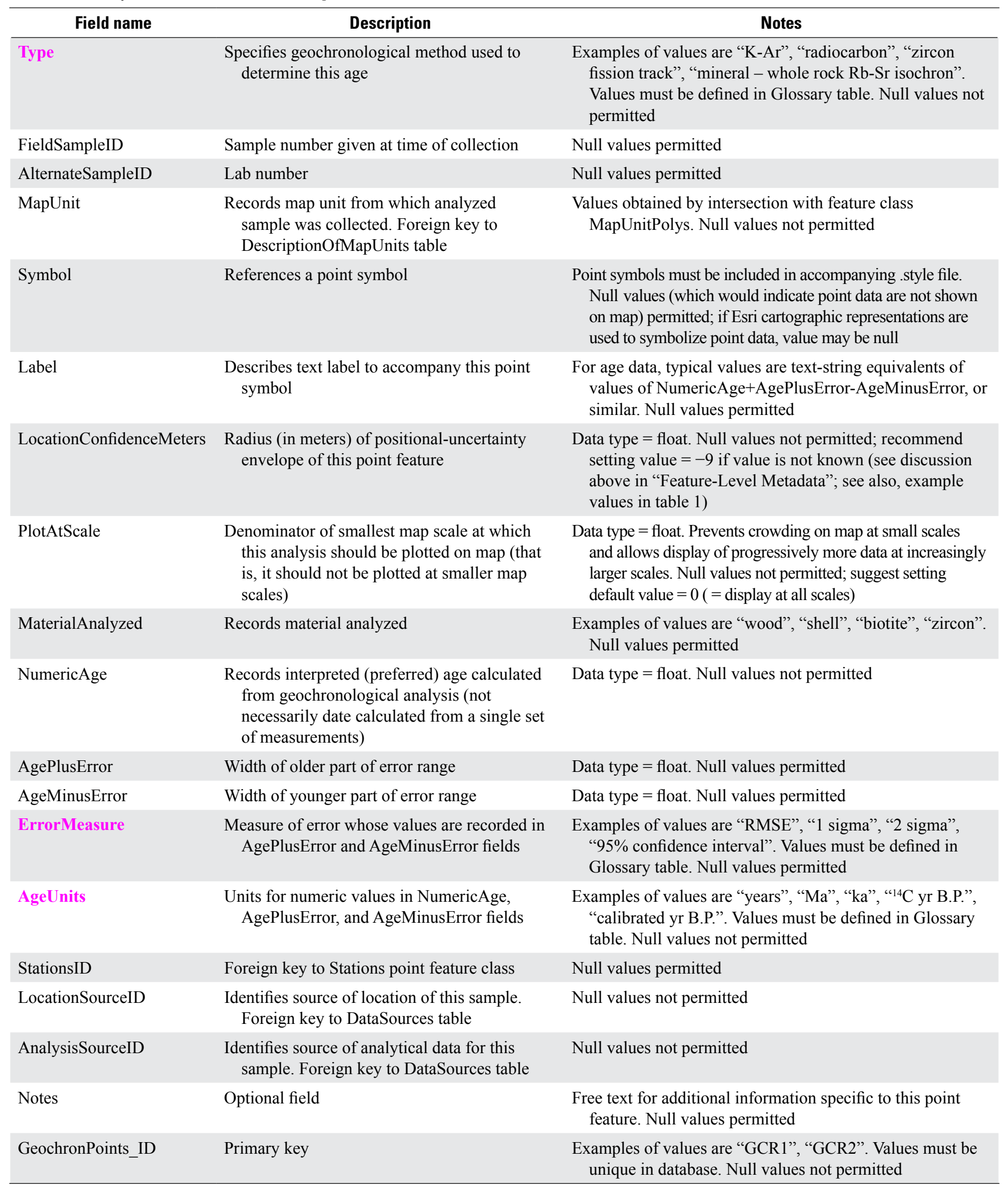


Table 24. Fields in Stations (an as-needed point feature class in the GeologicMap feature dataset).

[See also, tables 20, 21, 25]

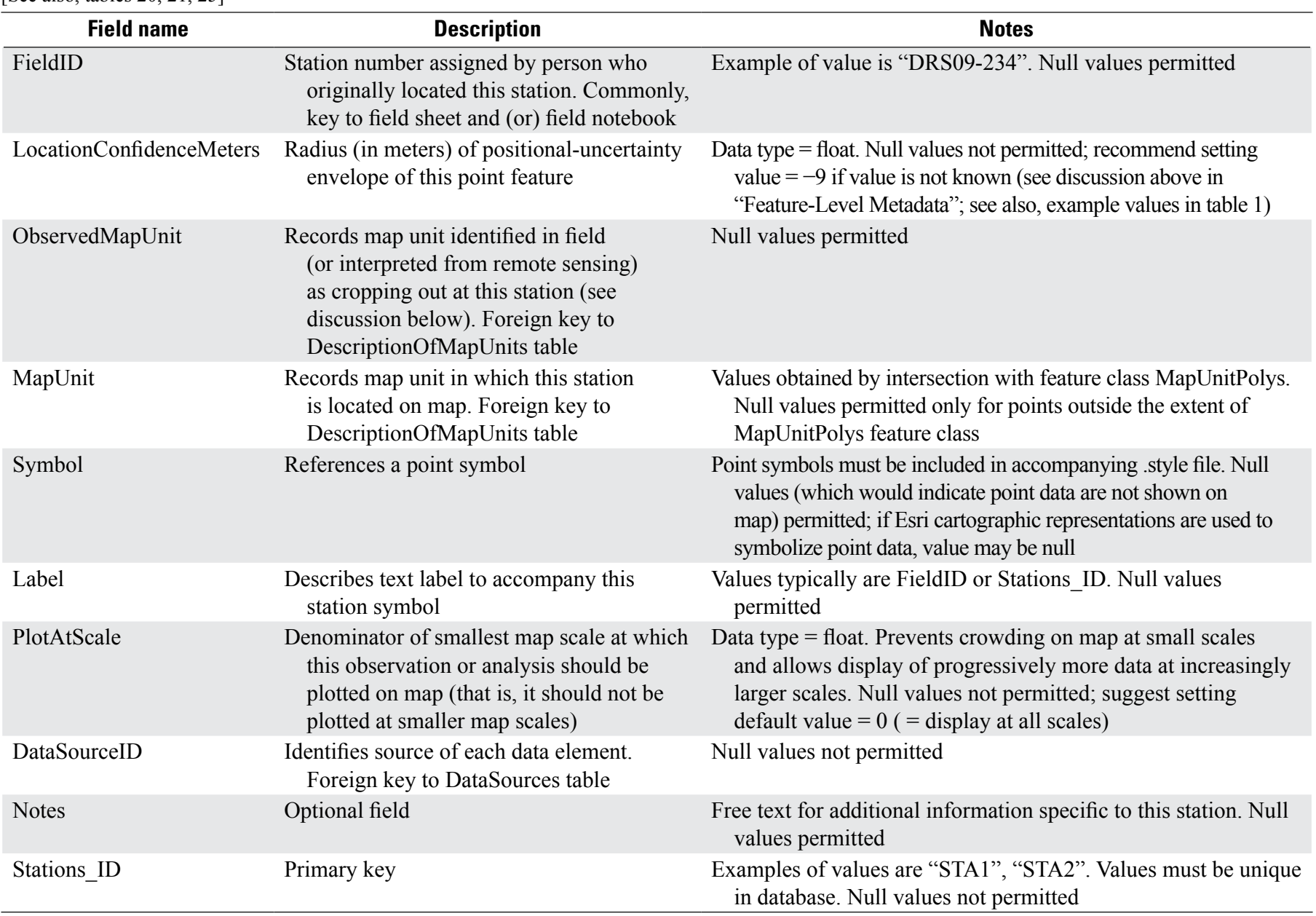

Table 25. Examples of additional fields that might be included in a Stations point feature class.

[Abbreviations: GPS, Global Positioning System; m, meter(s); N, north; W, west. Content of fields in magenta type (in this case, the LocationMethod field) must be defined in Glossary table. See also, tables 20, 21, 24]

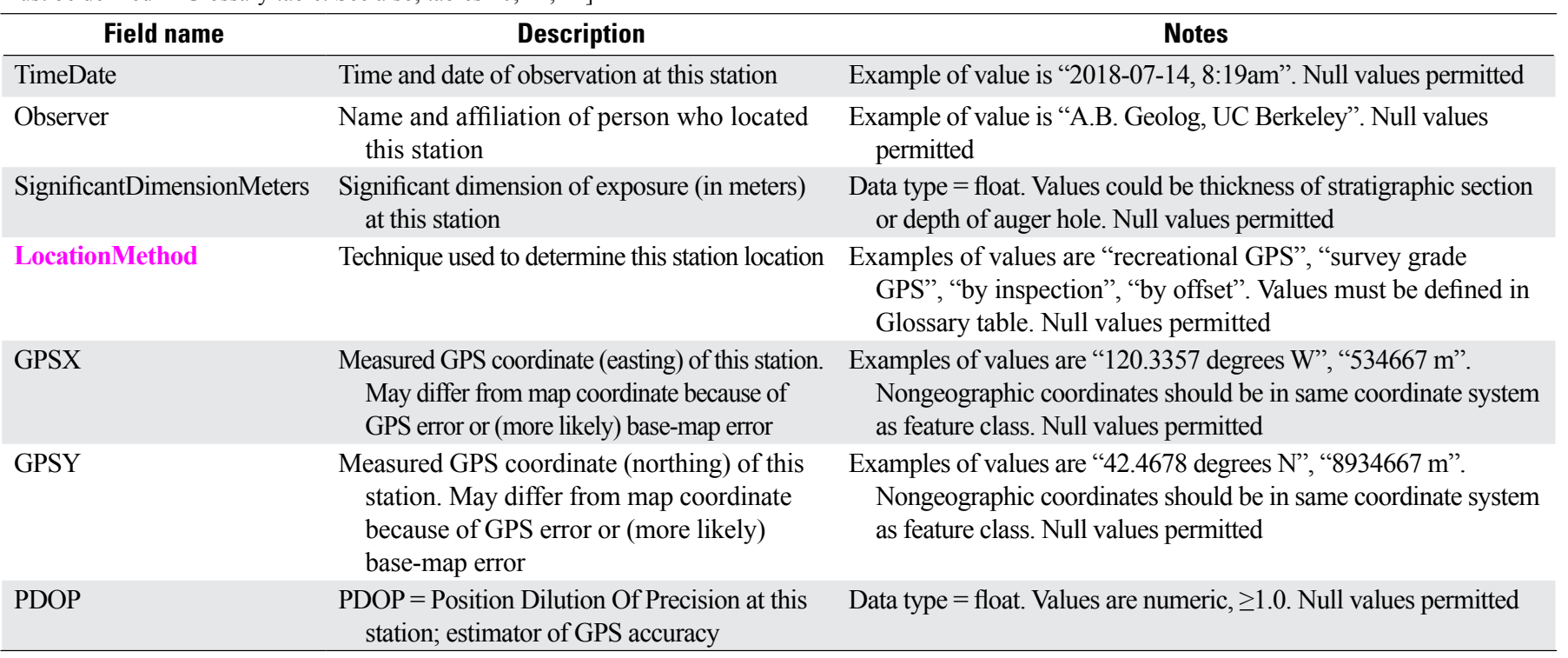


The ObservedMapUnit field is provided because (1) occasionally a station is located in a geologic unit whose exposure is too small to be distinguished from its surrounding map unit at the scale of mapping, (2) a station may be identified in the field as being located in one map unit and later is reinterpreted as being located in another map unit, or (3) a geologic unit that is observed at a station located at depth (for example, in a pit exposure, a borehole, or a bluff) may not be the same as the map unit that is shown on the geologic map at that location.

\section{MapUnitPoints (Point Feature Class)}

Very small, equant or subequant map-unit extents, which are too small to show as polygons at the scale of the map graphic, may be represented as point features. If such point features are present on a map, they should be encoded in the MapUnitPoints feature class. A MapUnitPoints feature class should have an_ID field named MapUnitPoints_ID; other field names, values, and usage should be identical to those shown in table 12, with the addition of the LocationConfidenceMeters, ExistenceConfidence, and PlotAtScale fields (see table 20 for definitions and discussion of these three fields).

\section{Examples of Other As-Needed Feature Classes}

\section{GeologicLines (Line Feature Class)}

Line features such as the traces of dikes, coal seams, ash beds, other kinds of key beds, fault scarps, anticline and syncline hinge-surface traces, and isograd traces commonly are shown on geologic maps. Such features are usefully represented in a single feature class; alternatively, they could be divided thematically into separate feature classes such as FoldLines, FaultScarpLines, KeyBedLines, DikeLines, and IsogradLines. These line features share the following properties:

- They correspond to features that exist within the Earth, and they may be concealed beneath younger, covering material.

- They are likely to be located with an accuracy that can be estimated.

- They do not participate in map-unit topology (that is, they do not delineate map-unit polygons).

Table 26 describes the fields (and their values) that populate the GeologicLines feature class.

As with the ContactsAndFaults feature class, some line features may have the same Type values but be symbolized differently. For example, the line features "anticline", "approximately located anticline", "concealed anticline", and "inferred anticline" would all have a Type value = "anticline" but have different values of IsConcealed, LocationConfidenceMeters, ExistenceConfidence, and (or) IdentityConfidence fields and, thus, might be symbolized differently.
Topology Rules

The following topology rules would apply to the GeologicLines feature class:

- Lines must not self-overlap.

- Lines must not self-intersect.

\section{MapUnitLines (Line Feature Class)}

Very narrow map-unit extents, which are too small to show as polygons at the scale of the map graphic, may be represented as line features. If such line features are present on a map, they should be encoded in the MapUnitLines feature class. A MapUnitLines feature class should have an_ID field named MapUnitLines_ID; other field names, values, and usage should be identical to those shown in table 12, with the addition of LocationConfidenceMeters, ExistenceConfidence, and PlotAtScale fields (see tables 13 and 20 for definitions and discussion of these three fields).

Topology Rules

The following topology rules would apply to the MapUnitLines feature class:

- Lines must not overlap.

- Lines must not intersect.

- Lines must not self-overlap.

\section{CartographicLines (Line Feature Class)}

Some lines on maps (for example, cross-section lines) are cartographic constructs only and have no real-world physical existence, such that values of LocationConfidenceMeters, ExistenceConfidence, and IdentityConfidence attributes are meaningless. Furthermore, they are never shown as concealed beneath a covering unit, and they do not participate in map-unit topology. Table 27 describes the fields (and their values) that populate the CartographicLines feature class.

\section{IsoValueLines (Line Feature Class)}

Lines such as structure contours, concentration isopleths, and hydraulic-head contours share certain properties: (1) they have an associated value (for example, elevation, concentration, hydraulic potential) that is a real number, (2) they have a definable uncertainty in their location, and (3) they describe an idealized surface that is not shown as being concealed beneath covering map units. These lines may be represented in the IsoValueLines feature class described in table 28.

Note that a ValueConfidence field is used here instead of a LocationConfidenceMeters field; either could be used to specify the real-world locational uncertainty, but, in the case of structure contours (or concentration contours), vertical uncertainty is generally more useful than horizontal uncertainty. 
Table 26. Fields in Geologic Lines (an as-needed line feature class in the GeologicMap feature dataset).

[Abbreviations: N, no; Y, yes. Content of fields in magenta type (in this case, the Type, ExistenceConfidence, and IdentityConfidence fields) must be defined in Glossary table]

\begin{tabular}{|c|c|c|}
\hline Field name & Description & Notes \\
\hline Type & $\begin{array}{l}\text { Specifies type of line feature represented by } \\
\text { this database row }\end{array}$ & $\begin{array}{l}\text { Examples of values are "syncline hinge surface trace", } \\
\text { "biotite isograd". Values must be defined in Glossary } \\
\text { table. Null values not permitted }\end{array}$ \\
\hline LocationConfidenceMeters & $\begin{array}{l}\text { Half width (in meters) of positional-uncertainty } \\
\text { envelope around this line feature }\end{array}$ & $\begin{array}{l}\text { Data type = float. Null values not permitted; recommend } \\
\text { setting value }=-9 \text { if value is not known (see discussion } \\
\text { above in "Feature-Level Metadata" and example values } \\
\text { in table 1) }\end{array}$ \\
\hline ExistenceConfidence & $\begin{array}{l}\text { Indicates how confidently existence of this } \\
\text { line feature has been determined }\end{array}$ & $\begin{array}{l}\text { Typical values are "certain", "questionable", "unspecified". } \\
\text { Values must be defined in Glossary table. Null values not } \\
\text { permitted; suggest setting default value = "certain" }\end{array}$ \\
\hline IdentityConfidence & $\begin{array}{l}\text { Indicates how confidently this line feature } \\
\text { has been identified as a particular type }\end{array}$ & $\begin{array}{l}\text { Typical values are "certain", "questionable", "unspecified". } \\
\text { Values must be defined in Glossary table. Null values not } \\
\text { permitted; suggest setting default value = "certain" }\end{array}$ \\
\hline Label & Describes text label for this line feature & $\begin{array}{l}\text { Can be used to store fold name or other easily understood } \\
\text { name for line feature. Null values are typical }\end{array}$ \\
\hline DataSourceID & $\begin{array}{l}\text { Identifies source of each data element. } \\
\text { Foreign key to DataSources table }\end{array}$ & Null values not permitted \\
\hline Notes & Optional field & $\begin{array}{l}\text { Free text for additional information specific to this line } \\
\text { feature. Null values permitted }\end{array}$ \\
\hline
\end{tabular}

Table 27. Fields in CartographicLines (an as-needed line feature class in the GeologicMap feature dataset).

[Content of fields in magenta type (in this case, the Type field) must be defined in Glossary table]

\begin{tabular}{|c|c|c|}
\hline Field name & Description & Notes \\
\hline Type & $\begin{array}{l}\text { Specifies type of cartographic line feature } \\
\text { represented by this database row }\end{array}$ & $\begin{array}{l}\text { Example of value is "cross section line". Values must be } \\
\text { defined in Glossary table. Null values not permitted }\end{array}$ \\
\hline Label & Describes text label for this line feature & $\begin{array}{l}\text { Can be used to store cross-section designation (for example, } \\
\text { "A-A"') or other label for line feature. Null values permitted }\end{array}$ \\
\hline DataSourceID & $\begin{array}{l}\text { Identifies source of each data element. Foreign } \\
\text { key to DataSources table }\end{array}$ & Null values not permitted \\
\hline Notes & Optional field & $\begin{array}{l}\text { Free text for additional information specific to this line feature. } \\
\text { Null values permitted }\end{array}$ \\
\hline CartographicLines_ID & Primary key & $\begin{array}{l}\text { Examples of values are "CAL1", "CAL2". Values must be } \\
\text { unique in database. Null values not permitted }\end{array}$ \\
\hline
\end{tabular}


Table 28. Fields in IsoValueLines (an as-needed line feature class in the GeologicMap feature dataset).

[Abbreviations: As, arsenic; ppm, parts per million; Sr, strontium. Content of fields in magenta type (in this case, the Type field) must be defined in Glossary table]

\begin{tabular}{|c|c|c|}
\hline Field name & Description & Notes \\
\hline Type & $\begin{array}{l}\text { Specifies kind of line feature } \\
\text { represented by this database } \\
\text { row }\end{array}$ & $\begin{array}{l}\text { Examples of values are "top of Big Muddy seam", "ppm Sr", "hydraulic potential } \\
\text { in Stoneyard aquifer". Values must be defined in Glossary table; definition must } \\
\text { contain units for associated Value and ValueConfidence fields; and Value and } \\
\text { ValueConfidence must have same units (for example, for Type = "ppm_As", } \\
\text { values might be Definition = "arsenic concentration in unconfined aquifer; Value } \\
\text { and ValueConfidence are in parts per million; ValueConfidence is estimated } \\
\text { 1-sigma uncertainty"). Null values not permitted }\end{array}$ \\
\hline ValueConfidence & Value uncertainty & $\begin{array}{l}\text { Data type = float. Null values not permitted; recommend setting value }=-9 \text { if } \\
\text { value is not known (see discussion above in "Feature-Level Metadata") }\end{array}$ \\
\hline DataSourceID & $\begin{array}{l}\text { Identifies source of each data } \\
\text { element. Foreign key to } \\
\text { DataSources table }\end{array}$ & Null values not permitted \\
\hline Notes & Optional field & Free text for additional information specific to this feature. Null values permitted \\
\hline IsoValueLines_ID & Primary key & $\begin{array}{l}\text { Examples of values are "IVL1", "IVL2". Values must be unique in database. } \\
\text { Null values not permitted }\end{array}$ \\
\hline
\end{tabular}

\section{Overlay Polygons (the MapUnitOverlayPolys and OverlayPolys Polygon Feature Classes)}

Geologic maps sometimes show, in addition to the map-unit polygons, areas of overlying material(s) or some other aspect of earth materials or character (for example, a dike swarm or an alteration zone); such areas may lie entirely within a map-unit polygon, or they may cross over several polygons. On the map graphic, such overlying material commonly is shown by an overlay pattern (for example, diagonal lines or scattered red dots) overprinted onto the map-unit color; the edges of such overlays usually are shown by a scratch boundary (that is, without a bounding line).

These overlay polygons are not part of the set of map-unit polygons that cover the map area without gaps or overlaps; thus, they do not belong in the feature class MapUnitPolys. The topological relations among these overlay polygons are likely to be complicated (for example, alteration-zone boundaries usually do not coincide with bedrock-map-unit boundaries, but they can coincide with some faults and with unconsolidated-deposit boundaries). And so, they are not easily prescribed by a simple set of rules; however, they usually fall into one of the following two general categories:

- Some overlay polygons are described in the DMU along with the map-unit polygons. For example, in Booth and others (2009), the first four units listed in the DMU (modified land, m; graded areas, gr; artificial fill, af; and landfill debris, afl) are shown as overlay polygons.
In the GeMS schema, such overlay polygons should be described in the feature class MapUnitOverlayPolys, in which the MapUnit field references an entry in the DescriptionOfMapUnits table.

- Some overlay polygons are described in the Explanation of Map Symbols along with other symbols such as contacts and bedding attitudes. For example, in Castor and others (2013), phyllosilicate alteration is shown as a stipple (dot) overlay that is identified in the symbol explanation with the contacts, faults, various vein types, and strike and dip symbols. Unlike modified land in Booth and others (2009), phyllosilicate alteration is not treated as a map unit in Castor and others (2013). In the GeMS schema, such overlay polygons should be described in the feature class OverlayPolys, in which the Type field references an entry in the Glossary table.

If some types of overlay polygons on a map have additional attributes, it may be useful to divide these into multiple feature classes, in which the division is based on the necessary attribute structure. Tables 29 and 30 describe the fields (and their values) that populate the MapUnitOverlayPolys feature class and the OverlayPolys feature class, respectively.

\section{Topology Rules}

No topology rules are prescribed for either type of overlay-polygon feature class. 
Table 29. Fields in MapUnitOverlayPolys (an as-needed polygon feature class in the GeologicMap feature dataset).

[Content of fields in magenta type (in this case, the IdentityConfidence field) must be defined in Glossary table]

\begin{tabular}{|c|c|c|}
\hline Field name & Description & Notes \\
\hline MapUnit & $\begin{array}{l}\text { Short, easily understood ASCII-character }{ }^{1} \text { identifier } \\
\text { for map unit represented by this overlay polygon. } \\
\text { Foreign key to DescriptionOfMapUnits table }\end{array}$ & $\begin{array}{l}\text { Examples of values are "m", "col". Values must be defined in } \\
\text { DescriptionOfMapUnits table. Null values not permitted }\end{array}$ \\
\hline Symbol & References an area-fill symbol & $\begin{array}{l}\text { Area-fill symbols (map-unit color, pattern, or color+pattern) } \\
\text { must be included in accompanying .style file. Null values } \\
\text { permitted. May or may not be same as Symbol value } \\
\text { defined for MapUnit in DescriptionOfMapUnits table }\end{array}$ \\
\hline Notes & Optional field & $\begin{array}{l}\text { Free text for additional information specific to this overlay } \\
\text { polygon. Null values permitted }\end{array}$ \\
\hline MapUnitOverlayPolys_ID & Primary key & $\begin{array}{l}\text { Examples of values are "MUOP1", "MUOP2". Values } \\
\text { must be unique in database. Null values not permitted }\end{array}$ \\
\hline
\end{tabular}

${ }^{1}$ ASCII, American Standard Code for Information Interchange; ASCII characters mainly consist of lower and upper case letters, numerals, and certain punctuation characters (specifically, ones that can be accessed using the Shift key).

Table 30. Fields in OverlayPolys (an as-needed polygon feature class in the GeologicMap feature dataset).

[Content of fields in magenta type (in this case, the Type and IdentityConfidence fields) must be defined in Glossary table]

\begin{tabular}{|c|c|c|}
\hline Field name & Description & Notes \\
\hline Type & $\begin{array}{l}\text { Specifies type of polygon feature } \\
\text { represented by this database row }\end{array}$ & $\begin{array}{l}\text { Example of value is "phyllosilicate alteration". Values must be defined } \\
\text { in Glossary table. Null values not permitted }\end{array}$ \\
\hline IdentityConfidence & $\begin{array}{l}\text { Indicates how confidently this overlay } \\
\text { polygon has been identified as a } \\
\text { particular type }\end{array}$ & $\begin{array}{l}\text { Typical values are "certain", "questionable", "unspecified". Values } \\
\text { must be defined in Glossary table. Null values not permitted; suggest } \\
\text { setting default value = "certain" }\end{array}$ \\
\hline Label & $\begin{array}{l}\text { Describes text label for this overlay } \\
\text { polygon. Field from which map label } \\
\text { (if needed) is generated }\end{array}$ & $\begin{array}{l}\text { May or may not be same as Type value. Null values permitted (as } \\
\text { overlay polygons usually are not labeled, null values are common) }\end{array}$ \\
\hline Symbol & References an area-fill symbol & $\begin{array}{l}\text { Area-fill symbols (color, pattern, or color+pattern) must be included in } \\
\text { accompanying .style file. Null values permitted }\end{array}$ \\
\hline DataSourceID & $\begin{array}{l}\text { Identifies source of each data element. } \\
\text { Foreign key to DataSources table }\end{array}$ & Null values not permitted \\
\hline Notes & Optional field & $\begin{array}{l}\text { Free text for additional information specific to this overlay polygon. } \\
\text { Null values permitted }\end{array}$ \\
\hline
\end{tabular}

\section{DataSourcePolys (Polygon Feature Class)}

For many geologic maps, it is useful or necessary to provide an index map containing polygons that identify the sources of data and interpretations for various parts of the map. Examples of such sources are a previously published map, new mapping, mapping by one of several authors, and mapping using a certain technique (for example, "compiled by A.N. Author (2006) from 1:40,000-scale aerial photographs"). For a map that has only one data source (for example, all new mapping), this feature class would contain one polygon that encompasses the map area. Table 31 describes the fields (and their values) that might populate the DataSourcePolys feature class. 
Table 31. Fields in DataSourcePolys (an as-needed polygon feature class in the GeologicMap feature dataset).

\begin{tabular}{lll}
\hline \multicolumn{1}{c}{ Field name } & \multicolumn{1}{c}{ Description } & Notes \\
\hline DataSourceID & $\begin{array}{c}\text { Identifies source or provenance of data } \\
\text { elements in this polygon. Foreign key to } \\
\text { DataSources table }\end{array}$ & Null values not permitted \\
Notes & Optional field & $\begin{array}{c}\text { Free text for additional information specific to this polygon. } \\
\text { Null values permitted }\end{array}$ \\
DataSourcePolys_ID & Primary key & $\begin{array}{c}\text { Examples of values are "DSP1", "DSP2". Values must be } \\
\text { unique in database. Null values not permitted }\end{array}$ \\
\hline
\end{tabular}

\section{Topology Rules}

The following topology rules would apply to a DataSourcePolys polygon feature class:

- Polygons may overlap.

- All parts of the map area should be encompassed by at least one polygon (no gaps).

\section{Symbolization}

Symbolization is a critical aspect of a geologic map. Not only does it illustrate the geologist's interpretations, it also may depict (by means of color, type size, or other graphical elements) subtleties of interpretation and (or) emphasis that otherwise may not be obvious in the database. Creating adequate symbolization of a geologic map database can be a significant amount of work, but providing an acceptable set of symbols (and symbolization instructions) is of significant value to database users. For these reasons, we require that geologic-map databases include symbols and symbolization instructions to achieve a preferred visualization of the map.

In GeMS, symbolization consists of (1) values of Symbol for all features (use null values where a database feature is not symbolized on the map), (2) an ArcGIS .style file that contains the symbols (for areas, lines, and points) used on the preferred visualization, and (3) an Esri map-composition (.mxd) file. Alternatively, authors may choose to use Esri's cartographic representations to symbolize one or more of the map's layers, in which case a .style file may not be needed.

Generally speaking (and to the extent that it is possible), line and point symbolization should follow the FGDC Digital Cartographic Standard for Geologic Map Symbolization (FGDC, 2006). Most of the symbols in the FGDC cartographic standard have been implemented as an ArcGIS .style file ("FGDC_ GSC_20100414.style") by members of the Geological Survey of Canada; this .style file and its associated font files are available under the Resources heading at the GeMS website (https://ngmdb. usgs.gov/Info/standards/GeMS/). Note that both the .style file and all its associated font files need to be installed for the .style file to function correctly. Note also that, to use this .style file, it is necessary to left-pad the original FGDC symbol identifiers with zeroes so that each part of the identifier has a two- or threecharacter width (for example, symbol 1.1.3 becomes 01.01.03, and symbol 1.1.25 becomes 01.01.25).

Although not a requirement, CMYK color fills for map-unit polygons may be selected from the FGDC cartographic standard's CMYK Color Chart (FGDC, 2006). An ArcGIS .style file ("FGDCcmyk.style") of this chart is available under the Resources heading at the GeMS website (https://ngmdb.usgs.gov/Info/ standards/GeMS/).

Also not a requirement, geologic age symbol characters are best displayed using the FGDCGeoAge font (U.S. Geological Survey, 2006; available at https://pubs.usgs.gov/tm/2006/11A02/). The OpenType version ("FGDCGeoAge.otf") of this font is recommended; it is available under the Resources heading at the GeMS website (https://ngmdb.usgs.gov/Info/standards/GeMS/).

At this time (2020), a subset of the FGDC cartographic standard's library of symbols also is available as cartographic representations through Esri's Geologic Mapping Template ("GMT") (available at https://www.arcgis.com/home/item.html?id=bb02aa 75305f40ff87fb6106aa297da9; see https://ngmdb.usgs.gov/Info/ standards/GeMS/ for updated links and information). Esri's GMT stores the symbols in feature classes that have been organized according to the sections in the FGDC cartographic standard. Note, however, that this method of organization is not compliant with GeMS, primarily because it considers contacts and faults to be separate feature classes.

If the FGDC cartographic standard does not define a suitable symbol for a particular feature on a map, the standard may be supplemented with either customized symbols or with FGDC symbols that have been repurposed for the map. Such repurposed symbols need to be identified in the RepurposedSymbols table, which is required if FGDC symbols have been repurposed.

For the convenience of users who do not have access to an ArcGIS license, we also suggest that an ArcReader document (.pmf file) be provided (see table 4), along with descriptions of the symbolization (in other words, for map unit areas, populate the AreaFillRGB and AreaFillPatternDescription fields in the DescriptionOfMapUnits table with appropriate values), in order for symbolization to be replicated in other GIS programs or in desktop publishing software. 
Table 32. Fields in RepurposedSymbols (an as-needed nonspatial table).

[Abbreviation: FGDC, Federal Geographic Data Committee]

\begin{tabular}{|c|c|c|}
\hline Field name & Description & Notes \\
\hline FgdcIdentifier & $\begin{array}{l}\text { Zero-padded identifier string (derived } \\
\text { from identifier in FGDC cartographic } \\
\text { standard) }\end{array}$ & $\begin{array}{l}\text { Example of value is "01.01.03" (original FGDC identifier was } \\
\text { "1.1.3"). Null values not permitted }\end{array}$ \\
\hline OldExplanation & $\begin{array}{l}\text { Original symbol description from FGDC } \\
\text { cartographic standard }\end{array}$ & $\begin{array}{l}\text { Example of value is "contact--identity and existence certain, } \\
\text { location approximate". Null values not permitted }\end{array}$ \\
\hline NewExplanation & Symbol usage as repurposed on this map & $\begin{array}{l}\text { Example of value is "limit of tephra deposits from Holocene } \\
\text { eruptions of Glacier Peak". Null values not permitted }\end{array}$ \\
\hline RepurposedSymbol_ID & Primary key & $\begin{array}{l}\text { Examples of values are "RSY1", "RSY2". Values must be unique } \\
\text { in database. Null values not permitted }\end{array}$ \\
\hline
\end{tabular}

\section{RepurposedSymbols (Nonspatial Table)}

As stated above, line and point symbolization should generally follow the FGDC Digital Cartographic Standard for Geologic Map Symbolization (FGDC, 2006) to the greatest extent possible. However, if the FGDC cartographic standard does not include a symbol that is suitable for a particular feature on the geologic map, the standard may be supplemented with customized symbols or with FGDC symbols that have been repurposed for the map. Such repurposed symbols should be identified in the RepurposedSymbols table. Table 32 describes the fields (and their values) that populate the RepurposedSymbols table.

\section{Shapefile Version of the Database}

To make database content available without the requirement of having an ArcGIS license, now or in the future, an open shapefile version of the database is required. This version uses the well-documented shapefile and .dbf formats, which have length limitations of fields (must have $\leq 255$ characters) and field names (must be $\leq 10$ characters).

The open shapefile version should include the following elements:

- A shapefile for each feature class.

- A .dbf file for each nonspatial table.

- For each feature class or nonspatial table that originally contained long ( $>255$ characters) fields, a text file that documents the original contents of the long (now shortened) fields.

- A text file that documents (1) the arrangement of feature classes into feature datasets, (2) the translation of feature class names to shapefile names, (3) the translation of nonspatial table names to .dbf file names, and (4) the translation of long field names to shortened field names.
The script GeMS_TranslateToShape_Arc10.py or its replacement (available at https://github.com/usgs/GeMS Tools) automates the creation of this open shapefile version. The script also creates a simple shapefile version of the database that has truncated content and no related tables (that is, no Glossary, DescriptionOfMapUnits, DataSources, or GeoMaterialDict tables).

\section{References Cited}

Booth, D.B., Troost, K.G., and Schimel, S.A., 2009, Geologic map of northeastern Seattle (part of the Seattle North $7.5^{\prime} \times 15^{\prime}$ quadrangle), King County, Washington: U.S. Geological Survey Scientific Investigations Map 3065, scale 1:24,000, https://pubs.usgs.gov/sim/3065/.

Castor, S.B., House, P.K., Hudson, D.M., and Henry, C.D., 2013, Geologic map of the Flowery Peak quadrangle, Storey and Lyon counties, Nevada: Nevada Bureau of Mines and Geology Map 180, 24 p., scale 1:24,000, https:// pubs.nbmg.unr.edu/Geol-Flowery-Peak-map-only-p/m180a. htm.

Dohar, V., 2004, Migrating from ArcInfo Workstation to ArcGIS, in Soller, D.R., ed., Digital Mapping Techniques '04-Workshop Proceedings: U.S. Geological Survey Open-File Report 2004-1451, p. 33-40, https://pubs.usgs. gov/of/2004/1451/pdf/dohar.pdf.

Everett, D., and Dohar, V., 2012, Integrating style files and carto representation into the geological map flow process (the GSC's implementation of the FGDC geologic symbology), in Soller, D.R., ed., Digital Mapping Techniques '10-Workshop Proceedings: U.S. Geological Survey Open-File Report 2012-1171, p. 93-99, https:// pubs.usgs.gov/of/2012/1171/pdf/usgs_of2012-1171Everett_p93-100.pdf. 
Federal Geographic Data Committee [FGDC] [prepared for the Federal Geographic Data Committee by the U.S. Geological Survey], 2006, FGDC Digital Cartographic Standard for Geologic Map Symbolization: Reston, Va., Federal Geographic Data Committee Document Number FGDC-STD-013-2006, 290 p., 2 plates, https://ngmdb.usgs.gov/fgdc_gds/geolsymstd.php.

Garrity, C.P., and Soller, D.R., 2009, Database of the Geologic Map of North America-Adapted from the map by J.C. Reed, Jr. and others (2005): U.S. Geological Survey Data Series 424, https:// pubs.usgs.gov/ds/424.

Hansen, W.R., ed., 1991, Suggestions to Authors of the Reports of the United States Geological Survey, Seventh edition: Washington, D.C., U.S. Government Printing Office, 289 p.

Neuendorf, K.K.E., Mehl, J.P., Jr., and Jackson, J.A., eds., 2011, Glossary of geology (5th ed., revised): Alexandria, Va., American Geosciences Institute, $779 \mathrm{p}$.

North American Geologic Map Data Model Steering Committee Science Language Technical Team, 2004, Report on progress to develop a North American science-language standard for digital geologic-map databases, in Soller, D.R., ed., Digital Mapping Techniques '04 — Workshop Proceedings: U.S. Geological Survey Open-File Report 2004-1451, p. 85-94, 4 appendixes, https://pubs.usgs.gov/of/2004/1451/nadm/.

Powell, J.W., 1888, Methods of geologic cartography in use by the United States Geological Survey, in Congrès Géologique International, Compte Rendu de la 3me Session, Berlin, 1885: Berlin, Germany, A.W. Schade's Buchdruckerei, p. 221-240.
Reed, J.C., Jr., Wheeler, J.O., and Tucholke, J.E., comps., 2005, Geologic map of North America: Boulder, Colo., Geological Society of America, Decade of North American Geology Continental Scale Map 001, scale 1:5,000,000, https://rock. geosociety.org/Store/detail.aspx?id=DNAGCSMS1.

Soller, D.R., 2009, A classification of geologic materials for web display of national and regional-scale mapping, in Soller, D.R., ed., Digital Mapping Techniques '08Workshop Proceedings: U.S. Geological Survey Open-File Report 2009-1298, p. 105-121, https://pubs.usgs.gov/ of/2009/1298/pdf/usgs_of2009-1298_soller4.pdf.

U.S. Geological Survey, 2006, FGDC Digital Cartographic Standard for Geologic Map Symbolization (PostScript Implementation): U.S. Geological Survey Techniques and Methods, Book 11, Chap. A2, https://pubs.usgs.gov/ tm/2006/11A02/.

U.S. Geological Survey, [in press], Suggestions to Authors of the Reports of the United States Geological Survey, Eighth edition: Reston, Va., U.S. Geological Survey [online-only book and website].

U.S. Geological Survey National Cooperative Geologic Mapping Program [USGS NCGMP], 2010, NCGMP09-Draft standard format for digital publication of geologic maps, version 1.1, in Soller, D.R., ed., Digital Mapping Techniques '09-Workshop Proceedings: U.S. Geological Survey Open-File Report 2010-1335, p. 93-146, 4 appendixes, https://pubs.usgs.gov/ of/2010/1335/pdf/usgs_of2010-1335_NCGMP09.pdf. 


\section{Appendixes}




\section{Appendix 1. Terms and Definitions for the GeoMaterial and GeoMaterialConfidence Fields}

Much of the benefit from a defined database schema depends on use of clearly defined vocabularies. On the basis of many discussions within the geoscience community over the past three decades, it seems to be the consensus (and a logical conclusion) that users of geologic map databases are best served if certain terminology, particularly for lithology, is consistent from one database to another. Such a list of terms commonly is referred to as a controlled-term list or a vocabulary.

The terms that populate the GeoMaterial and GeoMaterialConfidence fields in the DescriptionOfMapUnits table constitute such a controlled-term list. These terms are defined below, and they are encapsulated in a database table (see table 17) generated by the script GeMS_CreateDatabase_Arc10.py (or its replacement), available at the GeMS Toolkit site on GitHub (https://github.com/ usgs/GeMS_Tools). The table is also available as an Excel spreadsheet under the Resources heading at the GeMS website (https:// ngmdb.usgs.gov/Info/standards/GeMS/); the spreadsheet also includes the field HierarchyKey to facilitate sorting.

\section{Scope and Intent}

This classification of materials is intended to characterize a map unit by means of a generalized category (GeoMaterial) based on lithologic and genetic criteria; it applies to the map unit as a whole. The purposes of this classification are to provide a basis for quickly integrating map data from different sources and to convey to the public a simple, general sense of each map unit's lithology and genesis. We recognize that such a classification cannot completely address the immense variety of map units present across the Nation, and we expect that other regionally specific classifications of map units will be developed that are more appropriate to local conditions. The appropriateness of a selected GeoMaterial term for describing a map unit is specified by values in the GeoMaterialConfidence field (see table 1-1), which provide the map user with a potentially useful qualifier term (please refer to the Geologic Materials discussion in the "Design Considerations" section, above).

\section{GeoMaterial Terms}

The lithologic terms in the GeoMaterial field and their definitions are provided below, and their hierarchy is represented by an indented format for clarity. Documentation of this classification, including rationale for its development, was provided in Soller (2009); some terms and definitions in that classification were updated for version 1.1 of GeneralLithology (see "Archive of Previous Versions and Related Information" section at https://ngmdb.usgs.gov/Info/standards/GeMS/). In the nine years of test implementation among the state geological surveys and USGS, this classification was slightly modified for GeMS, and renamed as "GeoMaterial". The current version of this classification is maintained at the GeMS website.

The following is GeMS' vocabulary of GeoMaterial terms (shown in bold type) and their definitions:

- Sedimentary material - An aggregation of particles deposited by gravity, air, water, or ice, or as accumulated by other natural agents operating at Earth's surface such as chemical precipitation or secretion by organisms. May include unconsolidated material (sediment) and (or) sedimentary rock. Does not include sedimentary material directly deposited as a result of volcanic activity.

- Sediment - Unconsolidated material (sediment) composed of particles deposited by gravity, air, water, or ice, or as accumulated by other natural agents operating at Earth's surface such as chemical precipitation or secretion by organisms. Does not include sedimentary material directly deposited as a result of volcanic activity.

- Clastic sediment - Sediment formed by weathering and erosion of preexisting rocks or minerals; eroded particles or "clasts" are transported and deposited by gravity, air, water, or ice.

- Sand and gravel of unspecified origin - Sediment composed mostly of sand and (or) gravel, formed by weathering and erosion of preexisting rocks or minerals; eroded particles or "clasts" are transported and deposited by gravity, air, water, or ice.

- Silt and clay of unspecified origin - Sediment composed mostly of silt and (or) clay, formed by weathering and erosion of preexisting rocks or minerals; eroded particles or "clasts" are transported and deposited by gravity, air, water, or ice.

- Alluvial sediment - Unconsolidated material deposited by streams or other bodies of running water as sorted or semisorted sediment in streambed, or on its floodplain or delta, or as cone or fan at base of mountain slope. Grain size varies from clay to gravel. 
Table 1-1. Values (and their definitions) that populate the GeoMaterialConfidence field in a DescriptionOfMapUnits table.

[Note that, when creating a new database, the script GeMS_CreateDatabase_Arc10.py (available at https://github.com/usgs/GeMS_Tools) or its replacement inserts these GeoMaterialConfidence terms and definitions into Glossary table. See also, table 14]

\section{Term Definition}

\begin{tabular}{l} 
High The selected term in the GeoMaterial field (and its definition) adequately characterizes ${ }^{1}$ the overall lithologic nature of rocks \\
and (or) sediments in the map unit \\
Medium $\quad \begin{array}{l}\text { The selected term in the GeoMaterial field (and its definition) generally characterizes the overall lithologic nature of rocks } \\
\text { and (or) sediments in the map unit, but one or more significant but minor lithologies are not adequately described by the } \\
\text { selected term } \\
\text { Either (1) the overall lithologic nature of rocks and (or) sediments in this map unit is not adequately classifiable using the } \\
\text { available list of GeoMaterial terms (and their definitions), but the selected term is the best available, or (2) this map unit is } \\
\text { not sufficiently known enough to confidently assign a GeoMaterial term }\end{array}$ \\
\hline
\end{tabular}

${ }^{1}$ Regarding the subjective term "adequately characterizes", please refer to the context and objectives of this classification as described above in the "Scope and Intent" section of this appendix.

- Alluvial sediment, mostly coarse-grained - Unconsolidated material deposited by streams or other bodies of running water as sorted or semisorted sediment in streambed, or on its floodplain or delta, or as cone or fan at base of mountain slope. Sediment is mostly sand, gravel, and coarser material but may also contain some silt and clay.

- Alluvial sediment, mostly fine-grained - Unconsolidated material deposited by streams or other bodies of running water as sorted or semisorted sediment in streambed, or on its floodplain or delta, or as cone or fan at base of mountain slope. Sediment is mostly silt and clay but may also contain some sand and gravel.

- Glacial till - Mostly unsorted and unstratified material, generally unconsolidated, deposited directly by and underneath or adjacent to glacier without subsequent reworking by meltwater. Consists of heterogeneous mixture of clay, silt, sand, gravel, and boulders, ranging widely in size and shape.

- Glacial till, mostly sandy - Mostly unsorted and unstratified material, generally unconsolidated, deposited directly by and underneath or adjacent to glacier without subsequent reworking by meltwater. Consists of heterogeneous mixture of clay, silt, sand, gravel, and boulders, ranging widely in size and shape. Relatively sandy in texture.

- Glacial till, mostly silty - Mostly unsorted and unstratified material, generally unconsolidated, deposited directly by and underneath or adjacent to glacier without subsequent reworking by meltwater. Consists of heterogeneous mixture of clay, silt, sand, gravel, and boulders, ranging widely in size and shape. Relatively loamy (silty) in texture.

- Glacial till, mostly clayey - Mostly unsorted and unstratified material, generally unconsolidated, deposited directly by and underneath or adjacent to glacier without subsequent reworking by meltwater. Consists of heterogeneous mixture of clay, silt, sand, gravel, and boulders, ranging widely in size and shape. Relatively clayey in texture.

- Ice-contact and ice-marginal sediment - Mostly sand-, silt-, and gravel-sized particles or "clasts" derived from rock or preexisting sediment that has been eroded and transported by glaciers. As glacier melted, material was deposited by running water essentially in contact with glacial ice or was transported and deposited by glacially fed streams. Includes sediment deposited into water bodies adjacent to glacier.

- Ice-contact and ice-marginal sediment, mostly coarse-grained - Mostly sand- and gravel-sized particles or "clasts", with lesser amounts of silt and clay, derived from rock or preexisting sediment that has been eroded and transported by glaciers. As glacier melted, material was deposited by running water essentially in contact with glacial ice or was transported and deposited by glacially fed streams. Includes sediment deposited into water bodies adjacent to glacier.

- Ice-contact and ice-marginal sediment, mostly fine-grained - Mostly silt- and clay-sized particles or "clasts", with lesser amounts of sand and gravel, derived from rock or preexisting sediment that has been eroded and transported by glaciers. As glacier melted, material was deposited by running water essentially in 
contact with glacial ice or was transported and deposited by glacially fed streams. Includes sediment deposited into water bodies adjacent to glacier.

- Eolian sediment - Silt- and sand-sized sediment, deposited by wind.

- Dune sand - Mostly sand-sized sediment, deposited by wind. Typically characterized by various dune landforms.

- Loess - Silty sediment, deposited by wind, commonly near glacial margin.

- Lacustrine sediment - Mostly well-sorted and well-bedded material that ranges in grain size from clay to gravel, deposited in perennial to intermittent lakes. Much of sediment is derived from material eroded and transported by streams. Includes deposits of lake-marginal beaches and deltas.

- Lacustrine sediment, mostly coarse-grained - Mostly well-sorted and well-bedded material, generally sand and gravel sized, with lesser amounts of silt and clay, deposited in perennial to intermittent lakes. Much of sediment is derived from material eroded and transported by streams. Mostly deposits of lake-marginal beaches and deltas.

- Lacustrine sediment, mostly fine-grained - Mostly well-sorted and well-bedded material, generally silt and clay sized, with lesser amounts of sand, deposited in perennial to intermittent lakes.

- Playa sediment - Fine-grained clastic sediment and evaporitic salts, deposited in ephemeral lakes in centers of undrained basins. Includes material deposited in playas, mud flats, salt flats, and adjacent saline marshes. Generally interbedded with eolian sand and with lacustrine sediment deposited during wetter climatic periods; commonly intertongues upslope with sediment deposited by alluvial fans.

- Coastal zone sediment - Mud and sand, with lesser amounts of gravel, deposited on beaches, on barrier islands, or in nearshore-marine, deltaic, or various low-energy shoreline (mud flat, tidal flat, sabka, algal flat) environments.

- Coastal zone sediment, mostly coarse-grained - Mostly sand, silt, and gravel, deposited on beaches, in dunes, and in shallow-marine and related alluvial environments.

- Coastal zone sediment, mostly fine-grained - Mostly clay and silt, deposited in lagoons, tidal flats, backbarriers, and coastal marshes.

- Marine sediment - Mud and sand, deposited in various marine environments. May originate from erosion of rocks and sediment or may be derived from marine organisms (of carbonate or siliceous composition).

- Marine sediment, mostly coarse-grained - Mud, sand, and gravel eroded from rocks and sediment on land, transported by streams, and deposited in marine deltas and basins. Mostly siliceous in composition.

- Marine sediment, mostly fine-grained - Mostly clay- and silt-sized sediment, deposited in relatively deep, quiet water, far removed from areas where coarser grained clastic sediments are washed into marine environment. Includes sediment derived from marine organisms.

- Mass movement sediment - Sediment formed by downslope transport of particles or "clasts" produced by weathering and breakdown of underlying rock, sediment, and (or) soil. Composed of poorly sorted and poorly stratified material that ranges in size from clay to boulders. Includes colluvial, landslide, talus, and rock-avalanche deposits.

- Colluvium and other widespread mass-movement sediment - Sediment formed by slow, relatively widespread, downslope transport of particles or "clasts" produced by weathering and breakdown of underlying rock, sediment, and (or) soil. Composed of poorly sorted and poorly stratified material that ranges in size from clay to boulders.

- Debris flows, landslides, and other localized mass-movement sediment - Sediment formed by relatively localized, downslope transport of particles or "clasts" produced by weathering and breakdown of underlying rock, sediment, and (or) soil. Composed of poorly sorted and poorly stratified material that ranges in size from clay to boulders. Speed of downslope transport ranges from rapid to imperceptible.

- Residual material - Unconsolidated material, developed in place by weathering of underlying rock or sediment. Usually forms relatively thin surface layer that conceals unweathered or partly altered source material. Material from which soils are formed.

- Carbonate sediment - Sediment formed by biotic or abiotic precipitation from aqueous solution of carbonates of calcium, magnesium, or iron (for example, limestone, dolomite). 
- Peat and muck - Unconsolidated material, principally composed of plant remains, with lesser amounts of fine-grained clastic sediment. Deposited in water-saturated environment such as swamp, marsh, or bog. With lithification, such material becomes coal.

- Sedimentary rock - Consolidated material (rock) composed of particles transported and deposited by gravity, air, water, or ice, or accumulated by other natural agents operating at Earth's surface, such as chemical precipitation or secretion by organisms. Does not here include sedimentary material directly deposited as result of volcanic activity.

- Clastic sedimentary rock - Sedimentary rock, composed predominantly of particles or "clasts" derived by erosion, weathering, or mass-wasting of preexisting rock and deposited by gravity, air, water, or ice.

- Conglomerate - Sedimentary rock, composed predominantly of particles or "clasts" derived by erosion and weathering of preexisting rock; contains more than 30 percent gravel-sized clasts.

- Sandstone - Sedimentary rock, composed predominantly of particles or "clasts" derived by erosion and weathering of preexisting rock; consists mostly of sand-sized particles, with or without fine-grained matrix of silt or clay.

- Mostly sandstone - Mostly sandstone, interbedded with other sedimentary rocks that locally may include conglomerate and finer grained clastic rocks (mudstone), carbonates, and (or) coal.

- Sandstone and mudstone - Sandstone and mudstone (including shale and siltstone), in approximately equal (or unspecified) proportions.

- Mudstone - Sedimentary rock, composed predominantly of particles or "clasts" derived by erosion and weathering of preexisting rock; consists mostly of mud (that is, silt- and clay-sized particles). Includes shale and siltstone.

- Mostly mudstone - Mostly mudstone, interbedded with other sedimentary rocks that locally may include coarser grained clastic rocks (sandstone, conglomerate), carbonates, and (or) coal.

- Carbonate rock - Sedimentary rock, consisting chiefly of carbonate minerals such as limestone or dolomite.

- Limestone - Carbonate sedimentary rock, consisting chiefly of calcite.

- Dolomite - Carbonate sedimentary rock, consisting chiefly of dolomite. Although "dolostone" is the proper analog to "limestone", it has not often been applied to dolomitic units.

- Mostly carbonate rock - Mostly carbonate rock, interbedded with other sedimentary rock types.

- Chert - Sedimentary rock, composed chiefly of microcrystalline or cryptocrystalline quartz.

- Evaporitic rock - Sedimentary rock, composed primarily of minerals produced by evaporation of saline solution. Examples include gypsum, anhydrite, other diverse sulfates, halite (rock salt), primary dolomite, and rocks composed of various nitrates and borates.

- Iron-rich sedimentary rock - Sedimentary rock, in which at least half (by volume) of observed minerals are iron bearing (hematite, magnetite, limonite group minerals, siderite, iron sulfides).

- Coal and lignite - Organic-rich sedimentary rock, formed from compaction and alteration of plant remains. Coal is consolidated, harder, black rock. Lignite is semiconsolidated, brown to black, earthy material that may contain large particles of recognizable plant parts and tends to crack upon drying.

- Sedimentary and extrusive igneous material - Either (1) sedimentary rock and (or) unconsolidated material (sediment) and extrusive igneous material (volcanic rock and [or] sediment) or (2) volcanic rock and (or) sediment and such material after erosion and redeposition.

- Igneous rock - Rock and fragmental material that solidified from molten or partly molten material (magma).

- Extrusive igneous material - Molten material that was erupted onto Earth's surface, fusing into rock or remaining as unconsolidated particles. Includes pyroclastic flows, air-fall tephra, lava flows, and volcanic mass flows.

- Volcaniclastic (fragmental) material - Rock and unconsolidated material consisting of particles or "clasts" that were formed by volcanic explosion or aerial expulsion from volcanic vent.

- Pyroclastic flows - Hot ash, pumice, and rock fragments erupted from volcano or caldera. Material moves downslope commonly in chaotic flows; once deposited, hot fragments may compact under their own weight and weld together. 
- Felsic-composition pyroclastic flows - Hot ash, pumice, and rock fragments erupted from volcano or caldera. Material moves downslope commonly in chaotic flows; once deposited, hot fragments may compact under their own weight and weld together. Because of their high-silica content and resulting high viscosity, parental magmas tend to erupt explosively. Includes rhyolite, dacite, trachyte, latite; rocks are commonly lightcolored.

- Intermediate-composition pyroclastic flows - Hot ash, pumice, and rock fragments erupted from volcano. Material moves downslope commonly in chaotic flows; once deposited, hot fragments may compact under their own weight and weld together. Parental magma commonly erupts from stratovolcanoes as thick lava flows but also can generate strong explosive eruptions to form pyroclastic flows. Includes rocks that are, in color and mineral composition, intermediate between felsic and mafic rocks (for example, andesite).

- Mafic-composition pyroclastic flows - Hot ash, pumice, and rock fragments erupted from volcano. Material moves downslope commonly in chaotic flows; once deposited, hot fragments may compact under their own weight and weld together. Because of their low silica content and resulting low viscosity, parental magmas tend to erupt gently as lava flows rather than more forcefully as pyroclastic flows. Includes basalt; rocks are commonly dark-colored.

- Air-fall tephra - Fragments of volcanic rock and lava, of various sizes, carried into air by explosions and by hot gases in eruption columns or lava fountains; known as tephra. As tephra falls to ground, with increasing distance from volcano, average size of individual rock particles and thickness of resulting deposit decrease. Fine tephra deposited at some distance from volcano is known as volcanic ash.

- Felsic-composition air-fall tephra - Fragments of volcanic rock and lava, of various sizes, carried into air by explosions and by hot gases in eruption columns or lava fountains; known as tephra. As tephra falls to ground, with increasing distance from volcano, average size of individual rock particles and thickness of resulting deposit decrease. Because of their high silica content and resulting high viscosity, felsic-composition magmas tend to erupt explosively, readily forming pumice and volcanic ash. Composed of light-colored rocks (for example, rhyolite, dacite, trachyte, latite).

- Intermediate-composition air-fall tephra - Fragments of volcanic rock and lava, of various sizes, carried into the air by explosions and by hot gases in eruption columns or lava fountains; known as tephra. As tephra falls to ground, with increasing distance from volcano, average size of individual rock particles and thickness of resulting deposit decrease. Parental magma commonly erupts from stratovolcanoes as thick lava flows but also can generate strong explosive eruptions to form pyroclastic flows. Includes rocks that are, in color and mineral composition, intermediate between felsic and mafic rocks (for example, andesite).

- Mafic-composition air-fall tephra - Fragments of volcanic rock and lava, of various sizes, carried into the air by explosions and by hot gases in eruption columns or lava fountains; known as tephra. As tephra falls to ground, with increasing distance from volcano, average size of individual rock particles and thickness of resulting deposit decrease. Because of their low silica content and resulting low viscosity, parental magmas tend to erupt gently as lava flows, and so these deposits are uncommon. Includes basalt; rocks are commonly dark-colored.

- Lava flows - Lateral, surficial outpourings of molten lava from vent or fissure; also, solidified bodies of rock that form when they cool. Composed generally of fine-grained, dark-colored rocks (for example, basalt), which tend to form extensive sheets that have generally low relief, except in vent areas where cinder cones or shield volcanoes may form. Includes basaltic shield volcanoes, which may become very large (for example, Hawaii).

- Felsic-composition lava flows - Lateral, surficial outpourings of molten lava from vent or fissure; also, solidified bodies of rock that form when they cool. Because of their high silica content and resulting high viscosity, parental magmas tend to erupt explosively, and so these deposits are uncommon. Includes fine-grained, lightcolored rock with rhyolitic, dacitic, trachytic, and latitic composition.

- Intermediate-composition lava flows - Lateral, surficial outpourings of molten lava from vent or fissure; also, solidified bodies of rock that form when they cool. Parental magma commonly erupts from stratovolcanoes as thick lava flows. Includes rocks that are, in color and in mineral composition, intermediate between felsic and mafic rocks (for example, andesite).

- Mafic-composition lava flows - Lateral, surficial outpourings of molten lava from vent or fissure; also, solidified bodies of rock that form when they cool. Low-silica parental magmas have low viscosity and tend to form 
extensive sheets that have generally low relief. Includes basaltic shield volcanoes, which may become very large (for example, in Hawaii). Composed of fine-grained, dark rocks, including basaltic.

- Volcanic mass flow - Volcanic deposits formed by mass movement (for example, debris avalanches, debris flows, lahar deposits), in many cases triggered by volcanic eruption. Debris avalanches that occur on volcanoes clearly without eruptive trigger may be classified as sedimentary (for example, as "Debris flows, landslides, and other localized mass-movement sediment").

- Intrusive igneous rock - Rock that solidified from molten or partly molten material (magma) below Earth's surface.

- Coarse-grained intrusive igneous rock - Rock that solidified from molten or partly molten material (magma) at some depth beneath Earth's surface, thereby cooling slowly enough for mineral crystals to grow large enough to be visible to naked eye.

- Coarse-grained, felsic-composition intrusive igneous rock - Rock that solidified from molten or partly molten material (magma) at some depth beneath Earth's surface, thereby cooling slowly enough for mineral crystals to grow large enough to be visible to naked eye. Composed mostly of light-colored minerals (for example, feldspar, quartz). Includes granitic, syenitic, and monzonitic rock.

- Coarse-grained, intermediate-composition intrusive igneous rock - Rock that solidified from molten or partly molten material (magma) at some depth beneath Earth's surface, thereby cooling slowly enough for mineral crystals to grow large enough to be visible to naked eye. Intermediate in color and in mineral composition (between felsic and mafic igneous rock). Includes dioritic rock.

- Coarse-grained, mafic-composition intrusive igneous rock - Rock that solidified from molten or partly molten material (magma) at some depth beneath Earth's surface, thereby cooling slowly enough for mineral crystals to grow large enough to be visible to naked eye. Composed mostly of feldspar and dark-colored minerals. Includes gabbroic rock.

- Ultramafic intrusive igneous rock - Rock that solidified from molten or partly molten material (magma) at some depth beneath Earth's surface, thereby cooling slowly enough for mineral crystals to grow large enough to be visible to naked eye. Composed almost entirely of mafic minerals (for example, hypersthene, augite, olivine).

- Fine-grained intrusive igneous rock - Rock that solidified from molten or partly molten material (magma) at shallow depth beneath Earth's surface, thereby cooling quickly. Generally fine grained but may contain large mineral crystals (phenocrysts). Mostly found as tabular dikes or sills.

- Fine-grained, felsic-composition intrusive igneous rock - Rock that solidified from molten or partly molten material (magma) at shallow depth beneath Earth's surface, thereby cooling quickly. Generally fine grained but may contain large mineral crystals (phenocrysts). Mostly found as tabular dikes or sills. Composed mostly of light-colored minerals. Includes rhyolitic, dacitic, trachytic, and latitic rock.

- Fine-grained, intermediate-composition intrusive igneous rock - Rock that solidified from molten or partly molten material (magma) at shallow depth beneath Earth's surface, thereby cooling quickly. Generally fine grained but may contain large mineral crystals (phenocrysts). Mostly found as tabular dikes or sills. Intermediate in color and in mineral composition (between felsic and mafic igneous rock). Includes andesitic rock.

- Fine-grained, mafic-composition intrusive igneous rock - Rock that solidified from molten or partly molten material (magma) at shallow depth beneath Earth's surface, thereby cooling quickly. Generally fine grained but may contain large mineral crystals (phenocrysts). Mostly found as tabular dikes or sills. Composed mostly of dark-colored minerals. Includes basaltic rock.

- Exotic-composition intrusive igneous rock - Rock that solidified from molten or partly molten material (magma) below Earth's surface that has exotic mineralogical, textural, or field setting characteristics. Typically dark colored with abundant phenocrysts. Includes kimberlite, lamprophyre, lamproite, and foiditic rocks.

- Igneous and metamorphic rock - Consists of coarse-grained intrusive igneous rock and generally medium- to highgrade metamorphic rock.

- Metamorphic rock - Rock derived from preexisting rocks and altered by essentially solid-state mineralogical, chemical, or structural changes, in response to marked changes in temperature, pressure, deformation, and (or) chemical environment, generally at depth in Earth's crust. 
- Regional metamorphic rock, of unspecified origin - Rock derived from preexisting rocks and altered by essentially solid-state mineralogical, chemical, or structural changes, in response to marked regional changes in temperature, pressure, deformation, and (or) chemical environment, generally at depth in Earth's crust. Origin of preexisting rock is mixed (for example, igneous and sedimentary) or is not known.

- Lower-grade metamorphic rock, of unspecified origin - Rock derived from preexisting rocks and altered by essentially solid-state mineralogical, chemical, or structural changes, in response to relatively mild regional changes in temperature, pressure, deformation, and (or) chemical environment, generally at depth in Earth's crust. Origin of preexisting rock is mixed (for example, igneous and sedimentary) or is not known. Includes slate and phyllite.

- Medium and high-grade regional metamorphic rock, of unspecified origin - Rock derived from preexisting rocks and altered by essentially solid-state mineralogical, chemical, or structural changes, in response to relatively intense regional changes in temperature, pressure, deformation, and (or) chemical environment, generally at depth in Earth's crust. Origin of preexisting rock is mixed (for example, igneous and sedimentary) or is not known. Includes amphibolite, granulite, schist, and gneiss.

- Contact-metamorphic rock - Altered rock that originated by local processes of thermal metamorphism, genetically related to intrusion and extrusion of magmas and taking place at or near contact with body of igneous rock. Metamorphic changes are affected by heat and fluids emanating from magma and by some deformation because of emplacement of igneous mass.

- Deformation-related metamorphic rock - Rock derived from preexisting rocks by essentially solid-state mineralogical, chemical, or structural changes in response to strong deformation, commonly in association with marked changes in temperature, pressure, and (or) chemical environment. Generally forms in narrow, planar zones of local deformation (for example, along faults); characterized by foliation or alignment of mineral grains. Includes mylonite and cataclasite.

- Metasedimentary rock - Rock derived from preexisting sedimentary rocks and altered by essentially solid-state mineralogical, chemical, or structural changes, in response to marked changes in temperature, pressure, deformation, and (or) chemical environment, generally at depth in Earth's crust.

- Slate and phyllite, of sedimentary-rock origin - Fine-grained rock derived from preexisting sedimentary rocks and altered by essentially solid-state mineralogical, chemical, or structural changes, in response to marked changes in temperature, pressure, deformation, and (or) chemical environment, generally at depth in Earth's crust. Includes phyllite and slate (compact, fine-grained rock that possesses strong cleavage and, hence, can be split into slabs and thin plates). Mostly formed from fine-grained material such as mudstone.

- Schist and gneiss, of sedimentary-rock origin - Foliated rock derived from preexisting sedimentary rocks by essentially solid-state mineralogical, chemical, or structural changes in response to marked changes in temperature, pressure, deformation, and (or) chemical environment, generally at depth in Earth's crust. Includes schist (characterized by such strong foliation or alignment of minerals that it readily splits into flakes or slabs) and gneiss (characterized by alternating, irregular bands of different mineral composition). Mostly formed from fine-grained material such as mudstone.

- Meta-carbonate rock - Rock derived from preexisting carbonate sedimentary rocks and altered by essentially solid-state mineralogical, chemical, or structural changes, in response to marked changes in temperature, pressure, deformation, and (or) chemical environment, generally at depth in Earth's crust. Characterized by recrystallization of carbonate minerals in source rock. Includes marble (for which preexisting rock was dominantly limestone or other rock composed of calcite), dolomitic marble, meta-dolostone, and meta-dolomite (for which preexisting rock contained appreciable amount of magnesium).

- Quartzite - Rock derived from preexisting quartz-rich sedimentary rocks (commonly sandstone) and altered by essentially solid-state mineralogical, chemical, or structural changes, in response to marked changes in temperature, pressure, shear stress, and (or) chemical environment, generally at depth in Earth's crust.

- Metaigneous rock - Rock derived from preexisting igneous rocks and altered by essentially solid-state, mineralogical, chemical, or structural changes, in response to marked changes in temperature, pressure, shear stress, and (or) chemical environment, generally at depth in Earth's crust.

- Meta-ultramafic rock - Rock derived from preexisting ultramafic rocks by essentially solid-state, mineralogical, chemical, or structural changes, in response to marked changes in temperature, pressure, deformation, and (or) chemical environment, generally at depth in Earth's crust. Composed mostly of magnesium-bearing minerals (for example, serpentine, talc, magnesite). 
- Meta-mafic rock - Rock derived from preexisting mafic rocks by essentially solid-state, mineralogical, chemical, or structural changes, in response to marked changes in temperature, pressure, deformation, and (or) chemical environment, generally at depth in Earth's crust. Composed mostly of iron- and magnesium-bearing, dark-colored and (or) green minerals. Includes greenstone, amphibolite, and metagabbro.

- Meta-felsic and intermediate rock - Rock derived from preexisting felsic and intermediate-composition rocks by essentially solid-state, mineralogical, chemical, or structural changes, in response to marked changes in temperature, pressure, deformation, and (or) chemical environment, generally at depth in Earth's crust. Composed mostly of light-colored minerals; relatively enriched in silica. Includes metagranite, metadiorite, and meta-andesite.

- Meta-volcaniclastic rock - Rock derived from preexisting volcaniclastic rocks by essentially solid-state, mineralogical, chemical, or structural changes, in response to marked changes in temperature, pressure, deformation, and (or) chemical environment, generally at depth in Earth's crust. Composed of deformed but recognizable particles or clasts of volcanic explosive material.

\section{- Other materials:}

- Rock and sediment — Various rocks and sediment, not differentiated.

- Rock - Various rock types, not differentiated.

- "Made" or human-engineered land - Modern, unconsolidated material known to have human-related origin.

- Water or ice

- Unmapped area

\section{GeoMaterialConfidence Terms}

The appropriateness of a selected GeoMaterial term for characterizing a map unit is described by values in the GeoMaterialConfidence field, which provide the map user with a potentially useful qualifier term. Please refer to the "Scope and Intent" section (in the introductory material of this appendix) before assigning confidence values. Table 1-1 describes the terms (and their definitions) that populate the GeoMaterialConfidence field in a DescriptionOfMapUnits table.

\section{Reference Cited in Appendix 1}

Soller, D.R., 2009, A classification of geologic materials for web display of national and regional-scale mapping, in Soller, D.R., ed., Digital Mapping Techniques '08-Workshop Proceedings: U.S. Geological Survey Open-File Report 2009-1298, 216 p., https://pubs.usgs.gov/of/2009/1298/pdf/usgs_of2009-1298_soller4.pdf.

\section{Published and Unpublished Sources for Terms and Definitions (Modified as Needed) in the GeoMaterial Field, as Listed in Appendix 1}

Blatt, H., Tracy, R.J., and Owens, B.E., 2006, Petrology—Igneous, sedimentary, and metamorphic (3d ed.): New York, W.H. Freeman and Company, $530 \mathrm{p}$.

Hyndman, D.W., 1985, Petrology of igneous and metamorphic rocks (2d ed.): New York, McGraw-Hill, Inc., 576 p.

National Geologic Map Database Project, 2007, Science vocabulary to support the National Geologic Map Database projectLithology terms: U.S. Geological Survey unpublished document, $218 \mathrm{p}$.

Neuendorf, K.K.E., Mehl, J.P., Jr., and Jackson, J.A., eds., 2005, Glossary of geology (5th ed.): Alexandria, Va., American Geological Institute, $779 \mathrm{p}$.

North American Geologic Map Data Model Steering Committee Science Language Technical Team, 2004, Report on progress to develop a North American science-language standard for digital geologic-map databases, in Soller, D.R., ed., Digital Mapping Techniques '04—Workshop Proceedings: U.S. Geological Survey Open-File Report 2004-1451, p. 85-94, 4 appendixes, https:// pubs.usgs.gov/of/2004/1451/nadm/. 
Soller, D.R., and Reheis, M.C., compilers, 2004, Surficial materials in the conterminous United States: U.S. Geological Survey Open-File Report 2003-275, scale 1:5,000,000, https://pubs.usgs.gov/of/2003/of03-275/.

U.S. Geological Survey Volcano Hazards Program, 2008, Glossary: U.S. Geological Survey Volcano Hazards Program website, https://volcanoes.usgs.gov/vsc/glossary/. 


\section{Appendix 2. Optional Database Elements}

Cross sections and the Correlation of Map Units diagram in a geologic map report may, if present, be encoded as image files. At present, this is the conventional, and quite reasonable, approach. Alternatively, these elements may be encoded within the database as feature datasets, as described below. Encoding them within the database facilitates matching symbolization to the map graphic, facilitates queries and analysis, and eases reuse of these elements. We also define two optional nonspatial tables (MiscellaneousMapInformation and StandardLithology) below.

\section{Cross Sections (Feature Datasets)}

If cross sections are included in the database, each cross section should be in a separate feature dataset, as each cross section has its own spatial reference framework. A single cross-section feature dataset should be named CrossSectionA, and its feature-class names should include the abbreviation "CSA" (for example, CSAMapUnitPolys, CSAContactsAndFaults, etc.). Additional cross-section feature datasets should be named CrossSectionB, CrossSectionC, and so on (and abbreviated as "CSB", "CSC", etc., in the feature-class names).

Each cross-section feature dataset should have, at a minimum, two feature classes, one for its map units and one for the lines that bound them: for example, CrossSectionA would have CSAMapUnitPolys and CSAContactsAndFaults. The primary-key field for the CSAMapUnitPolys feature class would be CSAMapUnitPolys_ID (values = "CSAMUP1", "CSAMUP2", etc.); for CSAContactsAndFaults, it would be CSAContactsAndFaults_ID (values = "CSACAF1", "CSACAF2", etc.). Data types, usage, and topology rules for these two feature classes are identical to those for ContactsAndFaults and MapUnitPolys.

If other elements are depicted on the cross section, such as point features or certain line features that do not participate in map-unit topology, then the appropriate feature classes (for example, CSAOrientationPoints, CSAGeologicLines) should be created.

\section{Correlation of Map Units (Feature Dataset)}

The Correlation of Map Units (CMU) diagram found on many geologic maps can be encoded as a feature dataset in the database if so desired. The feature dataset should be named CorrelationOfMapUnits, and its feature-class names should include the abbreviation "CMU" (for example, CMUMapUnitPolys, etc.). The CMU dataset should have at least two feature classes (CMUMapUnitPolys, CMULines), and a third (CMUText) will almost always be needed. The additional feature classes CMUMapUnitLines and CMUMapUnitPoints may be needed as well (see discussion below).

\section{CMUMapUnitPolys (Polygon Feature Class)}

The CMUMapUnitPolys polygon feature class contains the units within the CorrelationOfMapUnits feature dataset. Table 2-1 describes the fields (and their values) in the CMUMapUnitPolys polygon feature class.

Outlines of CMU unit boxes may be drawn either by symbolizing the edges of CMUMapUnitPolys polygons or by symbolizing coincident lines recorded in feature class CMULines (see below). Ghost boxes, which are empty CMU boxes sometimes used to depict the protoliths of metamorphic-rock units, may be shown in the CMUMapUnitPolys polygon feature class by setting the Symbol value = "blank" or null. Alternatively, the empty CMU box outlines could be stored alone in the CMULines line feature class, as discussed below.

\section{CMULines (Line Feature Class)}

Any brackets, horizontal rules, or leaders (and sometimes, outlines of ghost boxes) in the CMU diagram are stored in the CMULines feature class. Outlines of CMU unit boxes may be stored in this feature class, or they may be the symbolized edges of CMUMapUnitPolys polygons. Table 2-2 describes the fields (and their values) in the CMULines line feature class.

\section{CMUMapUnitLines and CMUMapUnitPoints (Optional Line and Point Feature Classes)}

If map units have been depicted on the map graphic as line or point features because they are too small to show at the map scale, they will need to be depicted in the CMU that way as well. If such is the case, then feature classes CMUMapUnitLines and (or) CMUMapUnitPoints are needed. A CMUMapUnitLines feature class should have an_ID field named CMUMapUnitLines_ID, and the Symbol field should reference a line symbol. Other field names, values, and usage should be identical to those shown in table 2-1. Similarly, a CMUMapUnitPoints feature class should have an_ID field named CMUMapUnitPoints_ID, and the Symbol field should reference a point symbol. Other field names, values, and usage should be the identical to those shown in table 2-1. 
Table 2-1. Fields in CMUMapUnitPolys (a polygon feature class in the CorrelationOfMapUnits feature dataset).

[Abbreviation: CMU, Correlation of Map Units. See also, tables 7, 11, 12, 14]

\begin{tabular}{|c|c|c|}
\hline Field name & Description & Notes \\
\hline MapUnit & $\begin{array}{l}\text { Short, easily understood ASCII-character }{ }^{1} \text { identifier } \\
\text { for the map unit represented by this CMU box. } \\
\text { Foreign key to DescriptionOfMapUnits table }\end{array}$ & $\begin{array}{l}\text { Examples of values are "Qal", "Tg", "Kit", "Trdu" (see } \\
\text { table 11). Use of special characters is discouraged. Null } \\
\text { values not permitted }\end{array}$ \\
\hline Label & $\begin{array}{l}\text { Describes map-unit label for this CMU box. } \\
\text { Field from which map-unit label in CMU is } \\
\text { generated }\end{array}$ & $\begin{array}{l}\text { May or may not be same as MapUnit value. Allows for special } \\
\text { fonts to show geologic age symbols or other non-ASCII } \\
\text { characters (see table 11). Generally, is equal to value in } \\
\text { DescriptionOfMapUnits table. Null values permitted for } \\
\text { unlabeled CMU boxes (for example, ghost boxes) }\end{array}$ \\
\hline Symbol & References an area-fill symbol & $\begin{array}{l}\text { Area-fill symbols (map-unit color, pattern, or color+pattern) } \\
\text { must be included in accompanying .style file. Null values } \\
\text { permitted only for CMU boxes that are not filled with color } \\
\text { or pattern (for example, ghost boxes) }\end{array}$ \\
\hline CMUMapUnitPolys_ID & Primary key & $\begin{array}{c}\text { Examples of values are "CMUMUP1", "CMUMUP2". Values } \\
\text { must be unique in database. Null values not permitted }\end{array}$ \\
\hline
\end{tabular}

${ }^{1}$ ASCII, American Standard Code for Information Interchange; ASCII characters mainly consist of lower and upper case letters, numerals, and certain punctuation characters (specifically, ones that can be accessed using the Shift key).

Table 2-2. Fields in CMULines (a line feature class in the CorrelationOfMapUnits feature dataset).

[Abbreviation: CMU, Correlation of Map Units. Content of fields in magenta type (in this case, the Type field) must be defined in Glossary table]

\begin{tabular}{lcc}
\hline \multicolumn{1}{c}{ Field name } & \multicolumn{1}{c}{ Description } & Notes \\
\hline Type & $\begin{array}{c}\text { Specifies type of line feature represented by this } \\
\text { database row }\end{array}$ & $\begin{array}{c}\text { Examples of values are "CMU box border", "CMU } \\
\text { leader", "CMU rule", "CMU bracket". Values must be } \\
\text { defined in Glossary table. Null values not permitted }\end{array}$ \\
Symbol & References a line symbol & $\begin{array}{c}\text { Line symbols must be included in accompanying .style } \\
\text { file. Null values permitted }\end{array}$ \\
CMULines_ID & Primary key & $\begin{array}{c}\text { Examples of values are "CMULIN1", "CMULIN2". Values } \\
\text { must be unique in database. Null values not permitted }\end{array}$ \\
\hline
\end{tabular}

\section{CMUText (Annotation Feature Class)}

Annotation text in the CMU, as well as annotation attributes such as font, font size, font effects, and text angle, are stored in the CMUText annotation feature class. Esri's ArcGIS dictates the fields for this type of feature class; thus, they are not described here.

\section{MiscellaneousMapInformation (Nonspatial Table)}

Most published map reports have a significant amount of miscellaneous information printed in the collar area around the map (that is, the map margin). Such miscellaneous information may include the title, authorship, publication date, publishing agency, publication series and number, scale, geologic mapping credit, data-compilation credit, GIS database and cartography credit, editing credit, cartographic-production credit, manuscript approval date, local magnetic declination, base-map information, trade-name and other disclaimers, publication URL, International Standard Serial Number(s) (ISSNs) ${ }^{14}$ and suggested citation. This information is helpful for a full understanding of the associated database and could be captured in the database in the MiscellaneousMapInformation table.

A common characteristic of these map marginalia is that they are single statements that apply to the map as a whole; accordingly, information can be harvested from the MiscellaneousMapInformation table to populate formal metadata. However, the details of this information can vary from map to map and from agency to agency, and so we do not attempt to prescribe which map properties should be encoded in the table nor what they should be named. Table 2-3 describes the fields (and their values) in a MiscellaneousMapInformation table.

${ }^{14}$ International Standard Serial Numbers (ISSNs) for online and printed versions of published reports are assigned by the Library of Congress. 


\section{StandardLithology (Nonspatial Table)}

A mapmaker may choose to provide descriptions of map units that are more detailed than the GeoMaterial values and more structured than the free-text in the Description field in the DescriptionOfMapUnits table. StandardLithology (described in table 2-4) provides a structure for such descriptions of geologic map units.

Alternatively, a mapmaker may choose to create a table (form not specified here) for lithologic classification that best suits the geology of the map area and the intended audience. Such a table could differ significantly from StandardLithology.

The StandardLithology table represents the lithologic composition(s) of map units by associating the unit with one or more lithology categories from CGI's "s "Simple Lithology" controlled vocabulary (available at http://resource.geosciml.org/def/voc/; see discussion in the "GeoMaterial Terms" section, in appendix 1 above).

${ }^{15} \mathrm{CGI}$, Commission for the Management and Application of Geoscience Information.

Table 2-3. Fields in MiscellaneousMapInformation (a nonspatial table).

\begin{tabular}{|c|c|c|}
\hline Field name & Description & Notes \\
\hline MapProperty & $\begin{array}{l}\text { Name of map } \\
\text { property }\end{array}$ & $\begin{array}{l}\text { Examples of values are "scale", "authors and affiliations", "magnetic declination", } \\
\text { "date of approval". Null values not permitted }\end{array}$ \\
\hline MapPropertyValue & $\begin{array}{l}\text { Value of map } \\
\text { property }\end{array}$ & $\begin{array}{l}\text { Examples of values are "1:24,000", "G.S. Smith1 and J. Doe2, 1-Division of } \\
\text { Geology, Some State, 2-Big University", "16.5 degrees", "approved for } \\
\text { publication on } 23 \text { September } 2017 \text { ". Null values not permitted }\end{array}$ \\
\hline MiscellaneousMapInformation_ID & Primary key & $\begin{array}{l}\text { Examples of values are "MMI01", "MMI03". Values must be unique in database. } \\
\text { Null values not permitted }\end{array}$ \\
\hline
\end{tabular}

Table 2-4. Fields in StandardLithology (a nonspatial table).

[Abbreviation: CGI, Commission for the Management and Application of Geoscience Information. Content of fields in magenta type (in this case, the ScientificConfidence field) must be defined in Glossary table. See also, tables 11, 14, 2-5]

\begin{tabular}{|c|c|c|}
\hline Field name & Description & Notes \\
\hline MapUnit & $\begin{array}{l}\text { Short, easily understood ASCII-character }{ }^{1} \text { identifier } \\
\text { for the label for this map unit. Foreign key to } \\
\text { DescriptionOfMapUnits table }\end{array}$ & $\begin{array}{l}\text { Examples of values are "Qal", "Tg”, "Kit" (see table 11). } \\
\text { Null values not permitted }\end{array}$ \\
\hline ProportionTerm & $\begin{array}{l}\text { Indicates proportion (as qualitative term) of this map unit in } \\
\text { which lithology is found. Recommended domain is CGI's } \\
\text { Proportion Term vocabulary (available at http://resource. } \\
\text { geosciml.org/def/voc/); however, map producers may wish } \\
\text { to restrict vocabulary to a shorter and less expressive, but } \\
\text { easier to use, list (see discussion in text below) }\end{array}$ & $\begin{array}{l}\text { Examples of values are "dominant", "variable", "rare". } \\
\text { Null values permitted; however, value in either } \\
\text { ProportionValue or ProportionTerm should be non-null }\end{array}$ \\
\hline ScientificConfidence & $\begin{array}{l}\text { Indicates how confidently existence and identity of lithology } \\
\text { is identified as being found within this map unit }\end{array}$ & $\begin{array}{l}\text { Examples of values are "existence certain", "identity } \\
\text { uncertain". Values must be defined in Glossary table. } \\
\text { Null values not permitted; suggest setting default value } \\
\text { to "existence and identity certain" }\end{array}$ \\
\hline DataSourceID & $\begin{array}{l}\text { Identifies source of StandardLithology description. } \\
\text { Foreign key to DataSources table }\end{array}$ & Null values not permitted \\
\hline StandardLithology_ID & Primary key & $\begin{array}{l}\text { Example values are "STL1", "STL2". Values must be } \\
\text { unique in database. Null values not permitted }\end{array}$ \\
\hline
\end{tabular}

${ }^{1}$ ASCII, American Standard Code for Information Interchange; ASCII characters mainly consist of lower and upper case letters, numerals, and certain punctuation characters (specifically, ones that can be accessed using the Shift key). 
Note that descriptions for a single map unit may span several rows in this table. This allows the description of multipart (for example, interbedded) units using a quantitative or qualitative description of the relative abundance of each component. Each associated lithology category has a PartType field that indicates how the rock type is found within the unit (for example, vein or dike lithosome, layer lithosome, stratigraphic part, inclusion, blocks, etc.) and either a ProportionTerm field (a qualitative term) or a ProportionValue field (a numeric value) that indicates its proportion within the unit.

For values in the ProportionTerm field, CGI's Proportion Term list (available at http://resource.geosciml.org/def/voc/) is recommended. But for parsing certain map-unit descriptions, especially those for already compiled and published maps, into a controlled-term list, a simpler list of proportion terms whose definitions are less precise may be more appropriate, particularly the case in which the percentage proportions, especially among the dominant lithologic constituents, cannot readily be determined. Such a list might include the following proportion terms (highlighted in bold type):

- all - the lithology constitutes all of the map unit

- major - the lithology is a major or significant component of the map unit

- minor - the lithology is a minor or relatively insignificant component of the map unit

- trace - the lithology is present but is a very small component of the map unit

Table 2-5 lists some examples of StandardLithology records. Use either the ProportionTerm field or the ProportionValue field as appropriate; note that, for a given record, only one value is permitted to be null. The ProportionValue values are fractional, ranging between 0.0 and 1.0 ; for a single map unit, these values should sum to no more than 1.0.

If you generate records in a StandardLithology table by interpreting map-unit descriptions in an existing map or database, we recommend that you set the DataSourceID value to point to an entry in the DataSources table, such as "DAS2", Source = "Smith, J.G., 1899, Geologic map of XYZ quadrangle: USGS GQ 9999, scale 1:125,000. Georeferenced and digitized by authors of this report", or something similar (see table 6).

Table 2-5. Examples of records in a StandardLithology table.

[Each row represents separate data instance. Values in PartType, Lithology, and ProportionTerm fields are from CGI's (Commission for the Management and Application of Geoscience Information's) Geologic Unit Part Role, Simple Lithology, and Proportion Term vocabularies, respectively (available at http:// resource.geosciml.org/def/voc/). See also, table 2-4]

\begin{tabular}{lclll}
\hline \multicolumn{1}{c}{ StandardLithology_ID } & MapUnit & \multicolumn{1}{c}{ PartType } & \multicolumn{1}{c}{ Lithology } & \multicolumn{1}{c}{ ProportionTerm } \\
\hline STL026 & $\mathrm{Tx}$ & Bed lithosome & Generic sandstone & Dominant \\
STL327 & $\mathrm{Tx}$ & Stratigraphic part & Siltstone & Minor \\
STL579 & $\mathrm{Tx}$ & Stratigraphic part & Chalk & Minor \\
STL264 & $\mathrm{Txt}$ & Bed lithosome & Siltstone & Dominant \\
STL265 & $\mathrm{Kit}$ & Only part & Tonalite & Dominant \\
STL266 & $\mathrm{KJz}$ & Bed lithosome & Limestone & Dominant \\
STL770 & $\mathrm{KJz}$ & Bed lithosome & Generic mudstone & Subordinate \\
\hline
\end{tabular}

\section{Deprecated Nonspatial Tables}

The NCGMP09 v.1.1 (U.S. Geological Survey National Cooperative Geologic Mapping Program, 2010) specification described the optional nonspatial tables ExtendedAttributes and GeologicEvents, which could be used to specify arbitrary properties and their values for any feature in the map database, as well as to attach multiple ages to map units or map features. To our knowledge, these tables were rarely, if ever, implemented; therefore, they have been omitted from this GeMS specification. If map authors or publishers wish to encode multiple ages or to link otherwise unspecified attributes to some items within the database, they may consult the NCGMP09 v.1.1 documentation and specification.

\section{Reference Cited in Appendix 2}

U.S. Geological Survey National Cooperative Geologic Mapping Program, 2010, NCGMP09—Draft standard format for digital publication of geologic maps, version 1.1, in Soller, D.R., ed., Digital Mapping Techniques '09—Workshop Proceedings: U.S. Geological Survey Open-File Report 2010-1335, p. 93-146, 4 appendixes, https://pubs.usgs.gov/of/2010/1335/pdf/usgs_of20101335_NCGMP09.pdf. 


\section{Appendix 3. Parsing a Description of Map Units (DMU) into the DescriptionOfMapUnits Table and the HierarchyKey Field}

\section{The DescriptionOfMapUnits Table}

Parsing a Description of Map Units (DMU) into the fields of the DescriptionOfMapUnits table is a relatively straightforward process. As an example, figure 3-1 shows a heading and two map unit descriptions from a DMU in a relatively recent published geologic map (Evarts and others, 2016); table 3-1 shows the same heading and two map units parsed into the fields of a DescriptionOfMapUnits table.

Note that several fields are missing from table 3-1. The person who translates an existing map into the GeMS schema will have to determine appropriate values of, for example, the FullName, GeoMaterial, Symbol, Pattern, and AreaFillRGB fields. The following list contains a few things to keep in mind when translating a map into GeMS:

- Values in the MapUnit and Label fields commonly, but not always, are identical (see table 11)

- The Label field allows for the use of special characters such as ${ }^{*}$, , and $\}$, which translate into the geologic age symbols $\mathbf{k}, \mathbb{P}$, and $\mathrm{Mz}$, respectively, when the FGDCGeoAge font is specified (see table 11)

- Unused fields (for example, the MapUnit and Label fields for headings) are filled with null values, not empty strings

- It is not necessary to add an entry for the DMU title

- Formatting such as bolding of unit names and ages and parentheses around the unit ages should not be captured in the DescriptionOfMapUnits table, as it can be predicted from the table structure. Other kinds of formatting (for example, superscripts or paragraph breaks) cannot be easily stored in the Name and Description fields; if such formatting is important to preserve, use HTML-style markup

Note that some older geologic maps and maps published by some agencies may not have DMUs that are formatted as is shown in figure 3-1 (see the examples given in "The HierarchyKey Field" section below). Nevertheless, we have not found a DMU or map-unit explanation that cannot be translated into this schema.

\section{The HierarchyKey Field}

Map-unit explanations on geologic maps commonly are ordered and hierarchical: map units are listed in an intentional sequence; they usually are listed under headings and subheadings; and some may be subunits of other map units. The sequence of map units shown in a DMU generally corresponds to the relative ages of map units, from youngest to oldest. This same hierarchy also is commonly expressed in a Correlation of Map Units (CMU) diagram. Hierarchy also may express closeness in genesis, paleogeography, relative certainty of map-unit identification, or other relations. On some maps that organize the DMU by the various physiographic regions or geologic terranes, or where many units were deposited contemporaneously (for example, many surficial geologic maps), the rules for designation of hierarchy may appear somewhat arbitrary, but the hierarchy is evident from the ordering and indentation of the DMU.

In the DMU of a USGS geologic map, hierarchy is shown by the paragraph style (font style, alignment, and indentation) of successive elements. This hierarchy is also shown by the spatial arrangement of map-unit boxes, headings, and brackets in the associated CMU diagram.

We record these relations in the DescriptionOfMapUnits table with the HierarchyKey attribute, which (1) allows the table to be sorted in its proper sequence, and (2) records parent-child relations. Values of HierarchyKey are text strings with the form of $n n-n n$ or $n n-n n-n n$, etc. Each fragment ( $n n)$ of the HierarchyKey value is numeric, is left-padded with zeros so that each fragment has the same length, and is dash delimited. Different values of HierarchyKey may be made up of different numbers of fragments. For example, a DMU row that has a HierarchyKey value $=$ "03-11" is the eleventh child of the parent row that has the HierarchyKey value $=$ "03".

The following are some general rules for the construction of HierarchyKey values:

- Within siblings (that is, children of the same parent), elements are numbered youngest to oldest

- A parent may have 0,1 , or many children

- The length ( $n$ versus $n n$ versus $n n n)$ of a HierarchyKey fragment is determined by the size of the family that has the largest number of siblings. Note that it is acceptable to have extra zeros (for example, using an unnecessarily long fragment value of " 003 ", when "3" or "03" would work), but this practice generally is discouraged as it reduces the readability of HierarchyKey values 


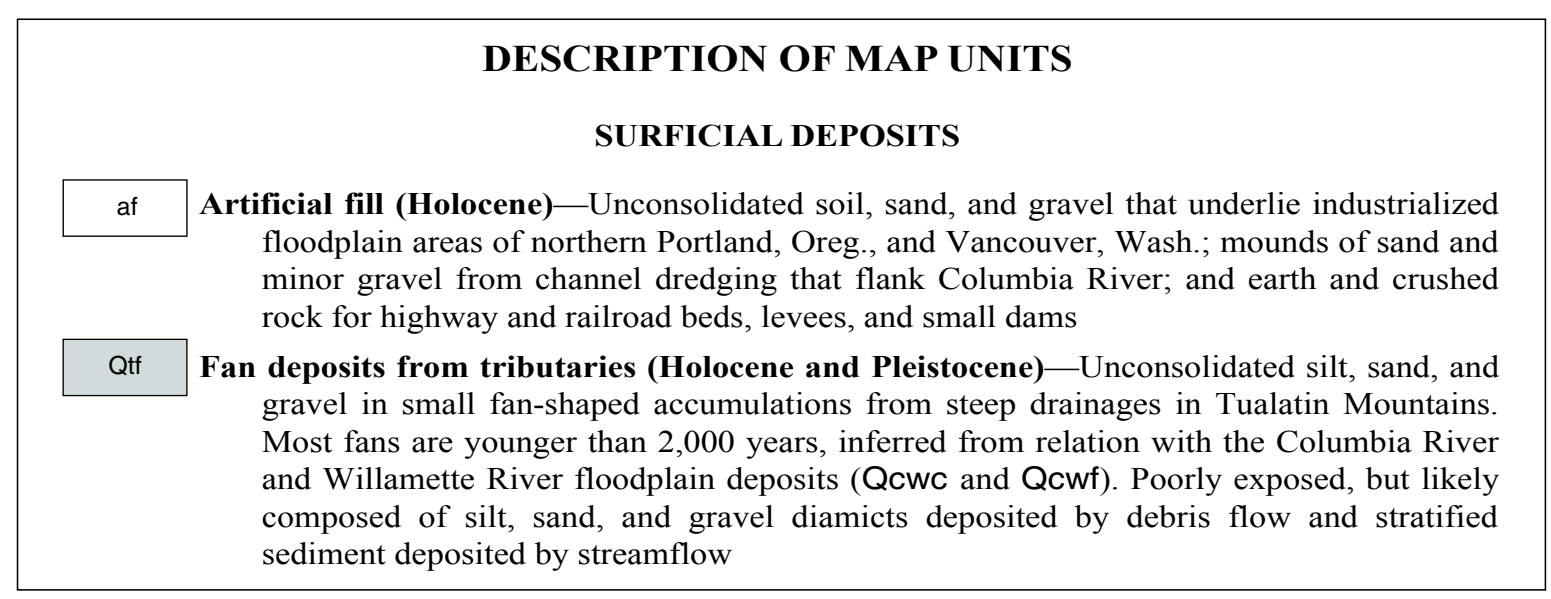

Figure 3-1. Part of a modern Description of Map Units, excerpted from a geologic map published relatively recently (Evarts and others, 2016). See table 3-1 for parsing of heading and map units into a DescriptionOfMapUnits table.

Table 3-1. Selected fields and values from a DescriptionOfMapUnits table for the Description of Map Units depicted in figure 3-1.

[Heading, map units, unit names, ages, and descriptions from Evarts and others (2016). Note that title ("DESCRIPTION OF MAP UNITS"; ParagraphStyle "DMUHeading1") is omitted]

\begin{tabular}{|c|c|c|c|c|c|}
\hline \multicolumn{6}{|c|}{ Field name } \\
\hline MapUnit & Label & Name & Age & Description & ParagraphStyle \\
\hline$<$ null $>$ & $<$ null $>$ & Surficial deposits & $<$ null $>$ & $<$ null $>$ & DMUHeading2 \\
\hline af & af & Artificial fill & Holocene & $\begin{array}{l}\text { Unconsolidated soil, sand, and gravel that underlie } \\
\text { industrialized floodplain areas of northern Portland, } \\
\text { Oreg., and Vancouver, Wash.; mounds of sand and } \\
\text { minor gravel from channel dredging that flank } \\
\text { Columbia River; and earth and crushed rock for } \\
\text { highway and railroad beds, levees, and small dams }\end{array}$ & DMUUnit1 \\
\hline Qtf & Qtf & $\begin{array}{l}\text { Fan deposits from } \\
\text { tributaries }\end{array}$ & $\begin{array}{l}\text { Holocene and } \\
\text { Pleistocene }\end{array}$ & $\begin{array}{l}\text { Unconsolidated silt, sand, and gravel in small fan-shaped } \\
\text { accumulations from steep drainages in Tualatin } \\
\text { Mountains. Most fans are younger than 2,000 years, } \\
\text { inferred from relation with Columbia River and } \\
\text { Willamette River floodplain deposits (Qcwc and } \\
\text { Qcwf). Poorly exposed, but likely composed of silt, } \\
\text { sand, and gravel diamicts deposited by debris flow and } \\
\text { stratified sediment deposited by streamflow }\end{array}$ & DMUUnit1 \\
\hline
\end{tabular}

- Headings cannot be children of map units; however, headings may be children of other headings, and map units may be children of either headings or other map units

- Values of HierarchyKey within a single DescriptionOfMapUnits table must be unique

- If an alphanumeric sort on the HierarchyKey field (in ascending order) does not put the DescriptionOfMapUnits table in the correct order (that is, if it does not match the order of the DMU), then one or more HierarchyKey values are incorrect

- After alphanumeric sorting, a HierarchyKey value may be the same length as its preceding value (that is, it is a sibling); or it may be one fragment longer than its preceding element (that is, it is a child) but not more than one fragment longer (generations cannot be omitted); or it may be shorter than its preceding value (that is, it is an aunt, a great-aunt, etc.; in other words, it is not in the immediate family)

- It typically is easier to decipher hierarchy from a DMU than from a CMU. Unfortunately, on some published maps, the hierarchy of headings and map units in the DMU and CMU do not agree, and it is not easy to determine which is correct. When encoding such maps, choose the hierarchy that you think best expresses the author's intentions and will best serve users of the database.

The figures and tables below are examples that illustrate the use of the HierarchyKey field and its values. Each figure (figs. 3-2 through 3-5) reproduces part of a DMU or CMU for a published map, and each table (tables 3-2 through 3-5) reproduces part of the corresponding DescriptionOfMapUnits table. 


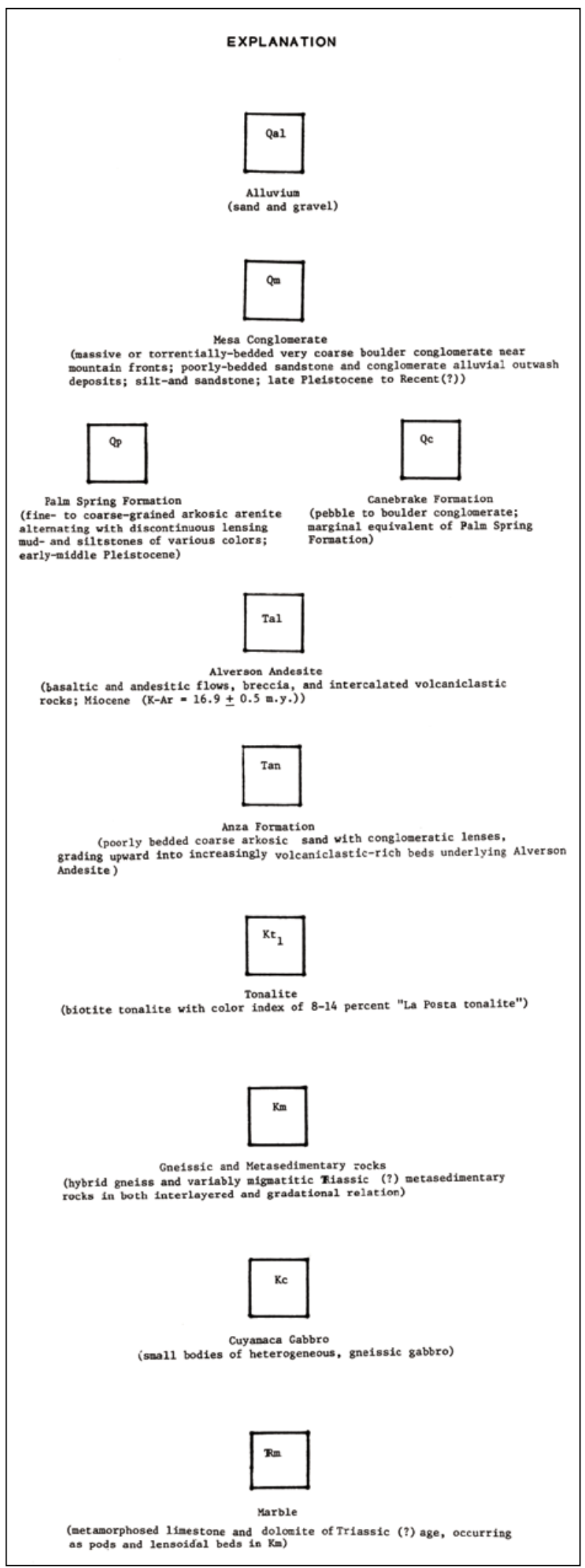

Figure 3-2. Reproduction of part of a map-unit explanation from a somewhat older geologic map (Hoggatt, 1979; image extracted from map accessed from https://ngmdb.usgs.gov/mapview//, showing an older style of map-unit description. See table 3-2 for parsing of map units into a DescriptionOfMapUnits table.
Table 3-2. Selected fields and values from a DescriptionOfMapUnits table for the explanation depicted in figure 3-2.

[Map units and unit names from Hoggatt (1979). Note that title ("EXPLANATION") is omitted]

\begin{tabular}{cccl}
\hline \multicolumn{4}{c}{ Field name } \\
\hline HierarchyKey & ParagraphStyle & MapUnit & \multicolumn{1}{c}{ Name } \\
\hline 01 & DMUUnit1 & Qal & Alluvium \\
02 & DMUUnit1 & Qm & Mesa Conglomerate \\
03 & DMUUnit1 & Qp & Palm Spring Formation \\
04 & DMUUnit1 & Qc & Canebrake Formation \\
05 & DMUUnit1 & Tal & Alverson Andesite \\
06 & DMUUnit1 & Tan & Anza Formation \\
07 & DMUUnit1 & Kt1 & Tonalite \\
08 & DMUUnit1 & Km & Gneissic and \\
& & & $\begin{array}{l}\text { Metasedimentary } \\
\text { rocks }\end{array}$ \\
09 & DMUUnit1 & Kc & Cuyamaca Gabbro \\
10 & DMUUnit1 & Trm & Marble \\
\hline
\end{tabular}

Note the following in the above examples (fig. 3-2; table 3-2):

- There is no separate DMU in this report; map-unit descriptions are contained in the Explanation

- The Explanation (fig. 3-2) has no headings or subunits

- The sequence of map units (top to bottom, left to right) is in the order of increasing geologic age (the Palm Spring Formation and the Canebrake Formation have the same age), and every map unit has the same rank. Thus, the HierarchyKey field contains sequential values of 1 to 10, left-padded with zeroes so that an alphanumeric sort on HierarchyKey puts the rows of the DescriptionOfMapUnits table in the correct sequence

- The last entry (Name = "Marble", HierarchyKey = "10") has a MapUnit value = "Trm", which is an ASCIIcharacter substitution for the map-unit label $\mathrm{km}$. To produce the geologic age symbol $\mathrm{k}$ ( = Triassic) on the map, the Label (field not shown) value would be “^”, and the FGDCGeoAge font would need to be specified (see table 11); thus, for MapUnit = "Trm", Label = "^ $\mathrm{m}$ ".

Note that, in a database, values in the Name field consist of unformatted ASCII characters. ${ }^{16}$ However, for purposes of illustration only, the contents of the Name field in the tables that follow (tables 3-3 through 3-5) have been formatted (by alignment, font style, and capitalization) so it will be easier to match them to their corresponding entries in the DMU or CMU on the source map. In an actual database, such formatting would only be recorded in the ParagraphStyle field.

\footnotetext{
${ }^{16}$ ASCII, American Standard Code for Information Interchange; ASCII characters mainly consist of lower and upper case letters, numerals, and certain punctuation characters (specifically, ones that can be accessed using Shift key).
} 
Figure 3-3. Part of a modern Correlation of Map Units diagram, excerpted from a geologic map published relatively recently (Evarts and others, 2016). See table 3-3 for parsing of headings and map units into a DescriptionOfMapUnits table.

\section{BASIN-FILL DEPOSITS}
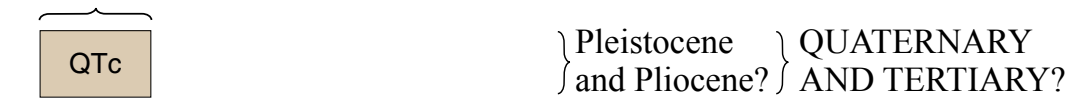

Unconformity

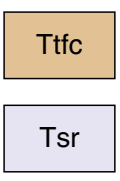

$$
\left\{\begin{array}{l}
\text { Pliocene } \\
\text { and (or) } \\
\text { Miocene }
\end{array}\right.
$$

Unconformity

BEDROCK

\section{COLUMBIA RIVER BASALT GROUP}

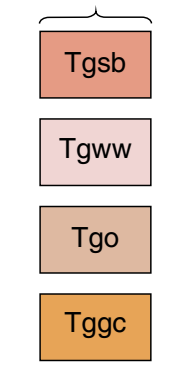

Unconformity

PALEOGENE SEDIMENTARY ROCKS

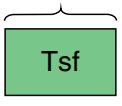

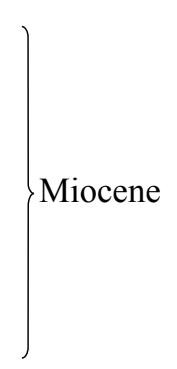

TERTIARY

Oligocene

Table 3-3. Selected fields and values from a DescriptionOfMapUnits table for the Correlation of Map Units diagram depicted in figure 3-3.

\begin{tabular}{|c|c|c|c|}
\hline \multicolumn{4}{|r|}{ Field name } \\
\hline HierarchyKey & ParagraphStyle & MapUnit & Name \\
\hline 2 & DMUHeading2 & $<$ null $>$ & BASIN-FILL DEPOSITS \\
\hline $2-1$ & DMUUnit1 & QTc & Unnamed conglomerate \\
\hline $2-2$ & DMUUnit1 & Ttfe & Troutdale Formation, conglomerate member \\
\hline $2-3$ & DMUUnit1 & Tsr & Sandy River Mudstone \\
\hline 3 & DMUHeading2 & $<$ null $>$ & BEDROCK \\
\hline $3-1-1-1$ & DMUUnit2 & Tgsb & Sentinel Bluffs Member \\
\hline $3-1-1-2$ & DMUUnit2 & Tgww & Winter Water Member \\
\hline $3-1-1-3$ & DMUUnit2 & Tgo & Ortley member \\
\hline $3-1-1-4$ & DMUUnit2 & Tgge & Grouse Creek member \\
\hline $3-2$ & DMUHeading3 & $<$ null $>$ & PALEOGENE SEDIMENTARY ROCKS \\
\hline
\end{tabular}

[Headings and map units from Evarts and others (2016); unit names from Description of Map Units (not shown). Note that formatting (font style, capitalization, alignment, and indentation) of Name values is shown for example purposes only, to help match them to headings and units shown in figure 3-3] 


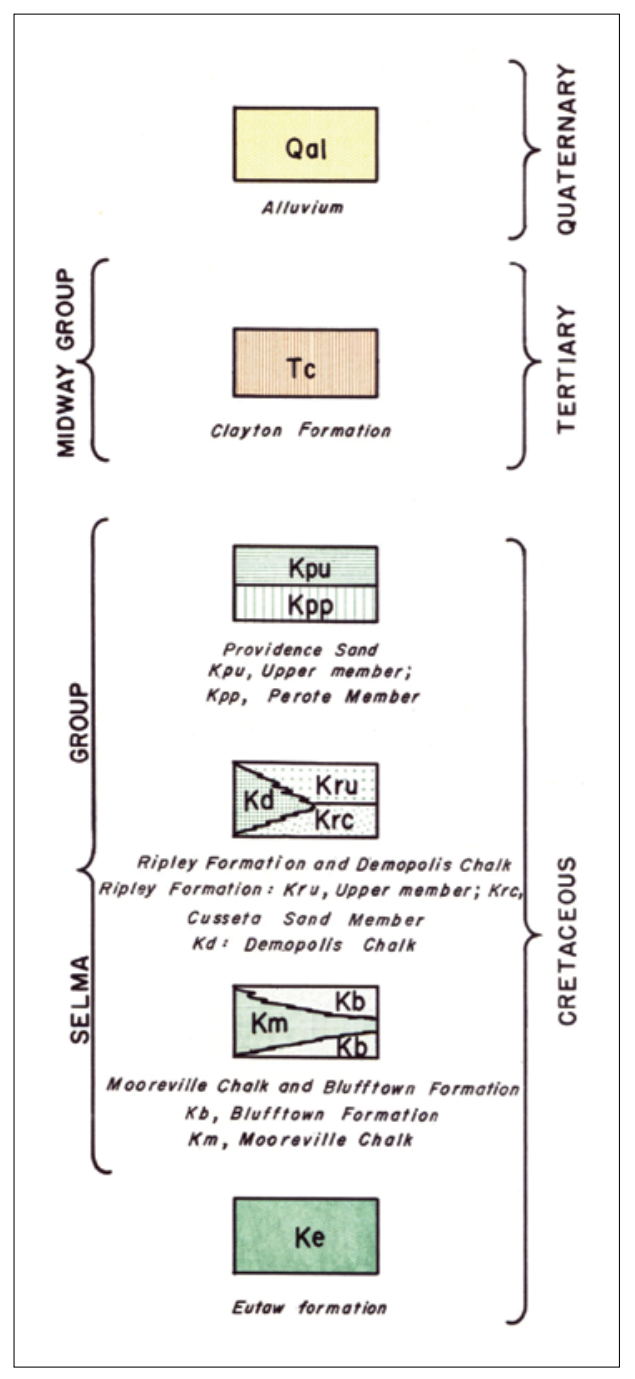

Note the following in the above examples (fig. 3-3; table 3-3):

- The CMU title (not shown in figure 3-3) was not considered when other HierarchyKey values in the DescriptionOfMapUnits table (table 3-3) were assigned

- Also not shown in figure 3-3 are younger surficial deposits (listed under the heading "SURFICIAL DEPOSITS"); however, these units and their heading were considered when assigning HierarchyKey values for the subsequent headings and units (HierarchyKey $=$ "2", "2-1", etc.)

- The "unconformity" notations in the CMU were not entered into the DescriptionOfMapUnits table

- Unused fields (for example, the MapUnit field for headings) are filled with null values, not empty strings

- The authors of this map chose to treat the Columbia River Basalt Group, which is a formal lithostratigraphic unit, as a heading, not a map unit. The Grande Ronde Basalt, which also is a formal lithostratigraphic unit, also is not mapped separately; however, the authors chose to treat it as a map unit. The ParagraphStyle attribute is needed to distinguish these choices (headings versus map units)

Figure 3-4. Part of an older map-unit explanation, excerpted from an older geologic map (Scott, 1961), showing an older, abbreviated style of mapunit description. See table 3-4 for parsing of headings and map units into DescriptionOfMapUnits table.

Table 3-4. Selected fields and values from a DescriptionOfMapUnits table for the explanation depicted in figure 3-4.

[Headings, map units, and unit names from Scott (1961). Note that formatting (capitalization, alignment, and perceived indentation) of Name values is shown for example purposes only, to help match them to headings and units shown in figure 3-4. Note also that title ("EXPLANATION") is omitted]

\begin{tabular}{|c|c|c|c|}
\hline \multicolumn{4}{|c|}{ Field name } \\
\hline HierarchyKey & ParagraphStyle & MapUnit & Name \\
\hline 1 & DMUUnit1 & Qal & Alluvium \\
\hline 2 & DMUHeading2 & $<$ null $>$ & MIDWAY GROUP \\
\hline $2-1$ & DMUUnit1 & $\mathrm{Tc}$ & Clayton Formation \\
\hline 3 & DMUHeading2 & $<$ null $>$ & SELMA GROUP \\
\hline $3-1$ & DMUUnit1 & $<$ null $>$ & Providence Sand \\
\hline $3-1-1$ & DMUUnit2 & $\mathrm{Kpu}$ & Upper member \\
\hline $3-1-2$ & DMUUnit2 & Kpp & Perote Member \\
\hline $3-2$ & DMUUnit1 & $<$ null $>$ & Ripley Formation and Demopolis Chalk \\
\hline $3-2-1$ & DMUUnit2 & $\mathrm{Kd}$ & Demopolis Chalk \\
\hline $3-2-2$ & DMUUnit2 & $<$ null $>$ & Ripley Formation \\
\hline $3-2-2-1$ & DMUUnit3 & Kru & Upper member \\
\hline $3-2-2-2$ & DMUUnit3 & $\mathrm{Krc}$ & Cusseta Sand Member \\
\hline $3-3$ & DMUUnit1 & $<$ null $>$ & Mooreville Chalk and Blufftown Formation \\
\hline $3-3-1$ & DMUUnit2 & $\mathrm{Km}$ & Mooreville Chalk \\
\hline $3-3-2$ & DMUUnit2 & $\mathrm{Kb}$ & Blufftown Formation \\
\hline 4 & DMUUnit1 & $\mathrm{Ke}$ & Eutaw formation \\
\hline
\end{tabular}


Note the following in the above examples (fig. 3-4; table 3-4):

- There is no separate DMU in this report; map-unit descriptions are contained in the Explanation

- Unused fields (for example, the MapUnit field for headings) are filled with null values, not empty strings

- It is unclear whether Midway Group and Selma Group are headings or map units, but the hierarchy expressed in the Explanation is obvious, and the corresponding HierarchyKey values ("2", "3") are easily assigned. In this case, the ParagraphStyle values ("DMUHeading2") were chosen somewhat arbitrarily

- The Explanation boxes (as read from left to right, top to bottom) show the Demopolis Chalk preceding the Ripley Formation, but the order of the associated text suggests the reverse. In this case, the HierarchyKey values ("3-2-1", "3-2-2") chosen reflect the box order.

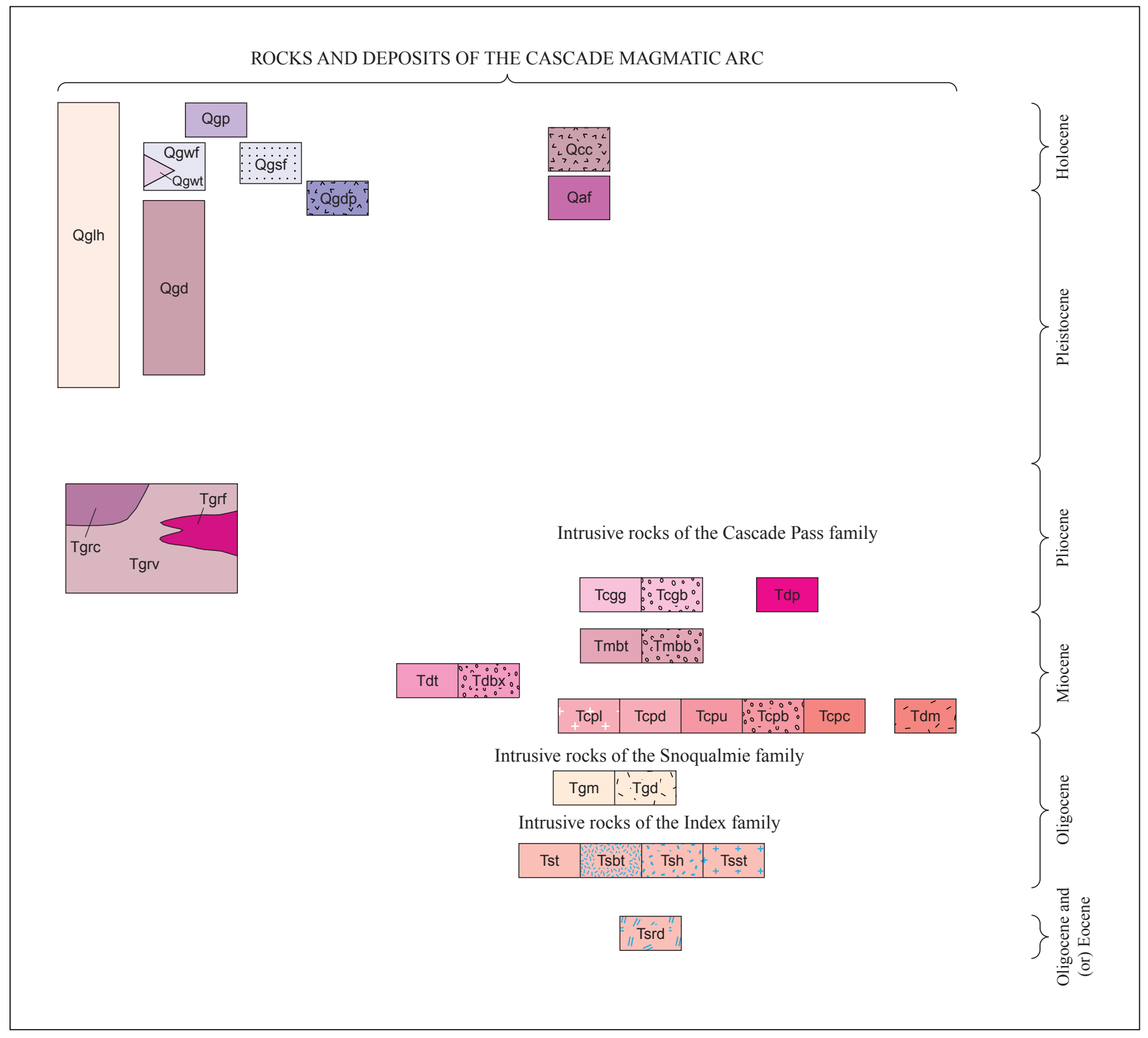

Figure 3-5. Part of a relatively modern Correlation of Map Units diagram, excerpted from a geologic map published somewhat recently (Tabor and others, 2002). See table 3-5 for parsing of headings and map units into DescriptionOfMapUnits table. 
Table 3-5. Selected fields and values from a Description OfMapUnits table for the Correlation of Map Units diagram depicted in figure 3-5.

[Headings and map units from Tabor and others (2002); unit names from Description of Map Units (not shown). Note that formatting (font style, capitalization, alignment, and indentation) of Name values is shown for example purposes only, to help match them to headings and units shown in figure 3-5]

\begin{tabular}{|c|c|c|c|}
\hline \multicolumn{4}{|r|}{ Field name } \\
\hline HierarchyKey & ParagraphStyle & MapUnit & Name \\
\hline 4 & DMUHeading3 & $<$ null $>$ & ROCKS AND DEPOSITS OF THE CASCADE MAGMATIC ARC \\
\hline $4-1$ & DMUUnit1 & $<$ null $>$ & Rocks of Glacier Peak volcano and associated volcanic rocks and deposits \\
\hline $4-1-2$ & DMUUnit2 & Qgp & Pumice deposits \\
\hline $4-1-3$ & DMUUnit2 & Qgwf & Deposits of the White Chuck fill \\
\hline $4-1-5$ & DMUUnit2 & Qgd & Dacite \\
\hline $4-1-6$ & DMUUnit2 & Qgdp & Dacite of Disappointment Peak \\
\hline $4-1-7$ & DMUUnit2 & Qcc & Cinder cones \\
\hline $4-1-8$ & DMUUnit2 & Qaf & Andesite flow \\
\hline $4-1-9$ & DMUUnit2 & $<$ null $>$ & Volcanic rocks of Gamma Ridge \\
\hline $4-2$ & DMUHeading4 & $<$ null $>$ & Intrusive rocks of the Cascade Pass family \\
\hline $4-2-1$ & DMUUnit1 & $<$ null $>$ & Cool Glacier stock \\
\hline $4-2-1-1$ & DMUUnit2 & Tcgg & Granodiorite \\
\hline $4-2-1-2$ & DMUUnit2 & Tcgb & Breccia \\
\hline $4-2-2$ & DMUUnit1 & Tdp & Dacite plugs and dikes \\
\hline $4-2-3$ & DMUUnit1 & $<$ null $>$ & Mount Buckindy pluton \\
\hline $4-2-3-1$ & DMUUnit2 & Tmbt & Tonalite and granodiorite \\
\hline $4-2-3-2$ & DMUUnit2 & Tmbb & Breccia \\
\hline $4-2-4$ & DMUUnit1 & $<$ null $>$ & Cascade Pass dike \\
\hline $4-2-4-1$ & DMUUnit2 & Tdt & Tonalite \\
\hline $4-2-6$ & DMUUnit1 & $\mathrm{Tdm}$ & Downey Mountain stock \\
\hline $4-3$ & DMUHeading4 & $<$ null $>$ & Intrusive rocks of the Snoqualmie family \\
\hline $4-3-1$ & DMUUnit1 & $<$ null $>$ & Grotto batholith \\
\hline $4-3-1-1$ & DMUUnit2 & Tgm & Monte Cristo stock \\
\hline $4-3-1-2$ & DMUUnit2 & Tgd & Dead Duck pluton \\
\hline $4-4$ & DMUHeading4 & $<$ null $>$ & Intrusive rocks of the Index family \\
\hline $4-4-1$ & DMUUnit1 & $<$ null $>$ & Squire Creek stock and related intrusive rocks \\
\hline $4-4-1-1$ & DMUUnit2 & Tst & Tonalite \\
\hline $4-4-1-2$ & DMUUnit2 & Tsbt & Biotite tonalite \\
\hline $4-4-1-3$ & DMUUnit2 & Tsh & Hornblende quartz diorite \\
\hline $4-4-1-4$ & DMUUnit2 & Tsst & Tonalite of the Shake Creek stock \\
\hline $4-4-2$ & DMUUnit1 & Tsrd & Sauk ring dike \\
\hline
\end{tabular}


Note the following in the above examples (fig. 3-5; table 3-5):

- The CMU title (not shown in figure 3-5) was not considered when other HierarchyKey values in the DescriptionOfMapUnits table (table 3-5) were assigned

- Also not shown in figure 3-5 are younger glacial and nonglacial deposits; however, these headings and units were considered when assigning HierarchyKey values for the subsequent headings and units

- The asterisks in some of the CMU boxes refer to the CMU bracketed headnote (not shown)

\section{References Cited in Appendix 3}

Evarts, R.C., O'Connor, J.E., and Cannon, C.M., 2016, Geologic map of the Sauvie Island quadrangle, Multnomah and Columbia Counties, Oregon, and Clark County, Washington: U.S. Geological Survey Scientific Investigations Map 3349, scale 1:24,000, pamphlet 34 p., https://doi.org/10.3133/sim3349.

Hoggatt, W.C., 1979, Geologic map of Sweeney Pass quadrangle, San Diego County, California: U.S. Geological Survey Open-File Report 79-754, scale 1:24,000, 36 p.

Scott, J.C., 1961, Geologic map of Bullock County, Alabama: Geological Survey of Alabama Special Map 19.

Tabor, R.W., Booth, D.B., Vance, J.A., and Ford, A.B., 2002, Geologic map of the Sauk River 30- by 60-minute quadrangle, Washington: U.S. Geological Survey Geologic Investigations Series Map I-2592, scale 1:100,000, 67 p., https://pubs.usgs.gov/imap/i2592/. 


\section{Appendix 4. Frequently Asked Questions (FA0s)}

How do I get started?

Scripts to create an empty GeMS database, to validate the GIS files and contents of the GeMS tables, and to export shapefile versions of the GeMS database are available at https://ngmdb.usgs.gov/Info/standards/GeMS/. The GeMS website also includes general guidance and examples of geologic maps published in the GeMS schema. Additional software tools and guidance are available at the GeMS Toolkit site on GitHub (https://github.com/usgs/GeMS_Tools).

I am publishing a new visualization of previously published digital geologic map data. Does this standard apply?

It could. However, GeMS was designed to apply to new geologic maps and to digital transcriptions (automations) of geologic maps that have previously been published in analog (paper or PDF) form. It has less applicability to new visualizations of previously published maps.

\section{How should I encode structure contours?}

You have at least two choices:

1. Structure contours may be encoded in an IsoValueLines feature class (for example, Type = "top $<$ Formation $X>$ ", or whatever is being contoured), along with a corresponding entry in the Glossary table for "top $<$ Formation $X>$ " that clearly defines the contoured surface. The Glossary table entry should also define the units used for the Value field (for example, meters above NAVD88) and the ValueConfidence field (for example, meters).

2. Alternately, you could create a new, appropriately named line feature class (for example, StructureContourLines) and an elevation attribute.

Generally speaking, we have found that contours are difficult to analyze automatically. The information contained in structure contours might be better stored in a raster (Esri grid) or triangulated irregular network (TIN) format.

How do I represent dikes?

Dikes are intrusive bodies of rock that have a finite extent. Again, you have several choices:

1. If the map is at a large enough scale and the mapping is detailed enough, dikes may be represented as (a) polygons (in the MapUnitPolys feature class) of a particular map unit (MapUnit = "Tvd"), as defined in the DescriptionOfMapUnits table, and (b) contacts (in the ContactsAndFaults feature class) that bound the polygons. This representation works well for wide dikes and large map scales; as the scale decreases and the dikes narrow, this representation does not work well.

2. Dikes that are (effectively, usually at smaller scales) too narrow to show as a polygon at the scale of the map graphic but are part of a map unit described in the DMU may be represented in a MapUnitLines feature class.

3. Dikes that are (effectively, usually at smaller scales) too narrow to show as a polygon at the scale of the map graphic and are not part of a map unit described in the DMU may be represented as line features (Type = "dike" or, perhaps, Type = "Tertiary andesite dike") in the GeologicLines feature class. Alternatively, they could be included in a DikesAndSills feature class.

How do I represent other small bodies of rock?

Small areas of a distinct rock type (for example, intrusive necks, limestone blocks in a continental-slope olistostrome, blueschist knockers in mélange) present similar choices as dikes, depending on scale:

1. You could represent them as small polygons in the MapUnitPolys feature class, bounded by contacts and (or) faults in the ContactsAndFaults feature class.

2. You could represent them as points in the MapUnitPoints feature class.

3. You could represent them as points (for example, Type = "intrusive neck" or "limestone block") in a GeologicPoints feature class.

What about bedrock contacts under alluvium?

There are several options, all of which are valid in GeMS:

1. Show concealed (dotted) bedrock contacts within alluvium (MapUnit = "Qal"; Name = "alluvium"; fill color, yellow). No feature in the database directly records the nature of the underlying bedrock; the map user does the implicit analysis, extrapolating from the adjoining mapped bedrock units, to figure out which bedrock units underlie the alluvium. This is the simplest, most commonly implemented option. 
2. If the map is a bedrock map, show the bedrock polygons and color them appropriately. Show the contacts between bedrock units as unconcealed (solid or dashed) contacts. Use an overlay polygon (perhaps symbolized with yellow dots or yellow diagonal lines) to show the extent of the overlying alluvium. If alluvium is described as a map unit in the Description of Map Units (DMU), the overlay is a polygon in the MapUnitOverlayPolys feature class (MapUnit = "Qal"), and "Qal" is defined in the DescriptionOfMapUnits table. If alluvium is described as a symbol in the Explanation of Map Symbols, the overlay is a polygon in the OverlayPolys feature class (Type = "alluvium"), and the term "alluvium" is defined in the Glossary table.

3. If the map is not a bedrock map, but one of the important bedrock units (important because it hosts economic silver mineralization) is covered by alluvium. You want the map user to immediately see where that bedrock unit is present, even where it is covered by alluvium. Show the buried bedrock contacts as concealed (dotted) lines and the alluvium as a yellow-filled polygon, as in option 1 (MapUnit = "Qal"; Name = "alluvium"); where the bedrock unit is present beneath alluvium, show a pattern (perhaps, fat diagonal dashed pink lines). You may think of this pattern as an underlay because it shows the map unit that underlies the alluvium, but cartographically (and in the database) it is an overlay pattern. Thus, the patterned area needs to be recorded as a polygon in one of the two ways that are discussed in option 2, either in the MapUnitOverlayPolys feature class (and defined in the DescriptionOfMapUnits table) or in the OverlayPolys feature class (and defined in the Glossary table).

4. Make a stack-unit map in which labels for both the overlying unit (for example, Qal) and the underlying unit (for example, Jm) are shown, separated by a slash. As an example, polygon Qal/Jm is adjacent to polygon Qal/Jk. There are several ways to symbolize the polygons and their bounding contacts, but we won't go into them here (the geologic mapping community as a whole lacks sufficient experience with such maps to prescribe how it should be done). See the next FAQ for suggestions as to how to encode a stack-unit map.

How do I encode a stack-unit map? That is, I'd like to represent bedrock and overlying surficial geology in the same GeMS database. How might I do this?

There are several ways this could be done:

1. MapUnitOverlayPolys. The GeologicMap feature dataset contains a continuous representation of bedrock geology (that is, all areas are mapped). Within the GeologicMap feature dataset, surficial units are represented as a potentially discontinuous set of polygons within a MapUnitOverlayPolys feature class.

Advantages.-This is a simple solution in GeMS and is easily implemented.

Disadvantages.-There is no place to store information about the contacts that separate units within

MapUnitOverlayPolys. Current GeMS tools do not examine topology of MapUnitOverlayPolys. It may not be obvious to the casual database user that MapUnitOverlayPolys contains a representation of surficial geology.

2. Stack-unit map. Kempton (1981) and Berg and Kempton (1988) described a protocol for multilayer maps of near-surface materials. These stack-unit maps are an elegant way to represent our knowledge of multilayered surficial geology while not requiring complete specification of three-dimensional (3D) geometry. Essential aspects of a stack-unit map include the following:

- A stack-unit map is logically a one-layer map

- A stack-unit map describes geology down to a prescribed, commonly shallow, depth (for example, $15 \mathrm{~m}$ )

- Each stack-unit polygon is characterized by a stack of concatenated standard map units-for example, stack-unit Qp/Qvt/Qva indicates peat (Qp) overlying Vashon Till (Qvt) overlying Vashon-age advance-outwash deposits (Qva). The individual map units are termed sublayers. To capture this, the MapUnit field in MapUnitPolys must be replaced with a StackUnit field

- If a sublayer is patchy (or its existence is uncertain), surround the sublayer unit label with parentheses - for example, stack-unit Qp/(Qvt)/Qva indicates that Vashon Till (Qvt) distribution is patchy

- To indicate sublayer thickness, append a number to the sublayer unit symbol—for example, stack-unit Qp2/Qvt3/Qva indicates that $2 \mathrm{~m}$ of peat (Qp) overlies $3 \mathrm{~m}$ of Vashon Till (Qvt)

- The stack-unit protocol, including the depth of depiction, use of parentheses, and thickness measures, should be described in the database-level metadata 
- Some surface contacts will be scratch boundaries where the topmost unit is the same on both sides but a lower layer pinches out

- Implementing a stack-unit map in a GeMS-like database requires a nonspatial junction table that ties the StackUnit field to the MapUnit field in the DescriptionOfMapUnits table (see figure 4-1)

Advantages. - This stack-unit protocol is well adapted to the limitations of much subsurface information.

Disadvantages. - Requires the creation of a new nonspatial table (StackUnitsToMapUnits) and modification of the MapUnitPolys feature class. Substitution of the StackUnit field for the MapUnit field in the MapUnitPolys feature class leads to a database that adheres closely to the spirit of the GeMS schema but is not strictly GeMS compliant.

3. Surficial and bedrock attributes for each polygon. Add BedrockMapUnit and SurficialMapUnit fields to the MapUnitPolys feature class. Populate as appropriate; for each polygon, either the BedrockMapUnit value or the SurficialMapUnit value will be equal to the MapUnit value. To produce a surficial map, access (by coloring or querying on) the SurficialMapUnit field; to get a bedrock map, access the BedrockMapUnit field; or to get a generalized geologic map that incorporates some bedrock and some surficial geology, access the MapUnit field. The Geologic Map of Alaska (Wilson and others, 2015) uses a schema similar to this.

Advantages. - Can be GeMS compliant.

Disadvantages.-Requires processing to calculate which polygon-bounding contacts should be drawn and how they should be drawn - for example, on a surficial map, as dotted contacts for polygons that have identical surficial units but different bedrock units, or, on a bedrock map, as scratch boundaries for polygons that have different surficial units over the same bedrock unit. Such processing can be automated, and the results could be stored as additional attributes of the ContactsAndFaults feature class. Such extensions to the GeMS schema should be clearly documented in associated database-level metadata.

4. Multipatch map. "Multipatch" is the Esri term for a GIS object that consists of a collection of planar patches that represent the boundary of a 3D solid. Like polygons, lines, and points, multipatches are stored in a feature class and may have nonspatial attributes. In a 3D (multilayer) geologic map, constituent multipatches should be adjoining, without gaps or overlaps.

Advantages.-A GeMS-compliant 3D database can be created simply by making MapUnitPolys with the geometry type "MULTIPATCH". This schema allows for complete representation of subsurface-unit geometry.

Disadvantages. - Esri software is currently unable to enforce appropriate topology for a multipatch feature class or to easily create and edit multipatch features. If a multipatch schema is desirable, geometries will likely be generated within other software and then translated into an Esri database. Implementing a 3D analog of ContactsAndFaults requires a geometry type that is not currently supported by Esri software. Specification of a multipatch geologic map requires a density of subsurface information that is rarely available outside of the mineral and hydrocarbon exploitation realms.

5. Multidataset map. Each layer in a multilayer map (for example, a surficial layer, a stratified-bedrock layer, and a crystalline-basement layer) is essentially a separate geologic map and, thus, is stored in a separate feature dataset. To preserve GeMS compliance, one of these feature datasets should be named GeologicMap. Others could be named, for example, SurficialGeologicMap or BasementMap. Each feature dataset contains (at a minimum) feature classes xxxMapUnitPolys and xxxContactsAndFaults, where $\mathrm{xxx}$ is a prefix that identifies the enclosing feature dataset (for example, Layer1). By convention, each feature dataset (layer) has the same map extent. Where material of a layer is not present, set MapUnit = "not present". A contact between a "not present" polygon and other map units is a layer pinch-out line, where the layer above (if present) and the layer below come in contact. Some polygons may have MapUnit = "unmapped area". All layers share DataSources, DescriptionOfMapUnits, GeoMaterialDict, Glossary, and MiscellaneousMapInformation tables. Optionally, the database may contain an elevation surface (raster, TIN, or contours) for the top of each layer. If Layer1 MapUnit = "not present", the elevations of Layer1 and Layer2 are the same. Layer order, elevation-surface names, and layer descriptions are recorded in the nonspatial table LayerList (see table 4-1).

Advantages.-Builds on single-layer GeMS database and honors GeMS' expectations for internal metadata. Existing GeMS tools should work with few or no modifications. The schema looks like the real world (in some regions) where deposits of different geologic episodes are separated by significant unconformities.

Disadvantages. - Does not describe map-unit boundaries within a layer but, rather, only at the top of a layer (though this may be a strength). Inclusion of layer-top-elevation raster data may create unacceptably large databases. 
$A$

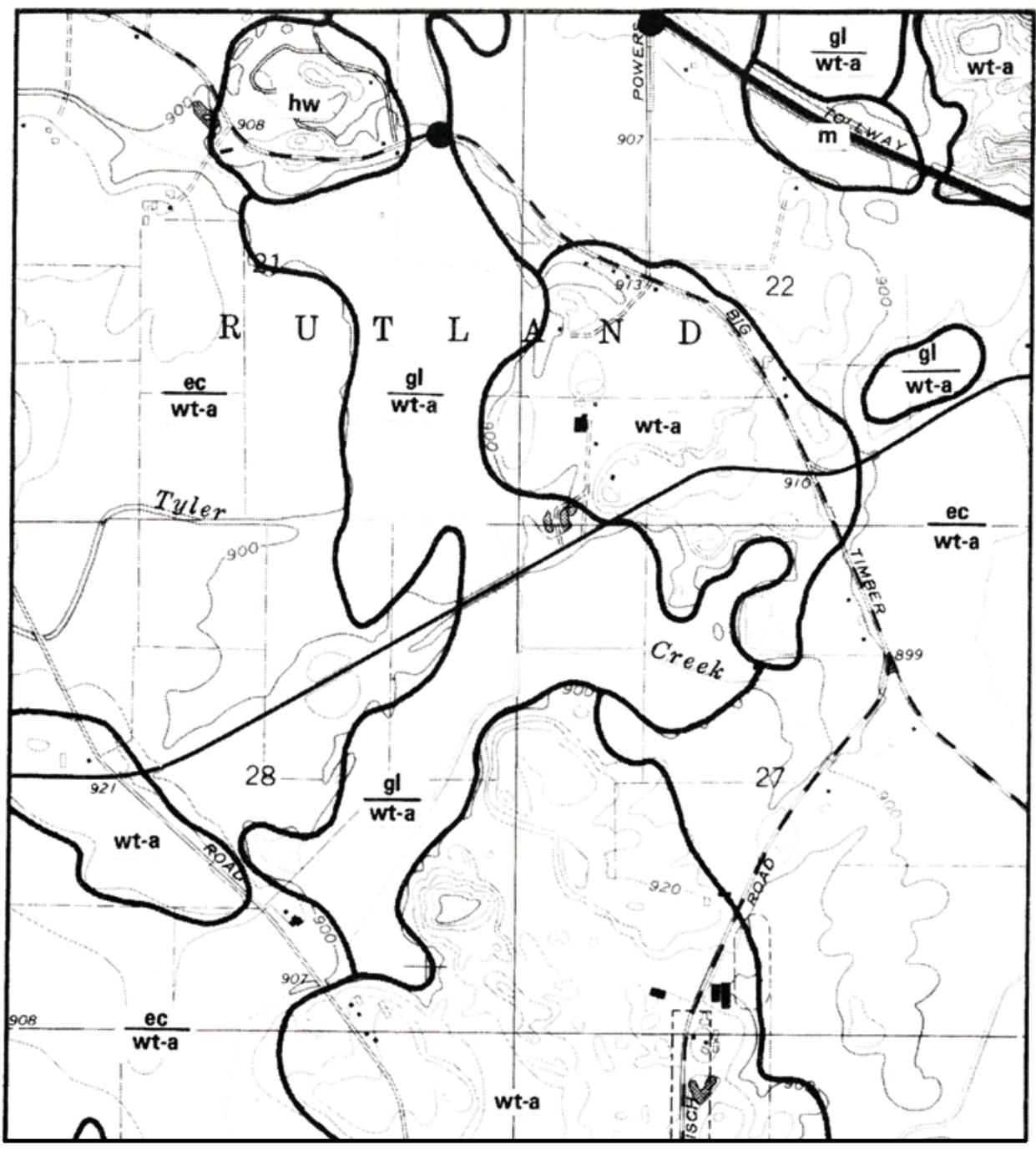

\begin{tabular}{|c|c|c|c|}
\hline B & StackUnit & IdentityConfidence & Notes \\
\hline \multirow{3}{*}{ MapUnitPolys } & hw & certain & <null $>$ \\
\hline & ec/wt-a & certain & $<$ null $>$ \\
\hline & gl/wt-a & certain & $<$ null $>$ \\
\hline
\end{tabular}
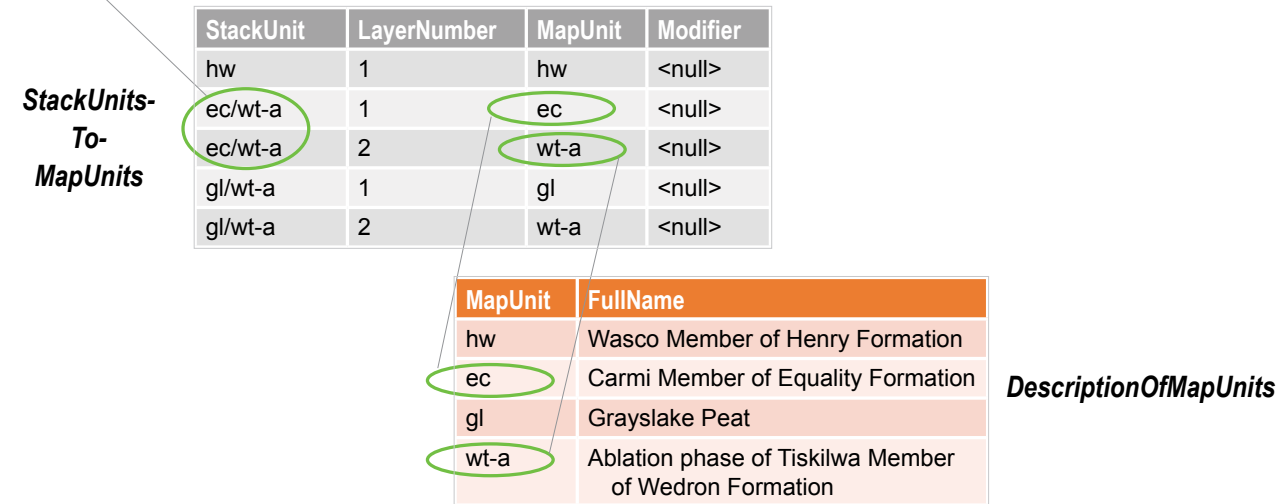

Figure 4-1. GeMS implementation of a stack-unit map. A, Part of a map that shows geologic materials to a depth of $20 \mathrm{ft}$, in the Pingree Grove 7.5-minute quadrangle, Illinois (Kempton, 1981, his fig. 6). B, Corresponding parts of the GeMS tables MapUnitPolys, StackUnitsToMapUnits, and DescriptionOfMapUnits, showing relations that tie values of StackUnit in feature class MapUnitPolys to their constituent MapUnit entries in the nonspatial table DescriptionOfMapUnits. Note that, in StackUnitsToMapUnits, all values of Modifier are <null>; in other locations on this map, values are, for example, "patchy" or "3 ft thick". 
Table 4-1. Examples of selected fields (and their values) in LayerList (a nonspatial table), which organizes and describes datasets within a multidataset map.

\begin{tabular}{lllll}
\hline Order from top & Feature dataset name & \multicolumn{1}{c}{ ElevationObject } & \multicolumn{1}{c}{ Description } & LayerList_ID \\
\hline 1 & SurficialMap & QuadXX_LidarDEM & Unconsolidated-deposit geology at Earth surface & LL1 \\
2 & GeologicMap & $<$ null $>$ & Bedrock geology & LL2 \\
3 & UpperMantleMap & MohoContours & Tomographically guesstimated upper mantle geology & LL3 \\
\hline
\end{tabular}

Such multilayer maps do not replace stack-unit maps with their useful ambiguity about thicknesses and extents of subsurface materials. Nor do they replace multipatch-like maps with their explicit depiction of 3D contacts and map-unit volumes. The USGSAASG-DMT geologic mapping community does not have sufficient experience with these (and other) options to recommend a particular choice. In general, when deciding amongst options, the mapmaker should consider several questions:

1. Are sufficient data available to populate the chosen schema? Traditional two-dimensional geologic maps are descriptions of a fundamentally three-dimensional world, using a set of conventions (map units on the surface, ornamented line symbols that portray the geometry of line features, and explicit or implicit information about the dip of strata) that capitalizes on known surface outcrops and requires few, if any, statements about unobserved subsurface phenomena. Geologists have always desired an explicit 3D representation of the world, but in many instances there are not sufficient data to warrant publishing one.

2. Are naming conventions and documentation clear enough that others will be able to use the dataset? A GeMS map benefits from the availability of GeMS documentation. Extensions to the schema must have names that make their purpose obvious and be well documented in associated metadata.

3. Is it desirable to symbolize the map with surficial geology draped over (and, therefore, obscuring) bedrock geology? The ease of collapsing layers and defining appropriate contact symbolization varies significantly among these schemas.

4. Is the layer-cake approximation intrinsic to multilayer schemas appropriate? For much of North America it is, as a profound regional unconformity separates surficial deposits from significantly older sedimentary and volcanic rocks, which themselves unconformably overlie crystalline bedrock. But, for some areas of the continent, it is not.

How do I encode a three-dimensional (3D) geologic map?

ArcGIS, along with most other GIS software, is not designed to handle 3D (volume) data. See the preceding question for approximations that may be useful. In the future, consideration will be given to more fully adapting GeMS for 3D information.

The Notes field is empty for all records in my ContactsAndFaults feature class. May I delete this field?

Yes, the Notes field is always optional and may be deleted if desired.

What about my fault map? It doesn't show geologic map units.

Strictly speaking, a fault map is not a geologic map, so GeMS does not fully apply. Most fault maps, however, are analogous to certain parts of a geologic map, and so this standard can provide useful guidance. For example, faults could be encoded in the GeologicLines feature class and its associated tables.

May I give the map database to users in another format?

Certainly. But also make the GeMS format available.

My report has an auxiliary map that shows the distribution of sedimentary facies in the Miocene. Where does this map fit in this design?

The answer varies; use your judgment. Not all information depicted in an auxiliary map needs to be a separate digital map (in other words, a separate feature dataset), although that is an acceptable approach. Alternatively, the distribution of Miocene sedimentary facies could be (1) encoded in an optional ExtendedAttributes table for the polygons that represent Miocene sedimentary rocks, (2) portrayed as overlay polygons (in the OverlayPolys feature class), or (3) added as a new polygon feature class. If the map is not complex, then an image of the sedimentary-facies map may suffice.

How can I tell if a database is GeMS-compliant?

The script GeMS_ValidateDatabase_Arc10.py or its replacement (available at the GeMS toolkit site on GitHub; see https://github. com/usgs/GeMS_Tools) tests compliance to the GeMS schema. This script checks that (1) the required tables and feature classes are 
present, (2) all tables and feature classes (both the required ones and any others defined in this schema) have the required fields and field characteristics, (3) non-null values are present in all the fields in which they are required, (4) all tables and feature classes have an $x x x$ ID field (a user-controlled primary key), (5) the values in the $x x x$ ID field (primary keys) are unique within the database, (6) all values in the $x x x$ SourceID field have corresponding entries in the DataSources table, (7) no unreferenced entries are in the DataSources table, (8) all values in the Type, IdentityConfidence, and ExistenceConfidence fields have corresponding entries in the Glossary table, (9) no unreferenced entries are included in the Glossary table, (10) no false null values (that is, no empty or whitespace strings) are present, (11) values of the MapUnit field in the MapUnitPolys feature class (and in the CMUMapUnitPolys and any CSxMapUnitPolys [for cross sections] feature classes, if present) correspond to values in the DescriptionOfMapUnits table, and (12) values of the HierarchyKey field in the DescriptionOfMapUnits table are well structured.

The script GeMS_TopologyCheck_Arc10.py (also available at the GeMS toolkit site on GitHub) or its replacement provides additional checks for both automation errors and (potentially) geologic blunders. This script builds and evaluates an ArcGIS topology using the following rules: (1) no overlaps, self-overlaps, or self-intersections are in the ContactsAndFaults feature class, (2) no gaps or overlaps are in the MapUnitPolys feature class, and (3) all boundaries in the MapUnitPolys feature class are covered by (that is, exactly coincident with) lines in the ContactsAndFaults feature class. The script also evaluates node topology, summarizes the map units that bound lines in the ContactsAndFaults feature class, and checks for duplicate point features. Note that results of the GeMS_TopologyCheck_Arc10.py script should be taken with a grain of salt, as some issues flagged by this script may not be errors.

\section{What about annotation?}

There are multiple ways to create and store annotation. We are not sure what data structure will best facilitate publication-quality cartography and allow for the economical creation and editing of annotation (and leaders), and so we have not prescribed a protocol for annotation. Map authors may wish to create one or more Esri annotation feature classes to store their annotation, along with instructions on how to use them.

How do I use a GeMS-compliant database to make a publication-quality map graphic?

The answer to this question is not trivial, but by standardizing the database design, we hope to see the emergence of tools from the community to provide answers. The following are some suggestions:

1. Use high-quality symbology such as that contained in the ArcGIS .style file FGDC_GSC_20100414.style, prepared by the Geological Survey of Canada (see discussion in the "Symbolization" section, in the main document above).

2. Create visually pleasing symbolization of highly segmented faults that have complex line ornamentations (for example, sawteeth on thrust faults) by making continuous, throughgoing fault traces (known as metafaults in GIS jargon, these are analogous to routes in Workstation ArcInfo), in order to reduce or eliminate overcrowding of line ornamentations. To do this, draw individual fault segments using thick (fault-weight) solid, dashed, or dotted line symbols, as appropriate, without the sawteeth; merge these line segments into metafaults and then smooth (generalize, spline) them; then draw the smoothed metafaults using the sawteeth only, without the line stroke.

3. Create effective annotation (see previous FAQ for discussion on annotation). Map-unit symbols, dip and plunge values for measured orientations, text associated with line features or other types of point data, and place names all may need to be repositioned, duplicated, or eliminated to create a legible map graphic. Some map-unit labels may need to have leaders added. Note that at this time we are not aware of any tools that can successfully automate this task.

4. To the greatest extent possible, prepare as much of the map (inside the neatline) as is possible in ArcMap. Avoid cartographic work inside the neatline using desktop publishing software such as Adobe Illustrator, as doing so increases the likelihood of synchronization problems between the geology portrayed on the map and that which is recorded in the database.

5. Prepare the layout of the map sheet (outside the neatline) using desktop publishing software, as text formatting and figure placement are much easier than doing so in ArcMap.

I don't know what metadata for a geologic map should look like. What should I do?

Please refer to USGS's website on formal, FGDC-compliant metadata (see https://geology.usgs.gov/tools/metadata/).

Who is going to enforce the use of GeMS?

When GeMS is fully adopted by the National Cooperative Geologic Mapping Program, conformance will be required upon delivery of products funded by the Program. If widely adopted, users will demand conformance so that tools developed to manipulate the databases work properly. 


\section{References Cited in Appendix 4}

Berg, R.C., and Kempton, J.P., 1988, Stack-unit mapping of geologic materials in Illinois to a depth of 15 meters: Illinois State Geological Survey Circular 542, 23 p., 4 plates, https://isgs.illinois.edu/publications/c542.

Kempton, J.P., 1981, Three-dimensional geologic mapping for environmental studies in Illinois: Illinois State Geological Survey, Environmental geology note 100, 43 p., https://archive.org/details/threedimensionall00kemp.

Wilson, R.H., Hults, C.P., Mull, C.G., and Karl, S.M., compilers, 2015, Geologic map of Alaska: U.S. Geological Survey Scientific Investigations Map 3340, 197 p., 2 sheets, scale 1:584,000, https://doi.org/10.3133/sim3340. 


\section{Appendix 5. Changes from NCGMP09 v.1.1 to GeMS}

[Names of sections in this document have been highlighted in bold type. Substantive changes to the GeMS schema from NCGMP09 v.1.1 (U.S. Geological Survey National Cooperative Geologic Mapping Program, 2010) are indicated by magenta type. Other changes listed are modifications made to this documentation since NCGMP09 v.1.1]

Throughout document -

Changed name of schema from NCGMP09 to GeMS

Changed title of documentation to "GeMS (Geologic Map Schema)—A Standard Format for the Digital Publication of Geologic Maps"

Modified authorship statement

Added Table of Contents to front matter of document

Made revisions for clarity

Added several footnotes to help clarify terminology

Changed most instances of "geodatabase" to "database"

Reduced use of first-person voice

Recast diagrams of fields (and their values) into numbered tables that can be easily located using the Table of

Contents

For all numbered tables (as applicable):

Added "Field name", "Description", and "Notes on usage" column headings to improve structure and organization Made significant revisions for clarity, consistency, and completeness

Highlighted (using magenta bold type) the contents of fields that must be defined in Glossary table where applicable and explained this red bold type in bracketed headnotes

Moved descriptions of Type (or similar) fields to top of tables and moved descriptions of TableName_ID fields to bottom. Although field order is not prescribed in GeMS, a consistent field order makes the schema easier to comprehend; in addition, ArcGIS workflow considerations make it desirable to have the Type field (or similar) first

Removed RuleID and Override fields and values (inclusion of these fields in GeMS is perceived by the USGS-AASG-DMT community as an impediment to understanding the schema by those who do not use Esri's cartographic representations; furthermore, those who use cartographic representations can always add these optional fields)

Stated that Notes fields are always optional

Changed "Nulls OK" to "Null values permitted"

Renumbered appendixes from A-E to 1-5

In Introduction section -

Changed intent from "proposes a schema" to "describes and defines a schema"

Changed "two decades" to "three decades"

Added evolution of the schema after NCGMP09 v.1.1

Deleted former Contents and Review, Comment and Revision sections

Moved Acknowledgments section to front matter, before Table of Contents

In Design Considerations section -

Moved Content of a Traditional Geologic Map and following sections into new Content of a Geologic Map section (see below)

In Content of a Geologic Map section -

Highlighted (using MAGENTA ALL CAPS bold type) fundamental map content within GeMS that is further discussed in sections that follow, where the GeMS schema is described in detail

Highlighted (using BLACK ALL CAPS bold type) secondary and supporting map content whose digital form is prescribed herein 
Highlighted (using italic bold type) secondary and supporting map content for which a digital form is not specified herein Made minor revisions to Symbolization discussion for clarity, consistency, and completeness

In Extensions to Traditional Geologic Map Content section:

Made significant revisions to Glossary of Terms section (renamed from Glossary)

Made significant revisions to Classification of Geologic Materials (renamed from General Lithology)

In Feature-Level Metadata section:

Significantly rewrote Locational Confidence (Spatial Accuracy) section; added new example picklist (table 1) for LocationConfidenceMeters values (goal is to better explain intent of

LocationConfidenceMeters attribute and better illustrate its use)

Added new example picklist (table 2) for IdentityConfidence values to Scientific Confidence,

Identity Confidence, and Existence Confidence section

Added new Working with Multiple Feature Attributes section

In Naming Database Elements section:

Changed CamelCase to the more accurate PascalCase

Reordered bullets to separate cognitive concerns from coding concerns

Revised Transparent Identifiers section for clarity

In File Formats section (renamed from Open File Formats):

Specified use of .gdb only (not .mdb)

Substantially revised discussion of text, table, and image formats for clarity

In Required, As-Needed, and Optional Elements of a Digital Geologic Map Publication section (renamed from Required,

As-Needed, and Optional Contents of a Digital Geologic Map Publication) -

Removed mentions of mapXYZ-mdb.zip, base.mdb, in response to suggestions to deprecate .mdb format

Renamed base.gdb to mapXYZ-base.gdb

Recast list of required, as-needed, and optional map elements into tables 3, 4, and 5 for clarity

In The Database Design section (renamed from The Geodatabase Design) -

Noted, in initial paragraph, that additional optional elements are permitted by schema

In General Considerations section:

Renumbered figures $2 \mathrm{~A}, 2 \mathrm{~B}$, and $2 \mathrm{C}$ to figures 2,3 , and 4 , respectively

Added new Field Hygiene section:

Clarified that schema does not, in general, prescribe field lengths

Stated that required fields should not be deleted and that repurposing of required fields is prohibited

Described use of explicit null values

Stated that Notes fields are optional and may be omitted

Moved paragraphs 2, 3, and 4 of The Database Design section (originally named The Geodatabase Design) into Field Hygiene section

Added four new tables illustrating use of primary keys (TableName_ID field) and foreign keys TableNameID field)

In Type, MapUnit, Label, and Symbol Fields section (renamed from Type, Label, and Symbol Fields):

Added separate discussion of MapUnit field

Expanded discussion of Label field and how Label field differs from MapUnit field, especially when used with FGDCGeoAge font

Added new table 10 showing examples of values in Type, Symbol, and Label fields in a point feature class and resulting map images

Added new table 11 showing examples of values in MapUnit, IdentityConfidence, Symbol, and

Label fields in MapUnitPolys feature class and resulting map images

In Directional Lines section:

Expanded discussion of "right-hand rule" 
In Required Elements section:

In GeologicMap (Feature Dataset) section:

In MapUnitPolys (Polygon Feature Class) section:

Added further discussion of using FGDCGeoAge font to produce geologic age symbol characters

Added separate Topology Rules section

In ContactsAndFaults (Line Feature Class) section:

Added separate Topology Rules section

In DescriptionOfMapUnits (Nonspatial Table) section:

Deleted former table 1 and references thereto (material is now covered in appendix 3)

Deleted paragraph suggesting development of more structured DMU descriptions

In table 14:

Revised Name and Description fields to state that headings should be entered in the Name field and associated headnotes, if present, should be entered in the Description field

Added footnotes to Name and FullName fields stating that formal names should be verified in Geolex (see https://ngmdb.usgs.gov/Geolex/)

Added sentence to Name field stating that text in this field should have no font or hierarchy specifications

Rewrote HierarchyKey field description for clarity

Added example showing how ParagraphStyle field works (that is, values are type and paragraph specifications, which are defined in Glossary table)

Replaced GeneralLithology and GeneralLithologyConfidence fields with GeoMaterial and GeoMaterialConfidence fields

Added new Notes on the Content of the Description Field section that contains extensive new material on the content and writing of map-unit descriptions, mostly quoted from USGS's "Suggestions to Authors of the Reports of the United States Geological Survey_Eighth Edition" (U.S. Geological Survey, in press) and R.E. Wells (USGS, written commun., 2010)

Added new Populating the DescriptionOfMapUnits Table section:

Moved discussion of GeoMaterial and GeoMaterialConfidence fields here

Added short paragraph that refers reader to appendix 3, which illustrates construction of

HierarchyKey values

Added new Order and Rank of Map Units section:

Expanded discussion describing use of ParagraphStyle values

In DataSources (Nonspatial Table) section:

Added optional URL field

Deleted sentence at end that refers to ChangeLog

Added new GeoMaterialDict (Nonspatial Table) section (and new table 17)

Moved DataSourcePolys (Polygon Feature Class) section to Examples of Other As-Needed Feature

Classes section (in As-Needed Elements section)

In Glossary (Nonspatial Table) section:

Deleted Qualifier and Property fields from list of fields for which all values must be defined in Glossary table (fields are within ExtendedAttributes table, which has been removed from this version)

Deleted GeoMaterial field from list of fields whose values must be defined in Glossary, as these values are now defined in GeoMaterialDict table (see appendix 1)

Deleted sentence "Lithology terms used in GeneralLithology must not be redefined from the NGMDB standard" from discussion (field name has changed, and topic is addressed in GeoMaterialDict section) 
In As-Needed Elements section:

Clarified that, although GeMS does not prescribe the use of any particular as-needed feature classes, any feature class described here that is used by a GeMS database should conform to these descriptions

In Guidelines for Naming and Designing Additional Feature Classes section (renamed from Guidelines for Naming and Designing Additional Polygon, Line, and Point Feature Classes):

Changed "CamelCase" to the more accurate term "PascalCase"

Deleted last sentence requesting comments on naming of feature classes

In Structure of Point Data section:

In Point Feature Classes in General section:

Added short paragraph explaining utility of MapUnit field

Changed "Sample-oriented point feature classes shall have the fields" to "We also recommend that sample-oriented point feature classes include certain additional fields"

Renamed StationID field to StationsID field

Deleted MapX and MapY fields, as they are redundant in a feature class

Split former Some Examples of As-Needed Feature Classes section into two new sections (Examples of As-Needed Point Feature Classes and Examples of Other As-Needed Feature Classes)

In new Examples of As-Needed Point Feature Classes section:

In OrientationPoints (Point Feature Class) section:

Deleted last sentence that referred to ExtendedAttributes table

In table 22:

Added StationsID and MapUnit fields, for consistency with Point Feature

Classes in General section

Renamed DataSourceID field to LocationSourceID field

Added OrientationSourceID field

In GeochronPoints (Point Feature Class) section:

In table 23:

Renamed StationID field to StationsID field

Renamed DataSourceID field to LocationSourceID field

Added AnalysisSourceID field

Added ErrorMeasure field

In Stations (Point Feature Class) section:

Added short paragraph explaining utility of ObservedMapUnit field

In table 24

Changed "Null values not permitted" for MapUnit field to "Null values permitted only for points outside the extent of MapUnitPolys feature class"

Added "suggest setting default value $=0$ ( $=$ display at all scales)" for PlotAtScale field In table 25:

Added "and affiliation" for Observer field

Added new MapUnitPoints (Point Feature Class) section

In new Examples of Other As-Needed Feature Classes section:

Added new MapUnitLines (Line Feature Class) section

In IsoValueLines (Line Feature Class) section:

In table 28:

Added ValueConfidence field 
In Overlay Polygons (the MapUnitOverlayPolys and OverlayPolys Polygon Feature Classes) section (renamed from OtherPolys):

Added discussion of MapUnitOverlayPolys feature class

Renamed OtherPolys feature class to OverlayPolys feature class

Added separate Topology Rules section

Explanatory text revised significantly

In DataSourcePolys (Polygon Feature Class) section:

Changed from a required element to an as-needed element

Made minor revisions to introductory paragraph

Added separate Topology Rules section

Deleted topology rule "Polygon boundaries may in part be coincident" (deemed unnecessary)

In Symbolization section -

Made substantial revisions for clarity

Added new URL for Esri’s Geologic Mapping Template

Changed "we also require provision of an ArcReader document" to "we also suggest that an ArcReader document

(.pmf file) be provided" (that is, changed inclusion of ArcReader document from required to optional)

In Shapefile Version of the Database section (renamed from Shapefile Versions of the Database) -

Dropped requirement for "simple" shapefile version and all references to its construction

Deleted Simple Version section

Deleted Open Version section

In Appendix 1. Terms and Definitions for the GeoMaterial and GeoMaterialConfidence Fields (renamed from Appendix A. Lithology and Confidence Terms for GeneralLithology) -

Made minor changes for clarity

Removed reference to future evolution of term list

Added new Scope and Intent section

In GeoMaterial Terms section (renamed from GeneralLithology):

Under term Sedimentary rock, added term Chert

Under term Carbonate rock, added terms Limestone and Dolomite

Under term Extrusive igneous material, added term Volcanic mass flow

Under term Regional metamorphic rock, of unspecified origin, added term Lower-grade metamorphic rock, of unspecified origin

Under term Metasedimentary rock:

Added term Meta-carbonate rock

Deleted term Marble

Under term Metaigneous rock:

Added term Meta-ultramafic rock

Added term Meta-mafic rock

Added term Meta-felsic and intermediate rock

Added term Meta-volcaniclastic rock

Numerous minor changes in term descriptions, for clarity and consistency

Moved Soller (2009) reference into new Reference Cited in Appendix 1 section

Placed references to sources of GeoMaterial terms into new Published and Unpublished Sources for Terms and

Definitions (Modified as Needed) in the GeoMaterial Field, as Listed in Appendix 1 section 
In Appendix 2. Optional Database Elements (renamed from Appendix B. Optional Elements) -

In Correlation of Map Units (Feature Dataset) section:

Deleted second paragraph

Added discussion of CMUMapUnitLines and CMUMapUnitPoints feature classes

In CMUMapUnitPolys (Polygon Feature Class) section:

Expanded discussion of ghost boxes

Deleted CMUPoints (Point Feature Class) section

Added new MiscellaneousMapInformation (Nonspatial Table) section because new MiscellaneousMapInformation table has been added

Deleted ExtendedAttributes and GeologicEvents sections because ExtendedAttributes and GeologicEvents tables have been deleted (to our knowledge, no one in the USGS-AASG-DMT community has implemented either the ExtendedAttributes or the GeologicEvents tables in a published database; however, this does not preclude the implementation of either table)

Added new Deprecated Nonspatial Tables section that refers those interested to NCGMP09 v.1.1 documentation for descriptions of ExtendedAttributes and GeologicEvents tables

Added reference for NCGMP09 v.1.1 and put it in new Reference Cited in Appendix 2 section

Deleted former Appendix C. Building a Compliant Database because this material, in revised form, appears in the wiki associated with the GeMS toolkit (see https://github.com/usgs/GeMS_Tools)

Added new Appendix 3. Parsing a Description of Map Units (DMU) into the DescriptionOfMapUnits Table and the HierarchyKey Field -

In new The DescriptionOfMapUnits Table section, added explanation of, and examples that illustrate, how to translate the contents of a Description Of Map Units (DMU) into the DescriptionOfMapUnits table

In new The HierarchyKey Field section, added description of, and examples that illustrate, the HierarchyKey field, giving rules for its construction

Placed references in new References Cited in Appendix 3 section

In Appendix 4. Frequently Asked Questions (FAQs) -

Added new question How do I get started?

For the question How do I represent dikes?, changed "OtherLines" in the second choice to "GeologicLines" ("OtherLines" was an error in v.1.1)

Added new questions How do I represent other small bodies of rock?, What about bedrock contacts under alluvium?, and How do I encode a stack-unit map? That is, I'd like to represent bedrock and overlying surficial geology in the same GeMS database. How might I do this?

For the question The Notes field is empty for all records in my ContactsAndFaults feature class. May I delete this field?, changed answer to indicate that Notes fields are optional and may be deleted

For the question What about my fault map? It doesn't show geologic units, changed answer to suggest the encoding of faults in GeologicLines rather than ContactsAndFaults

For the question My report has an auxiliary map that shows the distribution of sedimentary facies in the Miocene.

Where does this map fit in this design?, changed "feature class" in second sentence to "feature dataset" ("feature class" was an error in v.1.1)

For the question How can I tell if a database is GeMS-compliant?, substantially expanded answer

For the question How do I use a GeMS-compliant database to make a publication-quality map graphic?, made major revisions for clarity and completeness

Deleted question I've got a better design for a standard geologic-map database. How do I go about getting this proposal changed?

Placed references in new References Cited in Appendix 4 section

Added new Appendix 5. Changes from NCGMP09 v.1.1 to GeMS 


\section{References Cited in Appendix 5}

U.S. Geological Survey, in press, Suggestions to Authors of the Reports of the United States Geological Survey, Eighth edition: U.S. Geological Survey website.

U.S. Geological Survey National Cooperative Geologic Mapping Program, 2010, NCGMP09—Draft standard format for digital publication of geologic maps, version 1.1, in Soller, D.R., ed., Digital Mapping Techniques '09-Workshop Proceedings: U.S. Geological Survey Open-File Report 2010-1335, p. 93-146, 4 appendixes, https://pubs.usgs.gov/of/2010/1335/pdf/ usgs_of2010-1335_NCGMP09.pdf. 
Menlo Park Publishing Service Center, California

Manuscript approved for publication March 10, 2020

Edited by Taryn A. Lindquist

Layout by Cory Hurd 


\section{$\frac{\mathbb{3}}{3}$}

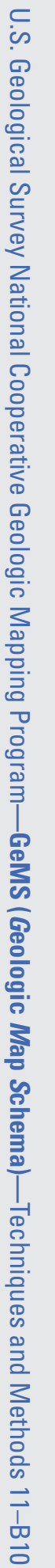

